\title{
Recent advances in directional statistics
}

\author{
Arthur Pewsey ${ }^{1,3}$ and Eduardo García-Portugués ${ }^{2}$
}

\begin{abstract}
Mainstream statistical methodology is generally applicable to data observed in Euclidean space. There are, however, numerous contexts of considerable scientific interest in which the natural supports for the data under consideration are Riemannian manifolds like the unit circle, torus, sphere and their extensions. Typically, such data can be represented using one or more directions, and directional statistics is the branch of statistics that deals with their analysis. In this paper we provide a review of the many recent developments in the field since the publication of Mardia and Jupp (1999), still the most comprehensive text on directional statistics. Many of those developments have been stimulated by interesting applications in fields as diverse as astronomy, medicine, genetics, neurology, aeronautics, acoustics, image analysis, text mining, environmetrics, and machine learning. We begin by considering developments for the exploratory analysis of directional data before progressing to distributional models, general approaches to inference, hypothesis testing, regression, nonparametric curve estimation, methods for dimension reduction, classification and clustering, and the modelling of time series, spatial and spatiotemporal data. An overview of currently available software for analysing directional data is also provided, and potential future developments discussed.
\end{abstract}

Keywords: Classification; Clustering; Dimension reduction; Distributional models; Exploratory data analysis; Hypothesis tests; Nonparametric methods; Regression; Serial dependence; Software; Spatial statistics.

\section{Introduction}

Directional statistics is that branch of statistical methodology specifically designed for use with observations that are directions. A direction observed in the plane $\mathbb{R}^{2}$, like wind direction, can be represented by an angle, $\theta$, typically in $[0,2 \pi)$ or $[-\pi, \pi)$, measured in a specified direction from a specified origin, or by the unit vector $\boldsymbol{x}=(\cos \theta, \sin \theta)^{\prime}$ for which $\|\boldsymbol{x}\|=\sqrt{\boldsymbol{x}^{\prime} \boldsymbol{x}}=1$. The natural support for such directions is the circumference of the unit circle, $\mathbb{S}^{1}$; data on them being referred to as circular. The term "circular data" is also used to distinguish them from data with the real line $\mathbb{R}$ (or some subset of it) as their support, which henceforth we will refer to as linear data. Certain calculations can be performed more efficiently using the complex representation $z=\mathrm{e}^{\mathrm{i} \theta}$, where $\mathrm{i}=\sqrt{-1}$, for which $|z|=1$ and $\operatorname{Arg}(z)=\theta \in[-\pi, \pi)$. Closely related to circular data are axial data, which arise when axes, for which the angles $\theta$ and $\theta+\pi$ are indistinguishable, are observed. Observations made on directions in $\mathbb{R}^{3}$, like the positions of stars on the celestial sphere, can be represented by pairs of angles or $3 \times 1$ unit column vectors, have natural support the unit sphere, $\mathbb{S}^{2}$, and are referred to as being spherical. Circular and spherical data are the most commonly occurring forms of directional data. Since their supports are compact manifolds, it is (generally) inappropriate, and can prove thoroughly misleading, to apply standard statistical methods, designed for observations with more familiar supports like $\mathbb{R}^{d}, d \geq 1$, to them.

Other data types that fall within the remit of directional statistics include toroidal and cylindrical data: toroidal data, with support the unit torus, $\mathbb{T}^{2}=\mathbb{S}^{1} \times \mathbb{S}^{1}$, arising when observations on a pair

\footnotetext{
${ }^{1}$ Department of Mathematics, University of Extremadura (Spain).

${ }^{2}$ Department of Statistics, Carlos III University of Madrid (Spain).

${ }^{3}$ Corresponding author. e-mail: apewsey@unex.es.
} 
of circular variables are made, and cylindrical data, with support the cylinder $\mathbb{S}^{1} \times \mathbb{R}$ or some subset of it, when observations are made on a pair consisting of one circular and one linear variable. For example, toroidal data are obtained when wind direction is recorded at two different meteorological stations, and cylindrical data if, instead, wind direction and velocity are jointly measured at the same station.

In applications, data on these various manifolds, or their generalisations, such as the unit $d$ dimensional sphere, $\mathbb{S}^{d}$, and the $d$-torus, $\mathbb{T}^{d}=\left(\mathbb{S}^{1}\right)^{d}, d \geq 1$, might be observed and analysed as regression, time series, spatial or spatio-temporal data. Henceforth, we will use the term "spherical data" to refer to data on any $\mathbb{S}^{d}$ with $d \geq 1$, not just $\mathbb{S}^{2}$, unless specifically mentioned otherwise.

Directional statistics can also be applied to data that are not originally directions but which can be represented on, or transformed to, one of the manifolds referred to previously. For instance, times on the 24 hour clock can be analysed as circular data after transferring them to the unit circle (see, e.g., Gill and Hangartner, 2010). More generally, methods for spherical data can be applied to data originally observed in Euclidean space, $\boldsymbol{x}_{1}, \ldots, \boldsymbol{x}_{n} \in \mathbb{R}^{d+1}$, after their Euclidean normalisation to $\boldsymbol{x}_{1} /\left\|\boldsymbol{x}_{1}\right\|, \ldots, \boldsymbol{x}_{n} /\left\|\boldsymbol{x}_{n}\right\| \in \mathbb{S}^{d}$, a form of transformation often encountered (see, e.g., Banerjee et al., 2005).

Rotation groups, Stiefel and Grassmann manifolds, the elements of which are orthonormal frames and subspaces of $\mathbb{R}^{d}$, respectively, and other sample spaces such as hyperboloids, complex projective spaces, and general manifolds, are also important to the field but here, because of length restrictions, we refer only tangentially to certain developments related to them. Specifically, we do not consider models for rotations in $\mathbb{R}^{3}$ despite the fact that a $3 \times 3$ rotation matrix can be represented as a $4 \times 1$ unit vector called a quaternion, and modelling such rotations is equivalent to modelling axial data on $\mathbb{S}^{4}$. We would direct the reader interested in these topics to Mardia and Jupp (1999, Chapter 13), Mardia and Patrangenaru (2005), Chirikjian and Kyatkin (2001), Chikuse (2012), Arnold and Jupp (2018), and Rivest and Oualkacha (2018). An important related field is shape analysis (Kendall et al., 1999; Dryden and Mardia, 2016), where a preshape corresponding to a configuration of $k$ landmarks in $\mathbb{R}^{d}$ can be regarded as a point on $\mathbb{S}^{d(k-1)-1}$.

The last article-length review of directional statistics was Jupp and Mardia (1989). It contained an extensive bibliography which included virtually all publications on directional statistics between 1975 and 1988. In their review, the authors sought to unify the theory of directional statistics from a mathematical perspective. In attempting to doing so, they referred to five key underpinning ideas: (i) exponential families; (ii) transformation structure; (iii) tangent-normal decomposition; (iv) transformation (of a directional problem) to a multivariate one; (v) the central limit theorem (CLT), and three basic approaches to directional statistics, termed the embedding, wrapping, and intrinsic approaches. All of these underlying principles have been fundamental to the ongoing development of the field, apart perhaps from the first. Whilst exponential models have certain appealing mathematical and inferential properties, insistence on them has largely been abandoned in recent years, primarily because of an increasing awareness of the need to model distributional features beyond location and concentration, such as the varying levels of skewness and peakedness frequently exhibited by real data. Moreover, directional data are often multimodal and finite mixture distributions, which do not belong to the exponential family, are natural choices with which to model them.

Books covering numerous facets of directional statistics published prior to the review of Jupp and Mardia (1989) include Mardia (1972), Batschelet (1981), Watson (1983), Fisher et al. (1987), and Fisher (1993). Those published after that review include Mardia and Jupp (1999), Jammalamadaka and SenGupta (2001), Pewsey et al. (2013), Ley and Verdebout (2017a), and Ley and Verdebout (2018), the latter being an excellent overview of interesting and important modern applications of 
directional statistics. We take as our definition of "recent developments" those that have appeared in the literature since the publication of Mardia and Jupp (1999), still the most comprehensive book-length treatment of the field. Whilst many, but certainly not all, of the themes we discuss are addressed in the books of Ley and Verdebout, our aim has been to provide a concise review of the most important developments since the publication of Mardia and Jupp (1999) which is as exhaustive as possible, subject to length constraints. Given the latter, we have concentrated on describing key ideas and directing the interested reader to relevant original sources where more detailed information can be found. With the increasing pace of advances in the field, it is perhaps inevitable that we will have overlooked some developments. We hope that the number of such omissions is minimal, and apologise in advance for any that might have arisen.

Important areas of application that have stimulated much of the recent research activity in the field include bioinformatics (Boomsma et al., 2008; Mardia et al., 2018), astronomy (Cabella and Marinucci, 2009; Marinucci and Peccati, 2011), medicine (Vuollo et al., 2016; Pardo et al., 2017), genetics (Eisen et al., 1998; Dortet-Bernadet and Wicker, 2008), neurology (Gu et al., 2004; Kaufman et al., 2005), aeronautics (Horwood and Poore, 2014), acoustics (McMillan et al., 2013; Traa and Smaragdis, 2013), image analysis (Jung et al., 2011; Esteves et al., 2020), text mining (Dhillon and Modha, 2001; Banerjee et al., 2005), machine learning (Hamsici and Martinez, 2007; Sra, 2018), and the modelling of wildfires (García-Portugués et al., 2014; Ameijeiras-Alonso et al., 2018) and sea conditions (Jona-Lasinio et al., 2012, 2018; Lagona, 2018).

The remainder of the paper is structured as follows. In Section 2 we review advances in exploratory data analysis before proceeding to distributional models in Section 3, general approaches to inference in Section 4, and to hypothesis testing in Section 5. Section 6 discusses developments for correlation and regression. Section 7 focuses on advances in nonparametric curve estimation, Section 8 on methods for dimension reduction, and Section 9 on classification and clustering. Developments in modelling serial dependence, and spatial and spatio-temporal data, are reviewed in Sections 10 and 11, respectively. Advances in data depth, the design and analysis of experiments, order-restricted analysis, outlier detection, and compositional data analysis are considered more briefly in Section 12. An overview of the software currently available for analysing directional data is provided in Section 13. The paper ends with the brief Section 14 in which conclusions are drawn and potential future developments discussed.

\section{Exploratory data analysis}

As for other types of data, the exploratory analysis of directional data usually begins with an inspection of some graphical summary of the data. Various adaptations of the popular rose diagram have been developed recently. Munro and Blenkinsop (2012) introduced a moving rose diagram and applied it to circular datasets from the Earth sciences. Rodgers et al. (2014) proposed the wrap-around time series plot for displaying time series exhibiting periodic patterns. Morphet and Symanzik (2010) proposed the circular dataimage, a graphical tool that uses a colour wheel to encode directions over a map. Rose diagrams, circular histograms, and other circular plots were adapted in $\mathrm{Xu}$ and Wang (2020) so as to obtain area-proportional displays.

Circular boxplots have been investigated only relatively recently (but see Anderson (1993)). For $\theta_{1}, \ldots, \theta_{n} \in[0,2 \pi)$, Abuzaid et al. (2012) advocated one centred on the circular median, $M=$ $\arg \min _{\phi \in[0,2 \pi)} \sum_{i=1}^{n} d_{c}\left(\phi, \theta_{i}\right)$, where

$$
d_{c}(\phi, \theta)=\pi-|\pi-| \theta-\phi||
$$

is the shortest arc length distance between the two angles $\phi, \theta \in[0,2 \pi)$ when represented as points on the circumference of the unit circle. In an attempt to mimic more closely Tukey's original con- 
struction, Buttarazzi et al. (2018) proposed a depth-based boxplot in which the observations are ranked from the antimedian to the median. For both proposals, the fences are calibrated assuming an underlying von Mises distribution (see Section 3.1).

The SiZer, an abbreviation for "significant zero crossings of derivatives" (Chaudhuri and Marron, 1999), is a handy tool used to identify statistically significant features at different scales, such as modes and antimodes, in univariate linear data. A circular adaptation of the SiZer, the CircSiZer, based on the kernel density estimator (18) and bootstrap confidence intervals to assess the significance of smoothed derivatives, was proposed in Oliveira et al. (2014). It can also be employed to explore significant features in linear-circular regression. In both contexts, smoothing is based on a von Mises kernel with concentration parameter $\kappa$ (see (18) and (20)). This kernel was shown not to be "causal" by Huckemann et al. (2016), in the sense that its convolution with a circular density function is not guaranteed to maintain or reduce the number of modes as the level of smoothing, $1 / \kappa$, increases. They proved that, among all the circular kernels satisfying certain mild assumptions, the wrapped normal (see Section 3.1) is the only one that yields circular causality. Employing such a kernel, they proposed the Wrapped SiZer (WiZer), with asymptotic confidence intervals used to assess the significance of smoothed derivatives. Extension of the SiZer methodology to spherical data led to the SphereSiZer of Vuollo and Holmstrom (2018), itself inspired by the adaptation of the SiZer to bivariate linear data by Godtliebsen et al. (2002). The SphereSizer uses a von Mises-Fisher kernel density estimator for data on $\mathbb{S}^{2}$ (see (17)), and bootstrap confidence intervals to assess the significance of smoothed gradients. It produces a movie, indexed by the smoothing scale, that displays statistically significant density gradients as a vector field and highlights spherical regions with high density.

\section{Distributional models}

\subsection{Circular models}

The probability density function (pdf) of an absolutely continuous circular random variable (rv) $\Theta$, $f_{\Theta}$, is such that $f_{\Theta}(\theta) \geq 0$ and $f_{\Theta}(\theta+2 \pi)=f_{\Theta}(\theta)$ for almost all $\theta \in \mathbb{R}$. Also, $\int_{\theta}^{\theta+2 \pi} f_{\Theta}(\omega) \mathrm{d} \omega=1$. Thus, $f_{\Theta}$ is non-negative, $2 \pi$-periodic, and integrates to 1 over any interval of length $2 \pi$. As a consequence of this latter property, it is usual to define a circular pdf through its values on $[0,2 \pi)$ or $[-\pi, \pi)$. For instance, the circular uniform distribution, the most fundamental model for circular data corresponding to there being no preferred direction, has pdf $f_{\Theta}(\theta)=1 /(2 \pi), \theta \in[0,2 \pi)$. The circular cumulative distribution function (cdf) is defined as the non-periodic function $F_{\Theta}(\theta)=\int_{\theta_{0}}^{\theta} f(\omega) \mathrm{d} \omega$, with $\theta_{0}$ typically being 0 or $-\pi$.

Six general approaches have often been used to generate models for circular data (Mardia and Jupp, 1999, Section 3.5): wrapping, projection, perturbation, conditioning, diffusion, and characterisations such as maximum likelihood or maximum entropy. The latter leads to distributions whose entropy is maximal under certain constraints, usually on their moments. The classical von Mises (vM) model with pdf

$$
f_{\Theta}(\theta ; \mu, \kappa)=\frac{1}{2 \pi \mathcal{I}_{0}(\kappa)} \exp \{\kappa \cos (\theta-\mu)\}
$$

where $\mu \in[0,2 \pi)$ is the mean direction, $\kappa>0$ its concentration parameter and $\mathcal{I}_{\nu}$ denotes the modified Bessel function of the first kind and order $\nu$, can be derived using no less than five of these constructions (Mardia and Jupp, 1999, Section 3.5.4). Due to their relevance in the sequel, below we give brief descriptions of wrapping, projection, and perturbation. 
If $X$ is a linear $\mathrm{rv}$ then $\Theta=X(\bmod 2 \pi) \in[0,2 \pi)$ is its wrapped circular counterpart. Alternatively, using a complex representation, $\Theta=\operatorname{Arg}\{\exp (\mathrm{i} X)\} \in[-\pi, \pi)$. If $\phi_{X}$ is the characteristic function (cf) of $X$ then the $\mathrm{cf}$ of $\Theta$ is the set $\left\{\phi_{k}: k=0, \pm 1, \ldots\right\}$ where $\phi_{k}=\mathrm{E}\left(\mathrm{e}^{\mathrm{i} k \Theta}\right)=\phi_{X}(k)$, the $\phi_{k}$ being referred to as the Fourier coefficients or trigonometric moments (Pewsey et al., 2013, Section 4.2.2) of $\Theta$. Thus, $\Theta$ inherits the of of $X$. If $f_{X}$ is the pdf of $X$ then the pdf of $\Theta$ is $f_{\Theta}(\theta)=\sum_{k=-\infty}^{\infty} f_{X}(\theta+2 \pi k)$, the infinite sum generally not reducing to a closed-form expression. An important exception is the pdf of the wrapped Cauchy (WC) distribution,

$$
f_{\Theta}(\theta ; \mu, \rho)=\frac{1}{2 \pi} \frac{1-\rho^{2}}{1+\rho^{2}-2 \rho \cos (\theta-\mu)},
$$

where $\mu=\operatorname{Arg}\left\{\mathrm{E}\left(\mathrm{e}^{\mathrm{i} \Theta}\right)\right\} \in[-\pi, \pi)$ is the mean direction and $\rho=\left|\mathrm{E}\left(\mathrm{e}^{\mathrm{i} \Theta}\right)\right| \in[0,1]$ the mean resultant length. More generally, the trigonometric moments of the WC model are given by

$$
\phi_{k}=\left(\rho \mathrm{e}^{\mathrm{i} \mu}\right)^{k}, \quad k=1,2, \ldots .
$$

Perhaps the best known wrapped model is the wrapped normal distribution, obtained when $X \sim$ $\mathrm{N}\left(\mu, \sigma^{2}\right)$. It can be used to closely approximate the vM distribution, and vice versa (Pewsey and Jones, 2005). Appealing wrapped circular models investigated recently include the wrapped: skewnormal (Pewsey, 2000, 2006), exponential and Laplace (Jammalamadaka and Kozubowski, 2004), $t$ (Pewsey et al., 2007), stable (Pewsey, 2008), and generalized normal-Laplace (Reed and Pewsey, 2009).

Projection involves projecting univariate or bivariate linear random variables onto $\mathbb{S}^{1}$. For example, stereographic projection of the linear random variable $X$ produces the circular $\mathrm{rv} \Theta=2 \tan ^{-1}(X)$ (Abe et al., 2010). Radial projection of a bivariate linear random vector $\boldsymbol{X}=\left(X_{1}, X_{2}\right)$ onto $\mathbb{S}^{1}$ results in the circular rv $\Theta=\operatorname{Arg}\left(X_{1}+\mathrm{i} X_{2}\right)$ or, equivalently, the random point $\boldsymbol{X} /\|\boldsymbol{X}\|$ on $\mathbb{S}^{1}$. Perhaps the best-known distribution of this latter type is the projected normal, also known as offset normal or angular Gaussian, the pdf of which can be symmetric or asymmetric, unimodal or bimodal in shape (Mardia and Jupp, 1999, Section 3.5.6). Projection is a natural construction when modelling measurements relative to the position of an observer.

Perturbation involves multiplying a pdf by a suitable function so as to modulate its shape in some desired way. The cardioid distribution, with pdf

$$
f_{\Theta}(\theta ; \mu, \rho)=\frac{1}{2 \pi}\{1+2 \rho \cos (\theta-\mu)\},
$$

where $|\rho|<1 / 2$, is an example of perturbation of the circular uniform model. Umbach and Jammalamadaka (2009) adapted the perturbation approach of Azzalini (1985) to the circular context, a special case of which is the sine-skewed family of distributions studied by Abe and Pewsey (2011). If $g_{\Theta}$ denotes a base symmetric unimodal circular pdf with mean direction $\mu$ then the pdf of its sine-skewed extension is

$$
f_{\Theta}(\theta ; \mu, \lambda)=g_{\Theta}(\theta-\mu)\{1+\lambda \sin (\theta-\mu)\},
$$

where $\lambda \in[-1,1]$ is a skewing parameter. The symmetric base pdf is unperturbed when $\lambda=0$, otherwise it is skewed in the anticlockwise direction $(\lambda>0)$ or the clockwise direction $(\lambda<0)$. Sine-skewed densities have the same normalising constants as their base symmetric densities, but can model only moderate departures from symmetry and are not necessarily unimodal.

An overarching family of symmetric unimodal circular distributions containing, amongst others, the circular uniform, cardioid, vM, and WC distributions, was proposed by Jones and Pewsey (2005). Its pdf is

$$
f_{\Theta}(\theta ; \mu, \rho, \psi) \propto\{1+\tanh (\kappa \psi) \cos (\theta-\mu)\}^{1 / \psi},
$$


where $\mu \in[0,2 \pi)$ is the mean direction, $\kappa \geq 0$ is a concentration parameter, and $\psi \in \mathbb{R}$ is a shape index.

Recently, Kato and Jones (2015) proposed a highly flexible extension of the WC model obtained by broadening the trigonometric moments in (3) to $\gamma\left(\rho \mathrm{e}^{\mathrm{i} \lambda}\right)^{-1}\left\{\rho \mathrm{e}^{\mathrm{i}(\mu+\lambda)}\right\}^{k}$. The resulting family is unimodal and has pdf

$$
f_{\Theta}(\theta ; \mu, \rho, \gamma, \lambda)=\frac{1}{2 \pi}\left\{1+2 \gamma \frac{\cos (\theta-\mu)-\rho \cos \lambda}{1+\rho^{2}-2 \rho \cos (\theta-\mu-\lambda)}\right\}
$$

where $\mu \in[0,2 \pi), \rho \in[0,1), \gamma \in[0,(1+\rho) / 2]$, and $\lambda \in[-\pi, \pi)$ satisfies $\rho \gamma \cos \lambda \geq\left(\rho^{2}+2 \gamma-1\right) / 2$. Its cdf also has a closed form. Its reparametrisation in terms of standard trigonometric moments (Pewsey, 2004a) has parameters with clear interpretations and is the one generally used to perform inference.

Constructions based on Möbius transformation, Brownian motion, and transformation of argument have also been used recently to generate more flexible families of circular models. A Möbius transformation preserving the unit circle maps a point on the unit circle, $\Theta$, to another, $\Theta^{*}$, via

$$
\mathrm{e}^{\mathrm{i} \Theta^{*}}=\mathrm{e}^{\mathrm{i} \phi} \frac{\mathrm{e}^{\mathrm{i} \Theta}+r \mathrm{e}^{\mathrm{i} \omega}}{r \mathrm{e}^{\mathrm{i}(\Theta-\omega)}+1},
$$

where $\phi, \omega \in[-\pi, \pi)$ and $r \in[0,1)$, or equivalently via

$$
\Theta^{*}=\phi+\omega+2 \tan ^{-1}\left[w_{r} \tan \{(\Theta-\omega) / 2\}\right],
$$

where $w_{r}=(1-r) /(1+r)$. Applying this Möbius transformation to a circular uniform rv results in a WC rv (McCullagh, 1996). Kato and Jones (2010) and Wang and Shimizu (2012) studied families obtained by applying the same transformation to $\mathrm{vM}$ and cardioid random variables, respectively. Jacimovic and Crnkić (2017) related the former family to the dynamics of coupled oscillators. Kato and Jones (2013) varied the Brownian motion specification leading to the WC distribution so as to generate a four-parameter extension of it. The families of Kato and Jones (2010), Wang and Shimizu (2012), and Kato and Jones (2013) have pdfs that can be symmetric or asymmetric and unimodal or bimodal in shape.

Transformation of argument involves replacing the argument of an existing circular pdf, $f_{\Theta}$, by some function of $\theta$. Jones and Pewsey (2012) used this approach to derive inverse Batschelet distributions. The resulting four-parameter distributions are unimodal and highly flexible in shape. Unlike the smooth unimodal models of Kato and Jones (2015), inverse Batschelet distributions can adopt Laplace-like shapes. The most flexible unimodal circular models currently available are those of Jones and Pewsey (2012) and Kato and Jones (2015).

Of the modelling approaches available for multimodal circular data, finite mixtures have proven the most popular. Mixture models with vM components have recently received renewed attention (Mooney et al. (2003); Fu et al. (2008); see, also, Section 3.4). The pdf of an $m$ component vM mixture is

$$
f_{\Theta}(\theta ; \boldsymbol{p}, \boldsymbol{\mu}, \boldsymbol{\kappa})=\sum_{j=1}^{m} p_{j} f_{\Theta}\left(\theta ; \mu_{j}, \kappa_{j}\right),
$$

where $\boldsymbol{p}=\left(p_{1}, \ldots, p_{m}\right)^{\prime}$ is a vector of mixing probabilities satisfying $\sum_{j=1}^{m} p_{j}=1, \boldsymbol{\mu}=\left(\mu_{1}, \ldots, \mu_{m}\right)^{\prime}$, $\boldsymbol{\kappa}=\left(\kappa_{1}, \ldots, \kappa_{m}\right)^{\prime}$, and $f_{\Theta}\left(\theta ; \mu_{j}, \kappa_{j}\right)$ is as in $(2)$. When the interpretation of the parameters of the component densities is straightforward, so is the interpretation of the parameters of a mixture. 
More generally, Holzmann et al. (2004) established conditions for the identifiability of mixtures of location-scale extensions of wrapped circular models including the wrapped symmetric $\alpha$-stable, wrapped normal, and WC distributions. Mixtures with circular triangular (McVinish and Mengersen, 2008), skew-rotationally symmetric (Miyata et al., 2019), and power Batschelet (Mulder et al., 2020b) components have also been considered.

Finally, we consider three alternative approaches to modelling multimodal circular data. Generalized von Mises models (Gatto, 2008, 2009), with the density of the generalized vM distribution of order $m$ being

$$
f_{\Theta}(\theta ; \boldsymbol{\mu}, \boldsymbol{\kappa})=\exp \left\{\kappa_{0}+\sum_{j=1}^{m} \kappa_{j} \cos \left(j\left(\theta-\mu_{j}\right)\right)\right\},
$$

where $\boldsymbol{\mu}=\left(\mu_{1}, \ldots, \mu_{m}\right)^{\prime}, \boldsymbol{\kappa}=\left(\kappa_{1}, \ldots, \kappa_{m}\right)^{\prime}, \mu_{j} \in[0,2 \pi / j)$, and $\kappa_{j} \geq 0$, have a long history dating back to Maksimov (1967). The normalising constant, $\mathrm{e}^{\kappa_{0}}$, must generally be computed numerically. Fernández-Durán (2004) revisited work by Fejér (1916) when defining a family of circular distributions based on non-negative trigonometric (i.e. truncated Fourier) sums. Whilst they do not require the calculation of normalising constants, fitted densities of this type tend to have many parameters and display minor harmonic modes that need not be supported by the data. Recently, Taniguchi et al. (2020) proposed flexible models for circular data obtained by normalising the spectra of stochastic processes, the residue theorem being used to calculate their normalising constants. The interpretation of the parameters of all three of these types of model is, however, generally difficult.

\subsection{Models for toroidal data}

Let $\left(\Theta_{1}, \Theta_{2}\right)$ denote the angular coordinates of a random vector distributed on the torus $\mathbb{T}^{2}=\mathbb{S}^{1} \times \mathbb{S}^{1}$. Some of the approaches used to generate models for toroidal data are extensions of those introduced in Section 3.1. These include maximum entropy characterisation, projection, and wrapping (Johnson and Wehrly, 1977; Baba, 1981; Mardia et al., 2008). Models for univariate and bivariate axial data were proposed by Arnold and SenGupta (2006).

The bivariate von Mises model of Mardia (1975) is a maximum entropy (equivalently, an exponential family) distribution with pdf

$$
\begin{aligned}
f_{\Theta_{1}, \Theta_{2}}\left(\theta_{1}, \theta_{2}\right) \propto & \exp \left\{\kappa_{1} \cos \left(\theta_{1}-\mu_{1}\right)+\kappa_{2} \cos \left(\theta_{2}-\mu_{2}\right)\right. \\
& \left.+\left(\cos \left(\theta_{1}-\mu_{1}\right), \sin \left(\theta_{1}-\mu_{1}\right)\right) \boldsymbol{A}\left(\cos \left(\theta_{2}-\mu_{2}\right), \sin \left(\theta_{2}-\mu_{2}\right)\right)^{\prime}\right\}
\end{aligned}
$$

where $\mu_{1}, \mu_{2} \in[-\pi, \pi), \kappa_{1}, \kappa_{2} \geq 0$, and $\boldsymbol{A}$ is a $2 \times 2$ matrix. The most compact form for its normalising constant involves a doubly infinite sum (Mardia, 2010). The model has eight parameters, three more than the minimum of five required to control the locations and concentrations of the two marginal variables and the dependence between them. Moreover, their interpretation is difficult (Mardia et al., 2007). In the search for five-parameter analogous of the bivariate normal distribution, Singh et al. (2002), Mardia et al. (2007), and Kent et al. (2008) proposed the sine, cosine, and hybrid submodels of (8), respectively. The properties of these three submodels were compared in Kent et al. (2008) and Mardia and Frellsen (2012). Their normalising constants are available as infinite sums. Their conditional distributions are vM, but their marginal pdfs are generally not and, for some parameter values, can be bimodal.

Extensions of (8) and its cosine submodel, for use with data on $\mathbb{T}^{d}, d \geq 2$, were proposed by Mardia et al. (2008) and Mardia and Patrangenaru (2005), respectively. No simple closed analytic form is generally available for the normalising constant of the sine multivariate von Mises model of Mardia 
et al. (2008), but its conditional distributions are vM and thus its parameters can be estimated by maximising the pseudo-likelihood. Conditions on its parameters to ensure unimodality were established in Mardia and Voss (2014) and pseudo-likelihood regularised approaches were given in Rodriguez-Lujan et al. (2015, 2017). A multivariate extension of the second-order generalized vM $\left(\mathrm{GvM}_{2}\right)$ distribution (with $m=2$ in $(7)$ ), obtained by conditioning a multivariate Gaussian distribution on $\mathbb{R}^{2 d}$ to $\mathbb{T}^{d}$, was proposed by Navarro et al. (2017). Its one-dimensional conditional distributions are $\mathrm{GvM}_{2}$, and the sine multivariate vM model is a special case of it. Hassanzadeh and Kalaylioglu (2018) recently proposed a model for data on $\mathbb{T}^{2}$ obtained using a conditional specification construction involving $\mathrm{GvM}_{2}$ pdfs.

The range of available models can be expanded beyond toroidal analogues of the bivariate normal distribution using the projection approach of Saw (1983) to construct models with more flexible specified marginal distributions. A simpler marginal specification approach can be traced back to Wehrly and Johnson (1980). They proposed toroidal pdfs of the form

$$
f_{\Theta_{1}, \Theta_{2}}\left(\theta_{1}, \theta_{2}\right)=2 \pi f_{\Theta_{1}}\left(\theta_{1}\right) f_{\Theta_{2}}\left(\theta_{2}\right) f_{\Omega}\left(2 \pi\left[F_{\Theta_{2}}\left(\theta_{2}\right)-q F_{\Theta_{1}}\left(\theta_{1}\right)\right]\right)
$$

where $f_{\Theta_{j}}$ and $F_{\Theta_{j}}$ are the marginal pdf and cdf of $\Theta_{j}, j=1,2, f_{\Omega}$ is a circular binding pdf, and $q= \pm 1$ determines whether the dependence is positive or negative. Various models obtained using (9) with different choices for $f_{\Theta_{1}}, f_{\Theta_{2}}$, and $f_{\Omega}$ are referred to in Jones et al. (2015).

Kato and Pewsey (2015) considered a case of (9) having a closed-form pdf that is unimodal and pointwise symmetric, and marginal and conditional distributions that are all WC. This bivariate WC model can also be obtained by applying a Möbius transformation to a tractable toroidal model with circular uniform marginal distributions derived by Kato (2009) using a Brownian motion construction.

Shieh and Johnson (2005) were the first to note the relationship between (9) and copulas (Sklar, 1959), toroidal pdfs being generated through

$$
f_{\Theta_{1}, \Theta_{2}}\left(\theta_{1}, \theta_{2}\right)=f_{\Theta_{1}}\left(\theta_{1}\right) f_{\Theta_{2}}\left(\theta_{2}\right) c\left(F_{\Theta_{1}}\left(\theta_{1}\right), F_{\Theta_{2}}\left(\theta_{2}\right)\right),
$$

where $c$ is a copula pdf. García-Portugués et al. (2013a) imposed periodic restrictions on $c$ to construct alternatives to (9). Jones et al. (2015) revisited (9) and considered, instead of (10),

$$
f_{\Theta_{1}, \Theta_{2}}\left(\theta_{1}, \theta_{2}\right)=4 \pi^{2} f_{\Theta_{1}}\left(\theta_{1}\right) f_{\Theta_{2}}\left(\theta_{2}\right) c_{\circ}\left(2 \pi F_{\Theta_{1}}\left(\theta_{1}\right), 2 \pi F_{\Theta_{2}}\left(\theta_{2}\right)\right),
$$

where now $c_{\circ}$ is what they coined a circula density, with arguments that are circular uniform. For any circula density, $c_{\circ}\left(\theta_{1}, \theta_{2}\right)=c_{\circ}\left(\theta_{1} \pm 2 \pi k, \theta_{2} \pm 2 \pi l\right), k, l \in \mathbb{Z}^{+}$. Jones et al. (2015) showed that (9) corresponds to $c_{\circ}\left(\phi_{1}, \phi_{2}\right)=\frac{1}{2 \pi} f_{\Omega}\left(\phi_{2}-q \phi_{1}\right)$, the pdf of $\left(\Phi_{1}, \Phi_{2}\right)$, where $\Phi_{1}$ and $\Phi_{2}=\Phi_{1}-q \Omega(\bmod 2 \pi)$ are circular uniform random variables and $\Omega$ follows the circular pdf $f_{\Omega}$ independently of $\Phi_{1}$. This circula density is a particular case of the (infinite) Fourier series approach to obtaining circula densities proposed recently by Kato et al. (2018). They considered six cases of their general construction, all having simple closed-form expressions for their densities. Jupp (2015) extended the idea of circulas to compact Riemannian manifolds.

Recently, Ameijeiras-Alonso and Ley (2019) used a sine-skewing approach (see Section 3.1) to generate models for asymmetric data on $\mathbb{T}^{d}, d \geq 2$. Alternative approaches to modelling such data make use of pdfs obtained from truncated Fourier series (Pertsemlidis et al., 2005; Fernández-Durán and Gregorio-Domínguez, 2014b) or normalised spectra of stochastic processes (Taniguchi et al., 2020). These models have properties analogous to those mentioned in Section 3.1 for their circular counterparts. 


\subsection{Models for cylindrical data}

Let $(\Theta, X)$ denote the coordinates of a random vector distributed on the cylinder $\mathbb{S}^{1} \times \mathbb{R}$. Approaches used to generate models for cylindrical data have included wrapping (Johnson and Wehrly, 1977), conditioning, marginal specification, and maximum entropy characterisation.

Mardia and Sutton (1978) conditioned a trivariate normal distribution to obtain a six-parameter cylindrical model for which the marginal distribution of $\Theta$ is $\mathrm{vM}$ and the conditional distribution of $X \mid \Theta=\theta$ is normal. More recently, Kato and Shimizu (2008) proposed an eight-parameter extension of it having generalized vM distributions for $\Theta$ and $\Theta \mid X=x$.

An analogous marginal specification approach to that used to derive pdf (9) can be employed to obtain a cylindrical model with pdf

$$
f_{\Theta, X}(\theta, x)=2 \pi f_{\Theta}(\theta) f_{X}(x) f_{\Omega}\left(2 \pi\left[F_{\Theta}(\theta)-q F_{X}(x)\right]\right),
$$

where $f_{\Theta}$ and $f_{X}$ are the marginal pdfs of $\Theta$ and $X$, respectively, and $F_{\Theta}$ and $F_{X}$ their cdfs. Johnson and Wehrly (1978) considered cases of (11) with $X$ normally distributed, and circular uniform or vM distributions for $\Theta$. Recently, other cases have been applied to model cylindrical data from disciplines such as wind energy analysis (Carta et al., 2008; Zhang et al., 2018), ocean engineering (Soukissian, 2014), and image analysis (Roy et al., 2017).

Johnson and Wehrly (1978) also proposed three maximum entropy cylindrical models, with conditional distributions that are $\mathrm{vM}$ and normal or exponential. The dependence structures of all three models are, however, severely constrained. Their model having vM and exponential marginal distributions when $\Theta$ and $X$ are independent was recently extended by Abe and Ley (2017), Imoto et al. (2019), and Abe and Shimatani (2018) so as to admit skew and more flexible models for $\Theta$ and $X$, respectively.

Recently, Mastrantonio (2018) proposed the joint projected normal and skew-normal distribution, the first model for multivariate cylindrical data. It is highly flexible and closed under marginalisation.

\subsection{Models for spherical data}

Suppose $\boldsymbol{X}$ is a unit random vector on $\mathbb{S}^{d}$. Perhaps the best-known model for spherical data is the von Mises-Fisher (vMF) distribution, with pdf

$$
f_{\boldsymbol{X}}(\boldsymbol{x} ; \boldsymbol{\mu}, \kappa)=\frac{\kappa^{(d-1) / 2}}{(2 \pi)^{(d+1) / 2} \mathcal{I}_{(d-1) / 2}(\kappa)} \exp \left\{\kappa \boldsymbol{x}^{\prime} \boldsymbol{\mu}\right\}
$$

where $\boldsymbol{\mu} \in \mathbb{S}^{d}$ is the mean direction vector and $\kappa \geq 0$ is a concentration parameter. Other classical models are the Kent, Fisher-Watson, Bingham-Mardia, Bingham, and Watson distributions (Mardia and Jupp, 1999, Chapter 9), the last two being models for axial data. They are all submodels of the Fisher-Bingham exponential family of distributions with pdf

$$
f_{\boldsymbol{X}}(\boldsymbol{x} ; \boldsymbol{\mu}, \kappa, \boldsymbol{A}) \propto \exp \left\{\kappa \boldsymbol{x}^{\prime} \boldsymbol{\mu}+\boldsymbol{x}^{\prime} \boldsymbol{A} \boldsymbol{x}\right\},
$$

where $\boldsymbol{A}$ is a symmetric $(d+1) \times(d+1)$ matrix, and $\boldsymbol{\mu}$ and $\kappa$ play the same roles as in (12). Evaluation of the distribution's normalising constant is challenging but Kume and Sei (2018) showed how it can be achieved using the holonomic gradient method. Kent et al. (2018) developed an efficient acceptance-rejection method of simulating variates from Fisher-Bingham distributions on spheres and related manifolds. Kent et al. (2016) introduced a five-parameter special case of the Fisher-Bingham model for use with data patterns that are unimodal and concentrated near a great 
circle. More recently, Kim et al. (2019) proposed two kinds of small-sphere distributions, one of which is a member of the Fisher-Bingham family. Previously, Oualkacha and Rivest (2009) had developed an alternative to the Bingham distribution for modelling symmetric axial data, with a simple closed-form normalising constant.

Rotationally symmetric (RS) spherical pdfs depend on $\boldsymbol{x}$ only through $\boldsymbol{x}^{\prime} \boldsymbol{\mu}$ and, as a consequence, have contours that are circular when $\boldsymbol{x} \in \mathbb{S}^{2}$. Historically, the vMF has been the most important such model. In recent years, numerous other RS families have been proposed in the literature: Section 2.3.2 of Ley and Verdebout (2017a) summarises many of them. The spherical logistic distribution of Moghimbeygi and Golalizadeh (2020) provides a multimodal and RS extension of the vMF, with a closed-form normalising constant when $d=2$. Another recent addition is the highly tractable spherical Cauchy distribution of Kato and McCullagh (2020), which extends the WC to $\mathbb{S}^{d}$ and has a very simple normalising constant.

The pdf of the Kent distribution, i.e. (13) constrained to have $\boldsymbol{A} \boldsymbol{\mu}=\mathbf{0}$, has elliptical contours and hence can be used to model certain departures from isotropy. Recently, Paine et al. (2018) proposed the elliptically symmetric angular Gaussian (ESAG) as an alternative. As simulation from it and the computation of its pdf are far quicker than for the Kent model, this model is a particularly appealing alternative when the use of computer intensive methods is being contemplated. Other Kent-like alternatives with the advantages of the ESAG model are the scaled vMF family of Scealy and Wood (2019), which has an additional parameter controlling tail-weight, and the tangent models of García-Portugués et al. (2020b).

The sine-skewed circular distributions of Section 3.1 are special cases of the skew-rotationally symmetric (SRS) distributions proposed as models for asymmetric spherical data by Ley and Verdebout (2017b). In turn, SRS models are spherical analogues of the skew-symmetric linear models of Wang et al. (2004). The perturbation of spherical distributions was studied in greater generality by Jupp et al. (2016).

Asymmetric or bimodal spherical data can be modelled using the general projected normal family of distributions referred to in Mardia and Jupp (1999, Section 9.3.3) and advocated more recently from a Bayesian perspective in Hernandez-Stumpfhauser et al. (2017). Núñez-Antonio and Geneyro (2020) proposed a projected gamma distribution to model data on the positive orthant of $\mathbb{S}^{d}$.

In Section 9 we summarise recent developments in the use of mixture distributions with spherical component pdfs as a means of modelling multimodal spherical data. The flexible directional logspline pdfs of Ferreira et al. (2008), based on thin-plate splines on $\mathbb{S}^{d}$ (Taijeron et al., 1994), provide an alternative means of modelling multimodality and skewness. They are given by

$$
f_{\boldsymbol{X}}(\boldsymbol{x} ; \boldsymbol{c}, \mathcal{K}, m)=\exp \left\{c_{0}+\sum_{j=1}^{m} c_{j} R_{d}\left(\boldsymbol{x} ; \boldsymbol{k}_{j}\right)\right\}
$$

where $m \geq 1,\left(c_{0}, c_{1}, \ldots, c_{m}\right)^{\prime} \in \mathbb{R}^{m+1}, \mathcal{K}=\left\{\boldsymbol{k}_{1}, \ldots, \boldsymbol{k}_{m}\right\}$ is a set of knot vectors in $\mathbb{S}^{d}$, and $R_{d}\left(\cdot ; \boldsymbol{k}_{j}\right)$ are real-valued spline basis functions on $\mathbb{S}^{d}$ that, when evaluated at $\boldsymbol{x} \in \mathbb{S}^{d}$, are functions of $\boldsymbol{x}^{\prime} \boldsymbol{k}_{j}$, for $j=1, \ldots, m$. Ferreira et al. (2008) proposed a Bayesian inferential approach for (14). FernándezDurán and Gregorio-Domínguez (2014a) constructed pdfs on $\mathbb{S}^{2}$ through non-negative trigonometric sum expansions in terms of spherical angles. 


\section{General approaches to inference}

Historically, inference for the models in Section 3 has generally been frequentist: sometimes using the method of (trigonometric) moments but more generally being likelihood-based. The maximum likelihood (ML) estimators of full exponential family models are moment estimators (van der Vaart, 2000, Chapter 4) and, as a consequence, closed-form expressions exist for the ML estimators of, for example, the vM distribution and the cylindrical model of Mardia and Sutton (1978). Exact ML inference for the highly challenging Fisher-Bingham model and its submodels identified in Section 3.4 was developed recently in Kume and Sei (2018). More generally, maximisation of the log-likelihood has to be performed numerically. When available, method of moments estimates can be used as starting values for that process. For some models, statistical inference based on the full log-likelihood is intractable and pseudo-likelihood methods have been employed (Kent et al., 2008). Score matching estimators, inspired by the Hyvärinen (2005) scoring rule, circumvent the need to calculate normalising constants for directional distributions (Mardia et al., 2016; Mardia, 2018; Takasu et al., 2018).

Large-sample results for ML-based inference generally assume standard regularity conditions to apply and the asymptotic normality of ML estimators (van der Vaart, 2000, Chapter 5). Pewsey (2004a) employed the delta method to obtain the asymptotic distribution of the fundamental measures of central location, concentration, skewness, and kurtosis used in the analysis of circular data. For some models, large-sample ML-based inference for parameter values on the boundary of the parameter space will be of interest, and the results of Self and Liang (1987) can be employed (see, e.g., Shieh and Johnson, 2005). For small-sized samples, bootstrap confidence interval constructions have become increasingly popular (Pewsey et al., 2013, Chapter 5). Computer intensive resampling methods in hypothesis testing are mentioned in Section 5.

Recently, Le Cam's local asymptotic normality approach to inference has been adapted to problems in directional statistics: see Section 5, and Section 5 of Ley and Verdebout (2017a). The first such adaptation appeared in Ley et al. (2013), where optimal rank-based estimators of the location parameter of rotationally symmetric spherical distributions were proposed. More recently, Paindaveine and Verdebout (2020) considered inference under high concentration for the spherical location of a semi-parametric class of rotationally symmetric distributions.

Bayesian inferential techniques have become increasingly popular in recent years, often being implemented using Markov chain Monte Carlo (MCMC) methods. A general approach to MCMC simulation on embedded Riemannian manifolds was introduced by Byrne and Girolami (2013), and illustrated for the Fisher-Bingham distribution. Bayesian approaches to inference have been developed for the: vM (Damien and Walker, 1999) and mixtures thereof (Mulder et al., 2020a), WC (Ghosh et al., 2019), vMF (Núñez-Antonio and Gutiérrez-Peña, 2005b; Hornik and Grün, 2013), bivariate vM (Mardia, 2010), and projected normal (Núñez-Antonio and Gutiérrez-Peña, 2005a) distributions. Bhattacharya and SenGupta (2009b) considered Bayesian inference for circular distributions with unknown normalising constants. Fallaize and Kypraios (2016) gave a Monte Carlo exact Bayesian method of inference for the Bingham distribution. Bayesian approaches based on projected and wrapped models have become popular for a range of applications: see Sections 3.4 and 11. Scoring rules provide an alternative to the traditional Bayesian formulation, and have been applied for the vMF distribution (Giummolè et al., 2019).

Robust estimators have been proposed for the parameters of the vM and wrapped normal distributions (Agostinelli, 2007), the vMF distribution (Kato and Eguchi, 2016), and a range of other circular distributions in a series of papers referred to by Laha et al. (2019). 
Asymptotic results for extrinsic and intrinsic means on manifolds, including $\mathbb{S}^{d}$, were obtained in Bhattacharya and Patrangenaru (2003, 2005); see Bhattacharya and Patrangenaru (2014) for a review on the topic. Hotz (2013) gave a detailed comparison between extrinsic and intrinsic means on $\mathbb{S}^{1}$. Nonparametric inference on intrinsic means on circles and spheres, however, can be fundamentally different from its Euclidean analogues due to the effect of smeariness (asymptotic rates are slower than $n^{-1 / 2}$ ) present on $\mathbb{S}^{1}$ (Hotz and Huckemann, 2015) and $\mathbb{S}^{d}$ (Eltzner and Huckemann, 2019). The related effect of finite sample smeariness has been studied on the circle by Hundrieser et al. (2020) and may affect all of the circular distributions mentioned previously. As a consequence, quantile-based tests may be inappropriate while suitable bootstrap tests remain valid.

The inferential impact of the reference systems used for circular distributions was explored recently in Mastrantonio et al. (2019).

\section{$5 \quad$ Hypothesis testing}

Here we consider hypothesis tests for uniformity, symmetry, location, concentration, goodness-of-fit, and other testing scenarios. Calibration of the tests has generally been based on asymptotic theory and, for small to moderate sized samples, the use of resampling methods.

\subsection{Uniformity}

Uniformity (or isotropy), corresponding to there being no preferred direction, is the most important dividing hypothesis in directional statistics. García-Portugués and Verdebout (2018) provide an extensive review of tests for it.

Sobolev tests (Beran, 1968, 1969; Giné, 1975) form, by far, the most extensive class of tests for uniformity on $\mathbb{S}^{d}$. Given a sample $\boldsymbol{X}_{1}, \ldots, \boldsymbol{X}_{n}$ on $\mathbb{S}^{d}$, Sobolev statistics take the form

$$
S_{n}\left(\left\{v_{k}^{2}\right\}\right)=\frac{1}{n} \sum_{i, j=1}^{n} \sum_{k=1}^{\infty} v_{k}^{2} h_{k}\left(\boldsymbol{X}_{i}, \boldsymbol{X}_{j}\right),
$$

where

$$
h_{k}(\boldsymbol{u}, \boldsymbol{v})=\left\{\begin{array}{l}
2 \cos \left(k \cos ^{-1}\left(\boldsymbol{u}^{\prime} \boldsymbol{v}\right)\right), \quad d=1, \\
\left(1+\frac{2 k}{d}\right) C_{k}^{(d-1) / 2}\left(\boldsymbol{u}^{\prime} \boldsymbol{v}\right), \quad d>1,
\end{array}\right.
$$

$C_{k}^{(d-1) / 2}$ is the $k$-th Gegenbauer polynomial of index $(d-1) / 2$, and the $v_{k}^{2}$ should decay fast enough to ensure convergence in (15). Different choices for $\left\{v_{k}^{2}\right\}$ give different local optimality properties, consistencies, and powers against specific kinds of alternatives. For example, the choices $v_{k}=\delta_{k j}$, $j=1,2$, give, respectively, the test statistics of Rayleigh (1919) and Bingham (1974). Both were modified by Jupp (2001) to improve their convergence under the null hypothesis. The alternatives for which the Rayleigh and Bingham tests are inconsistent were identified by Ehler and Galanis (2011) as the minimisers of certain potentials over $\mathbb{S}^{d}$. The Rayleigh test plays a key role in the CUSUM-based test for circular uniformity developed by Lombard and Maxwell (2012). Pycke (2007, 2010 ) proposed uniformity tests on $\mathbb{S}^{2}$ and $\mathbb{S}^{1}$ based on the geometric mean of pairwise chordal distances, whilst Bakshaev (2010) gave an analogous approach based on the arithmetic mean. The Bayesian optimality of Sobolev tests on $\mathbb{S}^{1}$ was studied by Sun and Lockhart (2019).

"Data-driven" Sobolev tests are obtained by using an information criterion to truncate the infinite series in (15). This approach was used to obtain tests of uniformity on $\mathbb{S}^{1}$ by Bogdan et al. (2002), and on compact Riemannian manifolds by Jupp (2008, 2009). Such truncation simplifies the computation of (15) and its asymptotic distribution, the latter effectively being chi-squared. A variation on 
this approach was pursued recently by Jammalamadaka et al. (2020), who proposed increased levels of truncation of $(15)$ on $\mathbb{S}^{1}$ and $\mathbb{S}^{2}$, so as to obtain a normal limit rather than the usual weighted sum of chi-squared random variables appearing in the asymptotic null distribution of (15).

$\mathrm{Su}$ and $\mathrm{Wu}$ (2011) considered spherical harmonics and exponential models as alternatives to uniformity, and derived score tests strongly related to Sobolev tests to test for uniformity against them. Also related to Sobolev tests, García-Portugués et al. (2020a) proposed a class of tests based on the projected empirical cdf that yields extensions for data on $\mathbb{S}^{d}$ of the Watson (1961) and Rothman (1972) tests for circular uniformity, and a novel Anderson-Darling-like test for uniformity on $\mathbb{S}^{d}$.

Recent non-Sobolev tests for circular uniformity include the four-point Cramér-von Mises test of Feltz and Goldin (2001), the likelihood-ratio test against a mixture with symmetric wrapped stable and circular uniform components of SenGupta and Pal (2001), the spacings-based Gini mean difference test of Tung and Jammalamadaka (2013), and the Bayesian tests of Mulder and Klugkist (2021) against the vM distribution and the kernel density estimator (18). Tests for uniformity on $\mathbb{S}^{d}$ include that of Faÿ et al. (2013), based on needlets (see Section 7.1.2), and those of Lacour and Pham Ngoc (2014) and Kim et al. (2016) for "noisy" data on $\mathbb{S}^{2}$, i.e. where the density of the observations is a convolution of an error pdf with a true underlying pdf. Cuesta-Albertos et al. (2009) proposed a projection-based test, and Ebner et al. (2018) one based on a sum of weighted nearest-neighbour distances. Cutting et al. (2020) investigated tests for uniformity on $\mathbb{S}^{d}$ against axial alternatives.

Tests for uniformity when $d \rightarrow \infty$ as $n \rightarrow \infty$ are scarcer. Cai and Jiang (2012) and Cai et al. (2013) proposed tests based on $\max _{i<j}\left|\boldsymbol{X}_{i}^{\prime} \boldsymbol{X}_{j}\right|$. Paindaveine and Verdebout (2016) and Cutting et al. (2017a) studied a standardised Rayleigh statistic under uniformity and vMF alternatives, respectively.

Simulation studies comparing the performance of various tests for uniformity on $\mathbb{S}^{1}$ have been carried out by Landler et al. (2018), on $\mathbb{S}^{d}, d \geq 1$, by García-Portugués et al. (2020a), and on $\mathbb{S}^{d}, d \geq 2$, by Figueiredo and Gomes (2003) and Figueiredo (2007). Humphreys and Ruxton (2017) and Landler et al. (2019) performed simulation experiments to compare the performance of tests for circular uniformity when the data are grouped.

\subsection{Symmetry}

There are at least four forms of symmetry that might be of interest in the analysis of circular data: reflective symmetry about an unknown direction, reflective symmetry about a known median axis, reflective symmetry about a specified median axis, and $\ell$-fold symmetry. Pewsey (2002, 2004b) described these various forms of symmetry and proposed simple, trigonometric moment-based, omnibus tests for the first two scenarios. More recently, Ameijeiras-Alonso et al. (2020), Meintanis and Verdebout (2019), and Ley and Verdebout (2014b) proposed tests for the same two setups that are optimal against the $k$-sine-skewed models of Umbach and Jammalamadaka (2009).

As we saw in Section 3.4, many of the models for spherical data are rotationally symmetric. Recently, García-Portugués et al. (2020b) developed semi-parametric tests for rotational symmetry when the axis of symmetry is known or unknown. Previously, Ley and Verdebout (2017b) had developed two tests for rotational symmetry about a known centre within the class of skew-rotationally symmetric distributions.

For data on $\mathbb{S}^{2}$, Jammalamadaka and Terdik (2019) proposed tests for various types of symmetry, as well as uniformity, based on spherical harmonics. 


\subsection{Location and concentration}

Various tests for the parameters of $\mathrm{vM}$ and $\mathrm{vMF}$ models have been developed in recent years. Larsen et al. (2002) proposed improved likelihood-ratio tests for the: (i) mean direction of a vM distribution; (ii) equality of mean directions of two vM distributions; (iii) concentration of a vMF distribution; (iv) equality of concentrations of two vMF distributions. Watamori and Jupp (2005) introduced improved likelihood-ratio and score tests for homogeneity of concentration in vMF distributions. Their score tests were derived and studied from an alternative perspective in the review of Ley and Verdebout (2014a). Laha and Mahesh (2015) investigated the robustness of tests for the locations of the vM and vMF models. Gatto (2017) proposed a simultaneous test for the mean direction and concentration of a vMF distribution. For data from vMF distributions with a common unknown concentration, Rumcheva and Presnell (2017) proposed an improved version of the multi-sample likelihood-ratio test for the equality of mean directions.

Widening the scope to rotationally symmetric spherical distributions, Tsai (2009) introduced asymptotically efficient rank tests for the equality of the modal direction vectors of two unimodal rotationally symmetric spherical distributions. Ley et al. (2015) investigated the high dimensional robustness of Watson's test for the mean direction. Paindaveine and Verdebout (2015) proposed optimal rankbased tests for the mean direction, and Ley et al. (2017) used the invariance principle to construct rank-based semi-parametric tests for the homogeneity of mean directions. Paindaveine and Verdebout (2017) investigated the problem of testing for a specified mean direction when the underlying distribution tends to uniformity. Cutting et al. (2017b) proposed tests for concentration in low and high dimensions, and Verdebout $(2015,2017)$ tests for homogeneity of concentration.

A simultaneous saddlepoint test for the mean direction and dispersion of the wrapped symmetric stable model was proposed in Gatto (2000), and a nonparametric extension of it, for an assumed underlying unimodal circular distribution, in Gatto (2006). Amaral et al. (2007) proposed nonparametric bootstrap and permutation tests for the equality of the mean directions of directional distributions.

For axial data, Figueiredo (2017) proposed and explored the performance of bootstrap and permutation counterparts of a high-concentration test for the homogeneity of principal axes of Watson distributions.

\subsection{Goodness-of-fit}

Given an independent and identically distributed circular sample, $\Theta_{1}, \ldots, \Theta_{n}$, with underlying cdf $F$, the goodness-of-fit testing problem of $H_{0}: F=F_{0}$ versus $H_{1}: F \neq F_{0}$, where $F_{0}$ is a fully specified cdf, can be addressed using tests for circular uniformity. Appealing to the probability integral transform, testing $H_{0}$ is equivalent to testing the sample $2 \pi F_{0}\left(\Theta_{1}\right), \ldots, 2 \pi F_{0}\left(\Theta_{n}\right)$ on $[0,2 \pi)$ for circular uniformity. When the parameters of a model are estimated, the sampling distributions of the test statistics are affected. However, those sampling distributions can be approximated using the parametric bootstrap (Pewsey et al., 2013, Chapter 6).

For data on other supports, the situation is more complicated as there is no canonical transformation to uniformity. Examples of bootstrap goodness-of-fit tests for models fitted to toroidal data appear in Jones et al. (2015), Pewsey and Kato (2016), and Kato et al. (2018): the approach used in the latter essentially being based on the multivariate probability integral transform. Almost-canonical transformations for other Riemannian manifolds have been proposed recently in Jupp and Kume (2020). 
Using spherical harmonic expansions, Boulerice and Ducharme (1997) developed goodness-of-fit tests for the vMF and Watson distributions. Jupp (2005) considered weighted Sobolev goodnessof-fit tests for distributions on compact Riemannian manifolds. Deschepper et al. (2008) proposed a lack-of-fit test for linear-circular regression models based on the arcs generated by the circular observations. Wouters et al. (2009) proposed data-driven goodness-of-fit tests for the vM model based on orthonormal polynomials.

Smoothing-based approaches to testing the goodness-of-fit of parametric models to directional data have also been developed. Boente et al. (2014) and García-Portugués et al. (2015) used kernel density estimators (see Section 7.1) to test the goodness-of-fit of spherical, and spherical-linear/spherical models, respectively. In the regression context, García-Portugués et al. (2016) proposed a goodnessof-fit test for linear-spherical models based on an extension of (19).

\subsection{Other testing scenarios}

For bivariate circular data, the asymptotic sampling properties of likelihood-ratio tests of independence were considered by Shieh and Johnson (2005), and their permutation analogues by Kato and Pewsey (2015) and Kato et al. (2018). Nonparametric, kernel-based, tests for the independence of spherical-linear/spherical variables were proposed in García-Portugués et al. (2014, 2015).

Several tests for change-point detection in circular series have been introduced. Nonparametric tests include the permutation test of Byrne et al. (2009) and the CUSUM test of Lombard et al. (2017). In vM-distributed series, likelihood (Ghosh et al., 1999; Hawkins and Lombard, 2015) and CUSUM (Hawkins and Lombard, 2017) procedures have been advocated. Bayesian approaches have also been considered (Ghosh et al., 1999; SenGupta and Laha, 2008).

Other tests for circular data include the kernel density estimate-based tests of Fisher and Marron (2001) and Ameijeiras-Alonso et al. (2019a) for assessing the number of modes, and the test of Ducharme et al. (2012) for detecting vortices in two-dimensional vector fields. Recently, bootstrapbased tests using smoothing have been introduced in Zhang et al. (2019), for comparing two samples, and in Alonso-Pena et al. (2019), for testing in circular regression.

\section{Correlation and regression}

\subsection{Correlation}

Mardia and Jupp (1999, Section 11.2) provide details of some of the correlation coefficients for toroidal, cylindrical, and spherical data that have been proposed in the literature. Others are considered in Jammalamadaka and SenGupta (2001, Chapter 8). Recently, Zhan et al. (2019) reviewed the correlation coefficients available for toroidal data and proposed two new ones. In the context of Bayesian network modelling, Leguey et al. (2019b) and Leguey et al. (2019a) introduced mutual information measures of the dependence between circular and linear variables, and between two circular variables, respectively.

\subsection{Regression}

Here we consider parametric regression models. Throughout, we use the generic notation $\mathcal{Y}-\mathcal{X}$ to denote that $\mathcal{Y}$ is the response variable and $\mathcal{X}$ is the explanatory variable. Recent developments in nonparametric regression are described in Section 7.2. 


\subsubsection{Circular-circular regression}

Circular-circular regression is used to model the relationship between a circular response variable, $\Psi$, and a circular explanatory variable, $\Theta$. Polsen and Taylor (2015) reviewed parametric circularcircular regression models and considered inference and diagnostic analysis for them. They focused on the general inverse tangent link-based regression model

$$
\Psi=\operatorname{Arg}\left\{g_{1}(\Theta ; \boldsymbol{\eta})+\mathrm{i} g_{2}(\Theta ; \boldsymbol{\eta})\right\}+\varepsilon(\bmod 2 \pi),
$$

where the first term represents the conditional mean direction of $\Psi$ given $\Theta, g_{1}$ and $g_{2}$ are nonuniquely identifiable functions, $\boldsymbol{\eta}$ is a vector of parameters, and $\varepsilon$ is a circular error variable. The decentred model of Rivest (1997) and the Möbius transformation-based models of Downs and Mardia (2002), Kato et al. (2008), and Kato and Jones (2010) are all special cases of (16). The first two have vM errors, whilst those of the models of Kato et al. (2008) and Kato and Jones (2010) are WC and a four-parameter highly flexible unimodal extension of the WC different from (4), respectively. Polsen and Taylor (2015) also related the bivariate regression model of Sarma and Jammalamadaka (1993) (see also Jammalamadaka and SenGupta, 2001, Section 8.6), having finite-order trigonometric polynomials for $g_{1}$ and $g_{2}$, to the model in (16).

McMillan et al. (2013) proposed a hierarchical Bayesian approach for repeated measures angular data that are bimodal, based on a two-component circular-circular regression model with parameters that change according to a function expressed in a finite circular B-spline basis (see Section 7.2.1).

\subsubsection{Circular-linear regression}

Circular-linear regression is used to model the relationship between a circular response variable, $\Theta$, and a vector containing one or more covariates denoted here by $\boldsymbol{X}$. Mardia and Jupp (1999, Section 11.3.2) refer to the use of the link-based models of Fisher and Lee (1992) in this context. It would appear that the most popular such link function has been $2 \tan ^{-1}$. For this choice, the conditional mean is

$$
\mathrm{E}(\Theta \mid \boldsymbol{X}=\boldsymbol{x})=2 \tan ^{-1}\left(\boldsymbol{\beta}^{\prime} \boldsymbol{x}\right),
$$

where $\boldsymbol{\beta}$ is a vector of regression coefficients. This link function maps the origin of $\mathbb{R}$ to the angle 0 , and the two extremes of $\mathbb{R}$ to the angle furthest from 0 , namely $-\pi \equiv \pi$. Presnell et al. (1998) identified important practical difficulties with estimating the parameters of such models using ML methods. As a means of circumventing those inherent inferential problems, George and Ghosh (2006) proposed a semi-parametric Bayesian approach. Artes (2008) developed analysis of covariance tests for link-based models.

Instead of using link functions, Presnell et al. (1998) proposed an alternative modelling approach based on projecting (see Section 3.1) the unobserved responses from a multivariate linear model onto $\mathbb{S}^{1}$. This approach has become increasingly popular, particularly in Bayesian applications (see, e.g., Núñez-Antonio et al., 2011; Wang and Gelfand, 2013; Hernandez-Stumpfhauser et al., 2016). The interpretation of predictor effects in projected normal regression models has been considered recently by Cremers et al. (2018).

A different tack was taken by Lund (2002), who evaded the problem of devising a meaningful regression function through the use of a tree-based approach to predicting a circular response from a combination of circular and linear predictors.

Various approaches to modelling longitudinal data have been developed recently. Artes et al. (2000) considered the use of estimating equations when the angular response is assumed to follow a circular 
distribution parametrised by its mean direction and mean resultant length. D'Elia (2001) proposed a variance components model with fixed and random effects. Lagona (2016) introduced a regression model for correlated circular data which assumes that the angular measurements arise from the sine multivariate vM distribution of Mardia et al. (2008). All three of these proposals make use of the link function approach of Fisher and Lee (1992). The model of Lagona (2016) was extended by Mulder and Klugkist (2017), who employed weakly informative priors within a Bayesian framework to elude the problems with ML estimation for link-based models. Other researchers have adapted projected normal models. Núñez-Antonio and Gutiérrez-Peña (2014) investigated one in which the components are specified as mixed linear models. Maruotti (2016) considered a mixed linear model with correlated random coefficients controlling dependence that can be represented as a finite mixture of projected normal distributions. Maruotti et al. (2016) proposed a time-dependent extension of the projected normal regression model with a hidden Markov heterogeneity structure.

Many of the above proposals have been used to model animal orientation data. Other models for such data include that of Rivest et al. (2016), which features a consensus model for the angular response, based on circular and linear covariates, combined with vM errors. Recently, Rivest and Kato (2019) proposed a random effects circular regression model for clustered circular data where both the cluster effects and the regression errors have vM distributions. Their model is based on the multivariate angular pdf with vM-distributed cluster-level random effects of Holmquist and Gustafsson (2017). Other approaches to modelling animal orientation data are considered in Section 11.

\subsubsection{Linear-circular regression}

Linear-circular regression is applied to model the relationship between a linear response variable and one or more circular covariates. The standard approach is to regress the linear variable on sums of trigonometric polynomials of the circular variables, using least squares to estimate the parameters (Johnson and Wehrly, 1978). Bhattacharya and SenGupta (2009a) and SenGupta and Bhattacharya (2015) have considered Bayesian approaches to linear-circular modelling.

Recently, Cremers et al. (2020) proposed several regression models for a cylindrical response variable with linear and circular components.

\subsubsection{Spherical response}

Spherical regression was first considered by Chang (1986). Downs (2003) made use of Möbius transformation, stereographic projection and link functions to develop $\mathbb{S}^{2}-\mathbb{S}^{2}$ regression models with the conditional distribution between response and predictor being vMF. Hinkle et al. (2014) proposed polynomial models for manifold-linear regression. For $\mathbb{S}^{d}-\mathbb{S}^{d}$ regression, Rosenthal et al. (2014) employed projective linear transformations to model the conditional mean direction of the response, combined with a vMF error structure. Cornea et al. (2017) proposed a more general semi-parametric intrinsic manifold-manifold regression model that incorporates parametric link functions and a nonparametric error structure. Very recently, Paine et al. (2020) introduced a very general regression model for an $\mathbb{S}^{2}$-valued response with covariates that can be spherical, linear or categorical, and two kinds of anisotropic error distributions. In its most general formulation, a preliminary orthogonal transformation of the response is assumed to follow an anisotropic distribution with covariate-dependent parameters. For $\mathbb{S}^{d}-\mathbb{R}^{q}$ regression, Scealy and Wood (2019) proposed a flexible heteroscedastic model with scaled vMF errors.

Related regression problems for a $\mathbb{S}^{2}$-valued response include the fitting of small circles to spherical data (Rivest, 1999) and the analysis of rotational deformations through fitting small circles on the sphere (Schulz et al., 2015). 


\section{Nonparametric curve estimation}

Here we review advances in nonparametric curve estimation. See Section 5 for nonparametric tests, and later sections for other nonparametric methods.

\subsection{Density estimation}

\subsubsection{Smoothing-based}

Kernel density estimation (KDE) on $\mathbb{S}^{d}$ dates back to Beran (1979), Hall et al. (1987), and Bai et al. (1988). In the latter's formulation, the kernel estimator for a sample $\boldsymbol{X}_{1}, \ldots, \boldsymbol{X}_{n}$ from the target pdf $f$, is given by

$$
\hat{f}(\boldsymbol{x} ; h)=\frac{c_{L}(h)}{n} \sum_{j=1}^{n} L\left(\frac{1-\boldsymbol{x}^{\prime} \boldsymbol{X}_{j}}{h^{2}}\right), \quad \boldsymbol{x} \in \mathbb{S}^{d},
$$

where $h>0$ denotes the bandwidth, $L:[0, \infty) \rightarrow[0, \infty)$ a kernel, and $c_{L}(h)$ a normalising constant. For the vMF kernel $L(r)=e^{-r}$ and $d=1,(17)$ reduces to (6) with common concentration $\kappa=1 / h^{2}$, namely

$$
\hat{f}(\theta ; \kappa)=\frac{1}{2 \pi \mathcal{I}_{0}(\kappa) n} \sum_{j=1}^{n} \exp \left\{\kappa \cos \left(\theta-\Theta_{j}\right)\right\}, \quad \theta \in[-\pi, \pi) .
$$

Several extensions and modifications of (17) and (18) have been proposed. For $d \geq 2$, Klemelä (2000) used $L\left(\kappa \cos ^{-1}\left(\boldsymbol{x}^{\prime} \boldsymbol{X}_{j}\right)\right)$ in (17) to analyse estimators of $f$ and its derivatives. Extending (18) to $[-\pi, \pi)^{d}$, Di Marzio et al. (2011) introduced a class of Fourier-based sine-order circular kernels containing many well-known circular pdfs. García-Portugués et al. (2013b) considered the extension of $(17)$ to $\mathbb{S}^{d} \times \mathbb{R}$. Amiri et al. (2017) transformed (17) into a sequentially updating estimator. Tsuruta and Sagae (2017a) showed that using a WC kernel instead of a vM kernel in (18) worsens the optimal asymptotic mean integrated squared error (AMISE) rate from $n^{-4 / 5}$ to $n^{-2 / 3}$, despite both kernels being second sine-order. This motivated Tsuruta and Sagae (2017b) to propose a class of $p$-th order kernels with an optimal AMISE rate of $n^{-2 p /(2 p+1)}$.

Bandwidth selection is crucial to KDE and hence was also addressed in most of the aforementioned contributions. Plug-in selectors as alternatives to cross-validation (CV) have received most attention. Taylor (2008) proposed the first plug-in selector for (18) by deriving the AMISE under the assumption that $f$ is vM. The plug-in rule of Oliveira et al. (2012) employed the AMISE of Di Marzio et al. (2011), but used a two-component vM mixture in its curvature term. García-Portugués (2013) gave plug-in selectors for (17) using the AMISE and MISE for mixtures of vMF pdfs. Recently, Tsuruta and Sagae (2020) studied the convergence rates of direct plug-in and CV selectors for KDE on $\mathbb{S}^{1}$. Pham Ngoc (2019) proposed a bandwidth selector for (17) with a convergence rate of $n^{-2 p /(2 p+d)}$ for $p$-th order kernels.

Asymptotic results obtained for (17) include: CLTs for the integrated squared error of KDEs on $\mathbb{S}^{d}$ (Zhao and Wu, 2001), and $\mathbb{S}^{d} \times \mathbb{R}$ and $\mathbb{S}^{d_{1}} \times \mathbb{S}^{d_{2}}$ (García-Portugués et al., 2015); lower bounds for asymptotic minimax risks (Klemelä, 2003); laws for the iterated logarithm (Wang and Zhao, 2001, 2003); large and moderate deviations (Gao and Li, 2010; Li, 2014).

Convolutions on $\mathbb{S}^{d}$ are intimately related with $\mathrm{KDE}$ and are key to fast computation. They have been studied in Ĕgecioğlu and Srinivasan (2000), Dokmanic and Petrinovic (2010), and Le Bihan et al. (2016). 
As alternatives to (17) and (18), Wang and Ma (2000) introduced a nearest-neighbour estimator of $f$ and Park $(2012,2013)$ considered KDE via the tangent space of $\mathbb{S}^{d}$. Di Marzio et al. (2017) matched trigonometric moments of $f$ with their smoothed sample versions to derive pdf estimators. As in high-order KDE, such estimators lower the bias and retain the variance order of (18), although negative values are possible. Using a different approach, Di Marzio et al. (2016b, 2018a) investigated local likelihood (Loader, 1996) for pdfs on $[-\pi, \pi)^{d}$ by using local approximation of $\log f$. KDE based on the heat kernel on $\mathbb{S}^{d}$ (see Hartman and Watson, 1974), the $d=1$ case of which being the wrapped normal kernel, was applied in Zhang et al. (2019).

Extensions of (18) enable the construction of smoothed estimators for circular cdfs (Di Marzio et al., 2012b) and conditional pdfs (Di Marzio et al., 2016a). More generally, KDE has also been developed for compact Riemannian manifolds (Pelletier, 2005; Henry and Rodriguez, 2009), with inherent reduced tractability.

\subsubsection{Series-based}

An alternative approach to estimating a circular pdf is to use sample trigonometric moments as estimates of coefficients in its Fourier series expansion. Such estimates generally exhibit harmonic peaks and troughs and can be negative, although the latter defect can be circumvented by imposing constraints (Fernández-Durán, 2004). Instead, periodic Bernstein polynomials might be considered. However, as Carnicero et al. (2018) have shown, imposing periodicity on such polynomials increases the error rate from $n^{-4 / 5}$ to $n^{-2 / 3}$. An interesting connection between Fourier-based estimation and (18) arises through the use of the WC kernel (Chaubey, 2018).

Spherical harmonics (see, e.g., Dai and $\mathrm{Xu}, 2013$ ) extend Fourier orthogonal bases to $\mathbb{S}^{d}$ with increasing complexity as $d$ grows. Hence, pdf estimation through spherical harmonics inherits both the advantages and disadvantages of Fourier series estimation on the circle. A compelling alternative, are needlets (Narcowich et al. (2006); see also Marinucci et al. (2008)), a class of spherical wavelets. Needlets build on spherical harmonics to form a tight frame on $L^{2}\left(\mathbb{S}^{d}\right)$ that is not a basis, as redundancy is allowed, but has superior localisation properties. Needlet coefficients can be estimated from sample spherical harmonic coefficients. Baldi et al. (2009a) approached adaptive pdf estimation on $\mathbb{S}^{d}$ by thresholding needlet coefficients, and Kueh (2012) studied the latter estimator under varying local pdf regularity. Like Fourier-based estimates, needlet-based pdf estimates can take negative values.

Circular deconvolution, i.e. the estimation of a pdf on $\mathbb{S}^{1}$ from noisy observations (see Section 5.1), has been tackled with increasing generality in Efromovich (1997), Comte and Taupin (2003), and Johannes and Schwarz (2013). Spherical deconvolution has been studied through spherical harmonics (Healy et al., 1998; Kim and Koo, 2002; Kim et al., 2004) and needlets (Kerkyacharian et al., 2011).

\subsubsection{Bayesian-based}

Density estimation using Dirichlet process mixtures (DPMs) is a popular nonparametric Bayesian approach and has been employed with directional variables too. Lennox et al. (2009) provided a DPM model having sine bivariate vM distributions to model pairs of dihedral angles on $\mathbb{T}^{2}$. Straub et al. (2015) proposed a DPM model of Gaussian distributions in distinct tangent spaces to $\mathbb{S}^{d}$. DPM models with projected normal distributions have been advocated by Núñez-Antonio et al. (2018) on $\mathbb{S}^{1}$ and by Abraham et al. (2019) on $\mathbb{T}^{d}$. Density estimation through DPM on manifolds was addressed in Bhattacharya and Bhattacharya (2012, Chapter 13). 


\subsection{Regression estimation}

\subsubsection{Linear response}

The Nadaraya-Watson estimator for linear-spherical regression is

$$
\hat{m}(\boldsymbol{x} ; h)=\frac{c_{L}(h)}{n \hat{f}(\boldsymbol{x} ; h)} \sum_{j=1}^{n} Y_{j} L\left(\frac{1-\boldsymbol{x}^{\prime} \boldsymbol{X}_{j}}{h^{2}}\right), \quad \boldsymbol{x} \in \mathbb{S}^{d},
$$

which, for the vMF kernel and $\mathbb{S}^{1}$, reduces to

$$
\hat{m}(\theta ; \kappa)=\frac{\sum_{j=1}^{n} Y_{j} \exp \left\{\kappa \cos \left(\theta-\Theta_{j}\right)\right\}}{\sum_{j=1}^{n} \exp \left\{\kappa \cos \left(\theta-\Theta_{j}\right)\right\}}, \quad \theta \in[-\pi, \pi) .
$$

As an extension of (20), Di Marzio et al. (2009) introduced local polynomial regression for predictors on $\mathbb{T}^{d}$ through a sine term-based Taylor expansion. Their approach was extended further by Qin et al. (2011) to accommodate circular and multivariate predictors using product kernels, a broadly applicable approach to combine different predictors. Tsuruta and Sagae (2018) showed the different optimal error rates for the (second sine-order) WC and vM kernels.

On $\mathbb{S}^{d}$, (19) was considered by Wang et al. (2000) and Wang (2002) when deriving laws for iterated logarithm and exponential error bounds, respectively. Di Marzio et al. (2014) extended (20) to local polynomial regression using a Taylor expansion within the tangent-normal decomposition. García-Portugués et al. (2016) used a different Taylor expansion yielding a local linear estimator that, for $d=1$, coincides with the Di Marzio et al. (2009) proposal. Di Marzio et al. (2019b) built on their construction in Di Marzio et al. (2014) to perform local polynomial logistic regression with a spherical predictor.

Monnier (2011) proposed needlet-based regression for a uniformly distributed predictor on $\mathbb{S}^{d}$ and Gaussian noise, while Lin (2019) weakened those assumptions and introduced regularisation on the needlet coefficients.

Thin-plate splines on $\mathbb{S}^{d}$ (Taijeron et al., 1994) offer an alternative smoothing approach to kernel methods. Such splines have been considered for improving brain conformal mapping to $\mathbb{S}^{2}$ (Zou et al., 2007). Kaufman et al. (2005) introduced circular Bayesian adaptive regression splines for modelling the firing rates of neurons activated by movements of a monkey's wrist. Quadratic Bsplines on the circle were constructed in McMillan et al. (2013).

Related to regression for a $\mathbb{S}^{1}$ predictor, Hall et al. (2000), Hall and Yin (2003), and Genton and Hall (2007) studied the estimation of periodic functions over an (unwrapped) time domain. Klemelä (1999) considered the estimation of a function on $\mathbb{S}^{d}$ observed in Gaussian continuous time white noise.

\subsubsection{Circular or spherical response}

Boente and Fraiman (1991) considered estimators for $\mathbb{S}^{d}-\mathbb{R}^{q}$ regression based on locally-weighted spherical means, with nearest-neighbour or Nadaraya-Watson weights. Their construction was generalised to local polynomial $\mathbb{S}^{1}-\mathbb{S}^{1}$ and $\mathbb{S}^{1}-\mathbb{R}$ (Di Marzio et al., 2013), $\mathbb{S}^{1}-\mathbb{R}^{q}$ (Meilán-Vila et al., 2020), and $\mathbb{S}^{d}-\mathbb{S}^{q}$ (Di Marzio et al., 2014) regression through local circular and spherical means. A novel approach to $\mathbb{S}^{d}-\mathbb{S}^{d}$ regression, based on local polynomial expansions of the rotation function, was advocated by Di Marzio et al. (2019c). 
Quantile $\mathbb{S}^{1}$-S $\mathbb{S}^{1}$ and $\mathbb{S}^{1}-\mathbb{R}$ regression was developed by Di Marzio et al. (2016c) through inversion of the conditional circular distribution and smoothing a circular check function.

From a Bayesian perspective, Scott (2011) estimated the regression function on $\mathbb{S}^{2}$ by imposing shrinkage priors on its needlet coefficients. Navarro et al. (2017) proposed multivariate generalized vM circular processes as a replacement for Gaussian processes in circular regression.

More generally, Cheng and Wu (2013) addressed linear-manifold regression through local linear regression on the tangent space, and Lin et al. (2017) gave an extrinsic Nadaraya-Watson estimator for manifold-linear regression.

\section{Dimension reduction methods}

\subsection{Principal component analysis}

\subsubsection{General manifolds}

Principal component analysis (PCA) for data on a Riemannian manifold $\mathcal{M}$ of dimension $d$, such as $\mathbb{S}^{d}$ or $\mathbb{T}^{d}$, has received considerable attention lately. Approaches to manifold PCA can be classified using two broad dichotomies: (i) extrinsic (based on tangent space) versus intrinsic (geodesic-based); (ii) forward (sequential computation of the $j$-th principal component, $j=1, \ldots, d$ ) versus backward (computation of a sequence of nested subspaces of decreasing dimension within $\mathcal{M}$ based on constraints; Damon and Marron (2014)). Huckemann et al. (2010) gave a detailed review of the topic, and Marron and Alonso (2014) and Pennec (2018) more recent overviews.

Fletcher et al. (2004) introduced principal geodesic analysis (PGA) as an analogue of PCA in symmetric spaces such as $\mathbb{S}^{d}$ and $\mathbb{T}^{d}$. It is centred upon the intrinsic sample mean on $\mathcal{M}, \hat{\boldsymbol{\mu}}$, and defines the first principal geodesic as the one passing through $\hat{\boldsymbol{\mu}}$ that minimises the sum of squared intrinsic residuals. Other principal geodesics are obtained sequentially by imposing orthogonality at $\hat{\boldsymbol{\mu}}$. PGA involves a complex optimisation process, only solved later by Sommer et al. (2014). This complexity led Fletcher et al. (2004) to propose tangent PCA (tPCA) as an approximation. tPCA performs PCA with the log-mapped data onto the tangent plane at $\hat{\boldsymbol{\mu}}$ and then obtains the principal geodesics on $\mathcal{M}$ spanned by the tangent principal directions. When $\mathcal{M}=\mathbb{S}^{2}$, the principal components of PGA and tPCA are great circles that pass through $\hat{\boldsymbol{\mu}}$.

Two limitations of PGA are exemplified on $\mathbb{S}^{2}$ : (a) great circles are forced to cross at $\hat{\boldsymbol{\mu}}$; (b) great circles are unable to describe certain forms of variation (see Section 8.1.2). Huckemann and Ziezold (2006) tackled (a) by introducing geodesic PCA (GPCA) for Riemannian manifolds, a forward-type method with a backward shift that locates a data centre $\tilde{\boldsymbol{\mu}}$ after finding the best fitting geodesic. The other components cross orthogonally at $\tilde{\boldsymbol{\mu}}$, a restriction circumvented by horizontal component analysis (Sommer, 2013). Curry et al. (2019) recently proposed principal symmetric space approximation (PSSA), which considers totally geodesic subspaces (great subspheres, on $\mathbb{S}^{d}$ ) and is computationally tractable on certain manifolds.

A non-geodesic approach to PCA on $\mathcal{M}$ is barycentric subspace analysis (Pennec, 2018). It considers $k$-dimensional affine spans (great subspheres if $\mathcal{M}=\mathbb{S}^{d}$ ) spanned by $k+1 \mathcal{M}$-affinely independent points, whose successive addition/removal yields a forward/backward-type sequence of nested subspaces.

Zhang and Fletcher (2013) proposed probabilistic PGA, in which the normal distribution used in probabilistic PCA (Tipping and Bishop, 1999) is replaced by what the authors refer to as the Rie- 
mannian normal distribution, with pdf $f\left(\boldsymbol{x} ; \boldsymbol{\mu}, \sigma^{2}\right) \propto \exp \left\{-d_{g}(\boldsymbol{x}, \boldsymbol{\mu})^{2} /\left(2 \sigma^{2}\right)\right\}$, where $\boldsymbol{x}, \boldsymbol{\mu} \in \mathcal{M}$ and $d_{g}$ is the intrinsic distance on $\mathcal{M}\left(d_{g}(\boldsymbol{x}, \boldsymbol{\mu})=\cos ^{-1}\left(\boldsymbol{x}^{\prime} \boldsymbol{\mu}\right)\right.$ if $\left.\mathcal{M}=\mathbb{S}^{d}\right)$. Sommer (2019) advocated an alternative to PGA based on an anisotropic normal distribution over $\mathcal{M}$, generated from the marginal distributions of a diffusion process on $\mathcal{M}$ with a constant infinitesimal covariance.

The previous approaches assume a parametric form for the first principal curve on $\mathcal{M}$. Instead, the principal flow of Panaretos et al. (2014) is defined as the curve of maximal data variation on $\mathcal{M}$ that, starting at $\hat{\boldsymbol{\mu}}$, is tangential to the vector field formed by the first eigenvector of the local tangent covariance matrix. Higher-order principal flows, which are always curves, are defined analogously.

Dai and Müller (2018) adapted tPCA for functional data on $\mathcal{M}$ (e.g., flight trajectories on $\mathbb{S}^{2}$ ) by replacing PCA by functional PCA on the tangent plane.

Nonparametric inference on backward nested principal component subspaces, generalising the result of Anderson (1963) on asymptotic inference for classical PCA, has been provided by Huckemann and Eltzner (2018).

\subsubsection{Methods for spherical data}

In relation to limitation (b) of Section 8.1.1, and for the specific case of $\mathbb{S}^{2}$, Jung et al. (2011) advocated principal arc analysis (PAA), a non-geodesic approach designed to improve the flexibility of GPCA. PAA employs small circles on $\mathbb{S}^{2}$ as the primary modes of data variation, an idea generalised to $\mathbb{S}^{d}$ by Jung et al. (2012) as principal nested spheres (PNS). By iteratively performing a series of tangent-normal decompositions on $\mathbb{S}^{d}$, PNS is a backward-type approach that produces a sequence of subspheres isomorphic to $\mathbb{S}^{j}, j=d-1, \ldots, 1$, that none of the methods in Section 8.1.1 are able to match in terms of flexibility.

Despite the generality of the approaches in Section 8.1.1, the success of PNS highlights the advantages of focusing on specific manifolds, such as $\mathbb{S}^{d}$ or $\mathbb{T}^{d}$, and exploiting their peculiarities so as to obtain more informative methods. Other examples of the benefits of specificity include PAA on direct product manifolds such as $\left(\mathbb{R}^{3} \times \mathbb{R}^{+} \times \mathbb{S}^{2} \times \mathbb{S}^{2}\right)^{m}$, introduced by Jung et al. (2011), and composite PNS for skeletal models, proposed by Pizer et al. (2013).

\subsubsection{Methods for toroidal data}

Torus-specific PCA proposals have been stimulated by the need to analyse dihedral angles in bioinformatics, and the inapplicability of most of the methods in Section 8.1.1 due to the pathological behaviour of geodesics on $\mathbb{T}^{d}$.

The majority of toroidal PCA methods resort to some sort of transformation prior to applying classical PCA. Mu et al. (2005) proposed dihedral PCA (dPCA) by mapping angles from $[-\pi, \pi)^{d}$ to $\mathbb{T}^{d}$ and then performing PCA. Complex dPCA (Altis et al., 2007) performs PCA on the complex representation of angles. Riccardi et al. (2009) proposed angular PCA (aPCA), based on applying PCA to toroidal data centred on their circular means. Kent and Mardia (2009) gave a trigonometric moment characterisation of the covariance matrix in a wrapped normal model on $\mathbb{T}^{d}$, facilitating PCA on it. Nodehi et al. (2015) applied PGA on $\mathbb{T}^{d}$ in what they called dPGA. The latter two approaches yield principal directions that almost surely wrap around infinitely. With regard to this issue, Kent and Mardia (2015) discussed desirable properties for principal component curves on $[-\pi, \pi)^{2}$. Sittel et al. (2017) introduced a variation on aPCA, called $\mathrm{dPCA}+$, that shifts each variable so that $-\pi \equiv \pi$ is located at the lowest pdf region for minimising the distortion when PCA is applied. Sargsyan et al. $(2012,2015)$ used a non-injective mapping from $\mathbb{T}^{d}$ to $\mathbb{S}^{d}$ that equates 
toroidal angles in $[-\pi, \pi)^{d}$ to hyperspherical coordinate angles, even though the latter are defined in $[0, \pi]^{d-1} \times[-\pi, \pi)$, then applied PGA.

A better-grounded approach to torus PCA is T-PCA (Eltzner et al., 2018), which maps $\mathbb{T}^{d}$ to $\mathbb{S}^{d}$ with a deformation, which, for $d=2$, corresponds to cutting $\mathbb{T}^{2}$ at a data-driven point to form a cylinder, contracting the circles at its ends to single points, and reconnecting at those points. Principal nested deformed spheres (Eltzner et al., 2015) is an extension of the T-PCA approach to data on a polysphere $\mathbb{S}^{d_{1}} \times \cdots \times \mathbb{S}^{d_{m}}$. PSSA can also be applied to toroidal data, with geodesics on $\mathbb{T}^{d}$ identified using model selection.

\subsection{Other dimension reduction methods}

Nonlinear dimension reduction methods for Euclidean data have also been adapted to the directional context. Lunga and Ersoy (2013) modified $t$-stochastic neighbour embedding ( $t$-SNE) of van der Maaten and Hinton (2008) to obtain a dimension reduction method, from $\mathbb{S}^{d}$ to $\mathbb{S}^{q}, q \ll d$, using neighbourhoods formed by the extension of the WC distribution to $\mathbb{S}^{d}$ in Kato (2009). Wang and Wang (2016) proposed a modification of $t$-SNE with vMF neighbourhoods.

Wilson et al. (2014) revisited multidimensional scaling on $\mathbb{S}^{d}$, proposing a new approach that obviated the minimisation of stress functions based on spherical distances inherent in former approaches. They used their approach to map textures of 3D objects onto spheres (Elad et al., 2005), and model normalised time-warping functions (Veeraraghavan et al., 2009). Lu et al. (2019) adapted $t$-SNE for dimension reduction from $\mathbb{R}^{d}$ to $\mathbb{S}^{q}, q \ll d$, highlighting the benefits of the clusterings obtained on the spherical geometry. Note that these transformations of multivariate data into spherical data, termed spherical embeddings, call for the use of directional statistics with data which were not originally directions.

\section{Classification and clustering}

\subsection{Classification}

SenGupta and Roy (2005) introduced a discrimination rule based on the chordal distance between a new circular observation and those from two known circular populations. More recently, Di Marzio et al. (2018b) considered nonparametric circular classification based on KDE and local logistic regression. Pandolfo et al. (2018a) studied the depth-versus-depth classifier for circular data. Leguey et al. (2019a) proposed Bayesian classification algorithms for WC-distributed circular predictors.

SenGupta and Ugwuowo (2011) proposed generalised likelihood-ratio tests for classifying toroidal and cylindrical data into two populations when one of the misclassification probabilities is assumed to be known. Fernandes and Cardoso (2016) developed a logistic classifier for use with circular and linear predictors.

Classification rules for data on $\mathbb{S}^{d}$ from Watson and vMF populations were developed by Figueiredo and Gomes (2006) and Figueiredo (2009), respectively. Bhattacharya and Dunson (2012) proposed a Dirichlet process mixture model classifier comprising vMF kernels. López-Cruz et al. (2015) considered the naive Bayes classifier with vMF-related conditional distributions of directional predictors. Techniques for the classification of image textures, based on multi-resolution directional filters, were proposed by Kim and So (2018). Di Marzio et al. (2019a) considered KDE-based nonparametric classification. The cosine depth distribution classifier was introduced in Demni et al. (2019). A comparison of different classification rules on $\mathbb{S}^{2}$ was performed by Tsagris and Alenazi (2019). 


\subsection{Clustering}

The development of clustering methods for directional data has been a major research theme lately, particularly amongst the machine learning community.

The two most popular approaches to clustering data on $\mathbb{S}^{d}$ are spherical $k$-means and the use of (finite) mixture models with vMF components, the vMF extension of (6). Both have often been applied after projecting data in $\mathbb{R}^{d+1}$ to $\mathbb{S}^{d}$. The spherical $k$-means approach (Dhillon and Modha, 2001) maximises the cosine similarity measure $\sum_{j=1}^{n} \boldsymbol{X}_{j}^{\prime} \boldsymbol{c}_{(j)}$ between the sample $\boldsymbol{X}_{1}, \ldots, \boldsymbol{X}_{n}$ and $k$ centroids $\boldsymbol{c}_{1}, \ldots, \boldsymbol{c}_{k} \in \mathbb{S}^{d}$, where $\boldsymbol{c}_{(j)}$ is the centroid of the cluster containing $\boldsymbol{X}_{j}$.

Dhillon and Sra (2003) and Banerjee et al. (2003, 2005) gave Expectation-Maximisation (EM) algorithms for fitting vMF mixtures. Such mixtures accommodate spherical $k$-means as a particular limiting case (Banerjee et al., 2005). Other approaches to fitting vMF mixtures include those of: Yang and Pan (1997), based on embedding fuzzy partitions in the mixtures; Taghia et al. (2014), a Bayesian approach employing variational inference; Gopal and Yang (2014), who proposed Bayesian graphical modelling approaches based on variational inference and collapsed Gibbs sampling; Qiu et al. (2015), which used a new information criterion to determine the number of clusters; Kasarapu and Allison (2015), based on the Bayesian minimum message length criterion to determine the optimal number of components; Mash'al and Hosseini (2015), a $k$-means ++ method for identifying favourable mixture starting values; Salah and Nadif (2019), a co-clustering approach based on diagonal block mixtures of vMF distributions. Mixtures with vMF components have been employed to model data from photometry (Hara et al., 2008), text mining (Banerjee et al., 2009), speech recognition (Tang et al., 2009), radiation therapy (Bangert et al., 2010), pattern recognition (Calderara et al., 2011), multichannel array processing (Costa et al., 2014), and collaborative filtering (Salah and Nadif, 2017). vMF mixtures have been used to cluster pdf objects based on their sphericalvalued wavelet coefficients (Montanari and Calò, 2013).

Clustering approaches based on mixtures with other types of directional distributions have also been advocated. To increase cluster shape modelling flexibility on $\mathbb{S}^{d}$, Peel et al. (2001) used mixtures of Kent distributions, whereas Dortet-Bernadet and Wicker (2008) proposed ones with inverse stereographic projections of multivariate normal distributions. Mixture models with wrapped normal components were investigated by Agiomyrgiannakis and Stylianou (2009), and used to cluster X-ray position data in Abraham et al. (2013). Bayesian approaches to fitting projected normal mixtures have been proposed by Wang and Gelfand (2014) and Rodríguez et al. (2020), and for general projected normal mixtures by Hernandez-Stumpfhauser et al. (2017). Franke et al. (2016) developed an EM algorithm to fit the latter type of mixtures to data on $\mathbb{S}^{2}$. For data on $\mathbb{T}^{d}$, Mardia et al. (2012) proposed mixtures of concentrated sine multivariate $\mathrm{vM}$ components, with approximated normalising constants, to cluster dihedral angles of an amino acid. For cylindrical data, Lagona and Picone (2011, 2012) developed latent-class mixture models to cluster incomplete environmental data.

Mixture model-based approaches for clustering axial data have also been proposed. Bijral et al. (2007), Souden et al. (2013), and Sra and Karp (2013) developed EM-based algorithms to fit mixtures of Watson distributions. Hasnat et al. (2014) provided an alternative approach to fitting such models based on Bregman divergence. A clustering approach based on mixtures of Bingham distributions was developed by Yamaji and Sato (2011).

Variations of spherical $k$-means include the spherical fuzzy and possibilistic $c$-means proposed by Kesemen et al. (2016) and Benjamin et al. (2019), respectively, and the adaptations by Maitra and Ramler (2010) for computational efficiency. On $\mathbb{S}^{1}$, Baragona (2003) further investigated an alternative partitioning based on the statistic of Lund (1999). A nonparametric alternative to $k$-means is 
kernel mean shift clustering, introduced on $\mathbb{S}^{d}$ by Oba et al. (2005). It was then extended by Cetingul and Vidal (2009) to $\mathbb{S}^{d}$ and other specific manifolds, using intrinsic and extrinsic perspectives, and later reintroduced on $\mathbb{S}^{d}$ with minor variations (Chang-Chien et al., 2010; Yang et al., 2014). A modification of kernel mean shift that uses time-varying bandwidths was adapted to spherical data by Hung et al. (2015).

\section{Modelling serial dependence}

\subsection{Discrete-time processes}

Let $\left\{\Theta_{t}\right\}_{t=1,2, \ldots}$ denote a discrete-time circular process, and $\left\{\theta_{t}\right\}_{t=1,2, \ldots, n}$ a corresponding circular time series. Mardia and Jupp (1999) provide a summary of the projected normal, wrapped, linked autoregressive moving average (ARMA), and circular autoregressive (CAR) models for circular time series considered in Fisher and Lee (1994) and Fisher (1993, Section 7.2).

If $\left\{\left(X_{t}, Y_{t}\right)\right\}_{t=1,2, \ldots}$ is a stationary bivariate normal process then $\left\{\Theta_{t}\right\}_{t=1,2, \ldots}$, where $\Theta_{t}=\operatorname{Arg}\left(X_{t}+\right.$ $\left.\mathrm{i} Y_{t}\right)$, is a projected normal process. If $\left\{X_{t}\right\}_{t=1,2, \ldots}$ is a process on $\mathbb{R}$ then $\left\{\Theta_{t}\right\}_{t=1,2, \ldots}$, where $\Theta_{t}=$ $X_{t}(\bmod 2 \pi)$, is the corresponding wrapped process. The wrapped AR processes of Breckling (1989) provide an example. A linked process is defined through $\Theta_{t}=\mu+g\left(X_{t}\right)$ where $\mu \in[-\pi, \pi)$ and $g$ is a link function defined in Fisher (1993, Section 7.2.4) as a mapping from $\mathbb{R}$ to $(-\pi, \pi)$. A linked $\operatorname{ARMA}(p, q)$ process is obtained if $X_{t}=g^{-1}\left(\Theta_{t}\right)$ is an $\operatorname{ARMA}(p, q)$ process. A CAR process is one for which $\Theta_{t} \mid \Theta_{t-1}=\theta_{t-1}, \ldots, \Theta_{t-p}=\theta_{t-p}$ is vM-distributed with mean direction

$$
\mu_{t}=\mu+g\left[\alpha_{1} g^{-1}\left(\theta_{t-1}-\mu\right)+\cdots+\alpha_{p} g^{-1}\left(\theta_{t-p}-\mu\right)\right]
$$

and concentration parameter $\kappa$, where $\mu \in[-\pi, \pi)$ and $\alpha_{1}, \ldots, \alpha_{p} \in \mathbb{R}$. Artes and Toloi (2009) proposed an extension of the CAR model with covariates. Processes with distributions other than the vM can be defined analogously to CAR. Erdem and Shi (2011) consider four ARMA-based approaches to the short-term forecasting of wind speed and direction.

Markov models can be constructed using a transition pdf, $f_{\Theta_{t} \mid \Theta_{t-1}=\theta_{t-1}}$, derived from a bivariate circular pdf. Hughes (2007) used this approach, dating back to Wehrly and Johnson (1980), to obtain stationary Markov processes from the sine and cosine bivariate vM models referred to in Section 3.3, and the Möbius transformation-based regression model of Downs and Mardia (2002). Similarly, Kato (2010) employed the regression model of Kato et al. (2008) to derive a stationary Markov process. Yeh et al. (2013) proposed a circular Markov process based on a transition pdf that belongs to the class of generalized vM distributions referred to in Section 3.1. Le Bihan et al. (2016) studied Markov processes with rotationally symmetric transition pdfs on $\mathbb{S}^{d}$, specifically analysing the vMF case.

In a paper that has stimulated much research into the modelling of time series, spatial and spatiotemporal data (see Section 11), Holzmann et al. (2006) introduced hidden Markov models (HMMs) (Zucchini et al., 2016) for circular as well as cylindrical time series. Such models offer considerable flexibility in their serial dependence properties and use mixtures of varying distributions to model different underlying regimes. More specifically, Holzmann et al. (2006) considered circular HMMs with state-dependent vM, wrapped normal or WC distributions, and marginal distributions that are mixtures of each. They also proposed a cylindrical HMM with state-dependent vM distributions for the circular component. Bulla et al. (2012) extended this approach to develop a multivariate hidden Markov model for bivariate circular and bivariate linear data with sine bivariate vM and bivariate skew-normal pdfs. An HMM for toroidal time series using sine bivariate vM pdfs and allowing for missing observations was proposed in Lagona and Picone (2013). Hokimoto and Shimizu 
(2014) developed a model incorporating a non-homogeneous HMM with cylindrical covariates. Ailliot et al. (2015) proposed Markov-switching autoregressive models based on a non-homogeneous hidden Markov chain for circular time series with vM innovations. HMMs for use with cylindrical time series have been proposed by Lagona et al. (2015a), Mastrantonio et al. (2015), and Mastrantonio and Calise (2016). Mastrantonio et al. (2015) considered a projected normal-based extension of the model of Bulla et al. (2012) that allows for conditional correlation between the circular and linear variables. The Dirichlet process mixture model of Mastrantonio and Calise (2016) is designed for use with discrete cylindrical variables.

HMMs with toroidal components have also been employed in protein structure modelling. Boomsma et al. (2008) proposed one with cosine bivariate vM distributions to model the pairs of dihedral angles describing protein backbones. Lennox et al. (2010) considered a Dirichlet process mixture of HMMs with sine bivariate vM distributions for the dihedral angles. Golden et al. (2017) developed an HMM to model the evolution of pairs of proteins, with bivariate wrapped normal diffusions (García-Portugués et al., 2019) used to describe dihedral angle evolution.

Recently, Mazumder and Bhattacharya (2017) proposed a state-space model for circular time series, with a circular latent process, based on wrapped Gaussian processes (Mazumder and Bhattacharya, 2016). Beran and Ghosh (2020) introduced a class of linked processes for circular time series, allowing for long-range dependence, obtained by transforming Gaussian processes. Hokimoto and Shimizu (2008) extended the multiple regression model of Johnson and Wehrly (1978) to develop a time series model for data on $\mathbb{T}^{d} \times \mathbb{R}^{q}$.

Nonparametric kernel-based trend estimation in circular time series was tackled in Di Marzio et al. (2012a). Beran (2016) proposed a class of nonparametric normalised symmetric linear estimators for the trend of $\mathbb{S}^{d}$-valued time series.

\subsection{Continuous-time processes}

Continuous-time processes involving directional data have received considerably less attention than discrete-time processes. Hill and Häder (1997) introduced a random walk model whose reorientation process follows a vM diffusion (Kent, 1975). Several variations of such random walk models have been developed for biological purposes: see Codling et al. (2008) for a review. García-Portugués et al. (2019) proposed various Langevin diffusions on the torus that can be viewed as analogues of Ornstein-Uhlenbeck processes, and studied likelihood-based estimation approaches for them. Sommer (2019) considered anisotropic diffusion processes on Riemannian manifolds, and Jensen et al. (2019) simulated diffusion bridges on $\mathbb{T}^{d}$. Ball et al. (2008) introduced Brownian motion and Ornstein-Uhlenbeck processes on the shape space of $\mathbb{R}^{2}$.

Kurz et al. (2019) provided an overview of various recursive filtering algorithms involving a variety of circular, toroidal, and spherical distributions. Analogues of the Kalman filter based on the vMF and Bingham distributions on $\mathbb{S}^{d}$ were proposed by Chiuso and Picci (1998) and Kurz et al. (2014), respectively. Filtering using the wrapped normal distribution on $\mathbb{S}^{1}$ was considered by Traa and Smaragdis (2013). Pitt and Shephard (1999) proposed auxiliary particle filter methods and applied them to a ship tracking problem modelled using a WC process.

\section{Spatial and spatio-temporal modelling}

The modelling of spatial and space-time directional data is one of the branches of directional statistics that has experienced particularly important advances in recent years. Many contributions involve fitting hierarchical Bayesian spatial models to meteorological data using MCMC methods. As an 
approach to modelling hurricane winds, Modlin et al. (2012) proposed a Bayesian hierarchical model for vector fields featuring a wrapped normal conditional autoregressive model. Jona-Lasinio et al. (2012) formulated a similar model incorporating, instead, a wrapped Gaussian spatial process to model wave directions at different sea locations. Using a different perspective, Wang (2013), Wang and Gelfand (2014), and Wang et al. (2015) considered models based on projected normal processes for modelling wave direction and height at different sites.

As Lagona et al. (2015b) has pointed out, such Bayesian hierarchical models require specific assumptions on the prior distributions of the parameters of interest and ad-hoc MCMC for fitting. Instead, Lagona et al. (2015b) developed an HMM to model the temporal evolution of the sea surface in terms of time-varying circular-linear patterns that arise through latent environmental conditions. Fitting is performed using a pseudo-likelihood approach.

Extending the wrapped normal-based Bayesian approach of Jona-Lasinio et al. (2012), Mastrantonio et al. (2016a) introduced a wrapped skew-normal process, for use with spatio-temporal circular data, which is capable of modelling asymmetric marginal distributions. Mastrantonio et al. (2016b) extended and compared the processes of Jona-Lasinio et al. (2012) and Wang and Gelfand (2014) to the spatio-temporal setting by introducing space-time dependence and space- and time-varying covariate information.

In Lagona and Picone (2016) and Ranalli et al. (2018), hidden Markov random field models were proposed for the analysis of cylindrical spatial series, enabling segmentation of latent environmental conditions. Jona-Lasinio et al. (2018) and Lagona (2018) provided overviews of many of the developments discussed above. Ameijeiras-Alonso et al. (2019b) extended the approach of Ranalli et al. (2018) to develop a hidden Markov random field for the spatial segmentation of wildfires, using a mixture of Kato and Jones (2015) pdfs with parameters varying according to a latent nonhomogeneous Potts model.

Next we consider models for animal orientation data based on random walks and HMMs that provide alternatives to those in Section 6.2.2. Morales et al. (2004) proposed a Bayesian approach to fitting multiple random walks to animal movement data with paths composed of random step-lengths and turning angles. Each step and turn is assigned to a random walk characteristic of a hidden behavioural state. A similar approach was proposed by McClintock et al. (2012), with movement paths considered to be movement strategies between which animals switch in response to environmental factors. The authors combined a variety of methodologies to develop a suite of models based on biased and correlated random walks that allow for different forms of movement. Nicosia et al. (2017) proposed a hidden-state random walk model in which a circular-linear process models the direction and distance between consecutive positions of an animal, and the hidden states describe the animal's behaviour.

Random fields on $\mathbb{S}^{2}$ are discussed in depth in Marinucci and Peccati (2011). Amongst many other advances, their monograph analyses recent high-frequency limit results and tests for Gaussianity and isotropy of scalar-valued random fields, and considers applications in the analysis of the cosmic microwave background. Recent research into isotropic Gaussian random fields on $\mathbb{S}^{2}$ has developed CLTs for functionals of needlet coefficients (Baldi et al., 2009b), limit results for the first Minkowski functional (Leonenko and Ruiz-Medina, 2018), isotropy tests based on spherical harmonics (Sahoo et al., 2019), and tests for the detection of local maxima on isotropic fields (Cheng et al., 2020). The construction of valid covariance functions on $\mathbb{S}^{d}$, for use in geostatistics, has been summarised in the excellent overview of Gneiting (2013). New covariance functions on $\mathbb{S}^{d}$ include the spatio-temporal covariance functions of Porcu et al. (2016) and the matrix-valued covariance functions of Guella 
et al. (2018); see also the review by Porcu et al. (2020). A review of advances in the construction of covariance functions and process models on $\mathbb{S}^{2}$ was given in Jeong et al. (2017).

Irwin et al. (2002) gave a review of spatio-temporal nonlinear filtering and illustrated the use of cylindrical filtering in the analysis of battlespace data.

\section{Other topics}

\subsection{Statistical depth}

Agostinelli and Romanazzi (2013) studied, mainly for $\mathbb{S}^{1}$ and $\mathbb{S}^{2}$, the angular simplicial depth of Liu and Singh (1992) and the angular Tukey depth of Small (1987). Within the class of rotationally symmetric distributions on $\mathbb{S}^{d}$, Ley et al. (2014) defined a depth based on the quantiles of the sample projections onto the mean direction. Pandolfo et al. (2018b) introduced computationally tractable distance-based depths on $\mathbb{S}^{d}$, illustrating their use in location estimation and classification. A nonparametric approach to constructing tolerance regions for spherical data was proposed by Mushkudiani (2002).

\subsection{Design and analysis of experiments}

Otieno and Anderson-Cook (2012) provided an overview of the design of experiments involving directional variables, and methods available for analysing the data obtained from them. Recently, optimal designs for linear-spherical regression, based on Fourier series and spherical harmonics, have been established for $\mathbb{S}^{1}$ by Dette and Melas (2003), for $\mathbb{S}^{2}$ by Dette et al. (2005) and Dette and Wiens (2009), and for $\mathbb{S}^{d}$, with $d>2$, by Dette et al. (2019).

\subsection{Order-restricted analysis}

The random-periods model (RPM) of Liu et al. (2004) is a nonlinear regression model used to estimate the phase angles of periodically expressed genes. Rueda et al. (2009) developed circular isotonic regression estimation to infer the relative order of phase angles from the unconstrained estimates of the RPM. Fernández et al. (2012) proposed a test for a specified ordering of phase angles assuming the unconstrained estimators of the RPM to be vM-distributed. Barragán et al. (2015) developed methods for estimating and testing for a common ordering of phase angles across multiple experiments. A review of such developments was provided by Rueda et al. (2015). Subsequently, Rueda et al. (2016) proposed a piecewise circular regression model for the relationship between the phase angles of cell-cycle genes in two species with differing periods, and Barragán et al. (2017) considered the problem of aggregating different circular orders for the peak expressions of genes coming from heterogeneous datasets. Recently, Larriba et al. (2020) proposed a circular signal plus error model for identifying components of systems displaying rhythmic temporal patterns.

Independently of these developments, Klugkist et al. (2012), Baayen et al. (2012), and Baayen and Klugkist (2014) proposed ANOVA tests under order restrictions on the mean directions of vM distributions.

\subsection{Outlier detection}

New tests for detecting outliers in circular data, based on the circular distance (1), sums of such distances, and gaps, were introduced and compared with existing procedures in a series of papers referred to by Mahmood et al. (2017). Sau and Rodriguez (2018) developed a minimum distance approach to estimating the parameters of spherical models that provides an outlier detection tool. 
Outlier detection tests for cylindrical, simple circular regression, and circular time series data were proposed in Sadikon et al. (2019), Abuzaid et al. (2013), and Abuzaid et al. (2014), respectively.

Eigenvalue, likelihood-ratio, and geodesic distance-based tests for detecting outliers in axial data from an assumed underlying Watson distribution were developed in Figueiredo and Gomes (2005), Figueiredo (2007), and Barros et al. (2017).

\subsection{Compositional data analysis}

Compositional data analysis is used when the data under consideration are vectors of non-negative proportions summing to one. The most popular approach to analysing such data is that of Aitchison (1986). However, an alternative approach, based on the square-root transformation from a unit simplex to $\mathbb{S}^{d}$, was discussed in Stephens (1982). Recently, that approach has been further developed by Scealy and Welsh (2011, 2014a,b, 2017). The relationship between compositional and directional data was further exploited by Cuesta-Albertos et al. (2009) in the context of testing for uniformity.

\section{Software}

Historically, a major impediment to the application of directional statistics was a lack of software implementing the methodology particular to it. In recent years, the advent of the $\mathrm{R}$ statistical computing environment (R Core Team, 2020) and its ecosystem of contributed packages has partially addressed that paucity. An overview of many such packages was given by Pewsey (2018). Relevant packages written in other languages include CircStat (Berens, 2009) and PyCircStat (Sinz et al., 2018) for data on $\mathbb{S}^{1}$, libDirectional (Kurz et al., 2019) for data on $\mathbb{T}^{d}$ and $\mathbb{S}^{d}$, Mocapy++ for constructing probabilistic models of biomolecular structure (Paluszewski and Hamelryck, 2010), and the promising geomstats (Miolane et al., 2020) for manifold-valued data.

\subsection{General-purpose packages}

There are two main $\mathrm{R}$ packages designed for use with directional data: circular (Agostinelli and Lund, 2017) and Directional (Tsagris et al., 2020). Both include functions for the analysis of data on $\mathbb{S}^{1}, \mathbb{T}^{2}$, and $\mathbb{S}^{1} \times \mathbb{R}$. Directional also has routines for data on $\mathbb{S}^{d}$.

For data on $\mathbb{S}^{1}$, the circular package has functions for: descriptive statistics; KDE; pdf evaluation, simulation, and estimation for a range of classical and more recently proposed circular distributions; tests for uniformity, homogeneity, goodness-of-fit, and change points; one-way ANOVA. It also has functions for $\mathbb{S}^{1}-\mathbb{S}^{1}$ and $\mathbb{S}^{1}-\mathbb{R}$ regression and includes a variety of datasets. Many of circular's capabilities were illustrated in Pewsey et al. (2013). The latter's companion workspace, CircStatsInR, includes over 150 routines for techniques not implemented in circular.

Amongst its more specific capabilities, Directional implements techniques on $\mathbb{S}^{d}$ for: descriptive statistics; spherical data visualisation; constructing convenient rotation matrices and transformations; KDE; pdf computation, simulation, and ML estimation for various spherical distributions; $\mathbb{S}^{d}-\mathbb{S}^{d}$ correlation and regression, ANOVA, classification, and clustering.

\subsection{More specific $\mathrm{R}$ packages}

Here we provide an overview of more specific R packages and their functionality, following the order used to present themes in the previous sections. 
Various graphical representations for data on $\mathbb{S}^{1}$ are supported in bpDir (Buttarazzi, 2020), season (Barnett and Baker, 2020), and bReeze (Graul and Poppinga, 2018). Visualisation of data on $\mathbb{S}^{2}$ is facilitated by the outstanding rgl (Adler et al., 2020) and plot3D (Soetaert, 2019) packages.

Efficient modelling with vMF mixtures on $\mathbb{S}^{d}$ is implemented in movMF (Hornik and Grün, 2014). Several mixture models can be fitted using Bayesian methods to data on $\mathbb{S}^{1}$ and $\mathbb{T}^{2}$ with BAMBI (Chakraborty and Wong, 2019). Non-negative trigonometric sums can be fitted to data on $\mathbb{T}^{d}$ and $\mathbb{S}^{2}$ using CircnNTSR (Fernández-Durán and Gregorio-Domínguez, 2016).

Tests for uniformity and rotational symmetry on $\mathbb{S}^{d}$ are available in sphunif (García-Portugués and Verdebout, 2020) and rotasym (García-Portugués et al., 2020c), respectively.

Bayesian projected normal regression models for data on $\mathbb{S}^{1}$ are implemented in bpnreg (Cremers, 2020). Also for data on $\mathbb{S}^{1}$, nonparametric kernel methods for density and regression estimation are available in NPCirc (Oliveira et al., 2014). KDE and bandwidth selection on $\mathbb{S}^{d}$ are supported in DirStats (García-Portugués, 2020a). Nonparametric $\mathbb{S}^{d}-\mathbb{S}^{d}$ regression is implemented in nprotreg (Taylor et al., 2018). Smoothing splines on $\mathbb{S}^{2}$ are supported in mgcv (Wood, 2017).

Principal nested spheres and spherical $k$-means clustering can be performed with shapes (Dryden, 2019) and skmeans (Hornik et al., 2012), respectively.

Markov switching autoregressive models with vM innovations are implemented in NHMSAR (Monbet, 2020). Animal orientation data can be analysed using CircMLE (Fitak and Johnsen, 2017), FLightR (Rakhimberdiev et al., 2017), move (Kranstauber et al., 2020), and moveHMM (Michelot et al., 2016). Tools for toroidal diffusions are provided in sdetorus (García-Portugués, 2020b).

Bayesian methods for fitting spatial and spatio-temporal models to circular data are implemented in CircSpaceTime (Jona Lasinio et al., 2020). Spherical random fields can be analysed using RandomFields (Schlather et al., 2015). Routines for the management and analysis of cosmic microwave background data on $\mathbb{S}^{2}$ are available in rcosmo (Fryer et al., 2020).

Methods for analysing data on $\mathbb{S}^{1}$ under order restrictions are supported by isocir (Barragán et al., 2013). Outlier detection methods for $\mathbb{S}^{1}-\mathbb{S}^{1}$ regression are available in CircOutlier (Ghazanfarihesari and Sarmad, 2016). Depths on $\mathbb{S}^{1}$ and $\mathbb{S}^{2}$ can be computed using depth (Genest et al., 2019).

Intrinsic means and fundamental geodesic tools for $\mathbb{S}^{d}$ and other manifolds are available in RiemBase (You, 2020).

\section{Conclusions and future developments}

We hope that the previous sections provide both seasoned and neophyte researchers with a concise, comprehensive and useful overview of the widespread developments in directional statistics that have taken place over the last two decades. As often happens in research, most of those developments evolved in an uncoordinated way through the efforts of individuals and research groups working independently of one another. Given this background, predicting how the field might develop over the next 20 years is essentially impossible. That said, the further development of models with greater flexibility, techniques for high-dimensional and complex directional data involving combinations of different data types, as well as Bayesian, nonparametric, and resampling methods, would appear highly probable in the short term as such developments would be consistent with current trends. More generally, progress in all the areas covered in the previous sections is certainly possible and will no doubt evolve through responses to interesting new applications and the exigencies of the 
Riemannian supports of directional data, often incorporating appropriate adaptations of methodologies from other fields of statistics. The development of software to implement new techniques will continue to be crucial to the wider and proper application of directional statistics.

\section{Supplementary materials}

The BibTeX file DirectionalStats.bib includes entries for over 1700 references related to directional statistics. The file is available at https://github.com/egarpor/DirectionalStatsBib. We hope researchers in the field will find this resource useful.

\section{Acknowledgements}

We are most grateful to three anonymous referees and, in alphabetical order, to Davide Buttarazzi, Marco Di Marzio, Miguel Fernández, Stephan Huckemann, Peter Jupp, Shogo Kato, Christophe Ley, Kanti Mardia, and Louis-Paul Rivest, for their enthusiastic feedback on our original submission and helpful suggestions as to how it might be further improved. This work was supported by grants PGC2018-097284-B-100, IJCI-2017-32005, and MTM2016-76969-P from the Spanish Ministry of Economy and Competitiveness, and GR18016 from the Junta de Extremadura. All four grants were co-funded with FEDER funds from the European Union.

\section{References}

Abe, T. and Ley, C. (2017). A tractable, parsimonious and flexible model for cylindrical data, with applications. Econometrics and Statistics, 4:91-104. doi:10.1016/j.ecosta.2016.04.001.

Abe, T. and Pewsey, A. (2011). Sine-skewed circular distributions. Statistical Papers, 52(3):683-707. doi: $10.1007 / \mathrm{s} 00362-009-0277-\mathrm{x}$.

Abe, T. and Shimatani, I. K. (2018). Cylindrical distributions and their applications to biological data. In Ley, C. and Verdebout, T. (Eds.), Applied Directional Statistics, Chapman \& Hall/CRC Interdisciplinary Statistics Series, pp. 163-185. CRC Press, Boca Raton.

Abe, T., Shimizu, K., and Pewsey, A. (2010). Symmetric unimodal models for directional data motivated by inverse stereographic projection. Journal of the Japan Statistical Society, 40(1):4561. doi:10.14490/jjss.40.045.

Abraham, C., Molinari, N., and Servien, R. (2013). Unsupervised clustering of multivariate circular data. Statistics in Medicine, 32(8):1376-1382. doi:10.1002/sim. 5589.

Abraham, C., Servien, R., and Molinari, N. (2019). A clustering Bayesian approach for multivariate non-ordered circular data. Statistical Modelling, 19(6):595-616. doi:10.1177/ $1471082 X 18790420$.

Abuzaid, A. H., Hussin, A. G., and Mohamed, I. B. (2013). Detection of outliers in simple circular regression models using the mean circular error statistic. Journal of Statistical Computation and Simulation, 83(2):269-277. doi:10.1080/00949655.2011.602679.

Abuzaid, A. H., Mohamed, I. B., and Hussin, A. G. (2012). Boxplot for circular variables. Computational Statistics, 27(3):381-392. doi:10.1007/s00180-011-0261-5.

Abuzaid, A. H., Mohamed, I. B., and Hussin, A. G. (2014). Procedures for outlier detection in circular time series models. Environmental and Ecological Statistics, 21(4):793-809. doi:10. 1007/s10651-014-0281-8.

Adler, D., Murdoch, D., et al. (2020). rgl: 3D Visualization Using OpenGL. R package version 0.100.54. URL: https: //CRAN.R-project.org/package=rgl.

Agiomyrgiannakis, Y. and Stylianou, Y. (2009). Wrapped Gaussian mixture models for modeling and high-rate quantization of phase data of speech. IEEE Transactions on Audio Speech and Language Processing, 17(4):775-786. doi:10.1109/tasl.2008.2008229. 
Agostinelli, C. (2007). Robust estimation for circular data. Computational Statistics 83 Data Analysis, 51(12):5867-5875. doi:10.1016/j.csda.2006.11.002.

Agostinelli, C. and Lund, U. (2017). $R$ package circular: Circular Statistics. R package version 0.4-93. URL: https://CRAN.R-project.org/package=circular.

Agostinelli, C. and Romanazzi, M. (2013). Nonparametric analysis of directional data based on data depth. Environmental and Ecological Statistics, 20(2):253-270. doi:10.1007/ s10651-012-0218-z.

Ailliot, P., Bessac, J., Monbet, V., and Pène, F. (2015). Non-homogeneous hidden Markov-switching models for wind time series. Journal of Statistical Planning and Inference, 160:75-88. doi: $10.1016 / j . j$ spi.2014.12.005.

Aitchison, J. (1986). The Statistical Analysis of Compositional Data, volume 25 of Monographs on Statistics and Applied Probability. Chapman \& Hall, London. doi : 10.1007/978-94-009-4109-0.

Alonso-Pena, M., Ameijeiras-Alonso, J., and Crujeiras, R. M. (2019). Nonparametric tests for circular regression. arXiv:1910.07825.

Altis, A., Nguyen, P. H., Hegger, R., and Stock, G. (2007). Dihedral angle principal component analysis of molecular dynamics simulations. The Journal of Chemical Physics, 126(24):244111. doi:10.1063/1.2746330.

Amaral, G. J. A., Dryden, I. L., and Wood, A. T. A. (2007). Pivotal bootstrap methods for $k$ sample problems in directional statistics and shape analysis. Journal of the American Statistical Association, 102(478):695-707. doi:10.1198/016214506000001400.

Ameijeiras-Alonso, J., Benali, A., Crujeiras, R. M., Rodríguez-Casal, A., and Pereira, J. M. (2019a). Fire seasonality identification with multimodality tests. Annals of Applied Statistics, 13(4):21202139. doi:10.1214/19-AOAS1273.

Ameijeiras-Alonso, J., Crujeiras, R. M., and Rodríguez Casal, A. (2018). Directional statistics for wildfires. In Ley, C. and Verdebout, T. (Eds.), Applied Directional Statistics, Chapman \& Hall/CRC Interdisciplinary Statistics Series, pp. 187-210. CRC Press, Boca Raton.

Ameijeiras-Alonso, J., Lagona, F., Ranalli, M., and Crujeiras, R. M. (2019b). A circular nonhomogeneous hidden Markov field for the spatial segmentation of wildfire occurrences. Environmetrics, 30(2):e2501. doi:10.1002/env. 2501.

Ameijeiras-Alonso, J. and Ley, C. (2019). Sine-skewed toroidal distributions and their application in protein bioinformatics. arXiv:1910.13293.

Ameijeiras-Alonso, J., Ley, C., Pewsey, A., and Verdebout, T. (2020). On optimal tests for circular reflective symmetry about an unknown central direction. Statistical Papers, to appear. doi: $10.1007 / \mathrm{s} 00362-019-01150-7$.

Amiri, A., Thiam, B., and Verdebout, T. (2017). On the estimation of the density of a directional data stream. Scandinavian Journal of Statistics, 44(1):249-267. doi:10.1111/sjos.12252.

Anderson, C. M. (1993). Graphical methods for circular and cylindrical data. Technical report, Univeristy of Waterloo.

Anderson, T. W. (1963). Asymptotic theory for principal component analysis. Annals of Mathematical Statistics, 34(1):122-148. doi:10.1214/aoms/1177704248.

Arnold, B. C. and SenGupta, A. (2006). Recent advances in the analyses of directional data in ecological and environmental sciences. Environmental and Ecological Statistics, 13(3):253-256. doi:10.1007/s10651-006-0009-5.

Arnold, R. and Jupp, P. (2018). Orientations of symmetrical objects. In Ley, C. and Verdebout, T. (Eds.), Applied Directional Statistics, Chapman \& Hall/CRC Interdisciplinary Statistics Series, pp. 25-44. CRC Press, Boca Raton.

Artes, R. (2008). Hypothesis tests for covariance analysis models for circular data. Communications in Statistics - Theory and Methods, 37(8-10):1632-1640. doi:10.1080/03610920801893962.

Artes, R., Paula, G. A., and Ranvaud, R. (2000). Analysis of circular longitudinal data based on generalized estimating equations. Australian \& New Zealand Journal of Statistics, 42(3):347-358. doi:10.1111/1467-842x.00131. 
Artes, R. and Toloi, C. M. C. (2009). An autoregressive model for time series of circular data. Communications in Statistics - Theory and Methods, 39(1):186-194. doi:10.1080/ 03610920802650338.

Azzalini, A. (1985). A class of distributions which includes the normal ones. Scandinavian Journal of Statistics, 12(2):171-178.

Baayen, C. and Klugkist, I. (2014). Evaluating order-constrained hypotheses for circular data from a between-within subjects design. Psychological Methods, 19(3):398-408. doi:10.1037/a0037414.

Baayen, C., Klugkist, I., and Mechsner, F. (2012). A test of order-constrained hypotheses for circular data with applications to human movement science. Journal of Motor Behavior, 44(5):351-363. doi:10.1080/00222895.2012.709549.

Baba, Y. (1981). Statistics of angular data. Wrapped normal distribution model. Proceedings of the Institute of Statistical Mathematics, 28(1):41-54.

Bai, Z. D., Rao, C. R., and Zhao, L. C. (1988). Kernel estimators of density function of directional data. Journal of Multivariate Analysis, 27(1):24-39. doi:10.1016/0047-259X (88)90113-3.

Bakshaev, A. (2010). N-distance tests of uniformity on the hypersphere. Nonlinear Analysis: Modelling and Control, 15(1):15-8. doi:10.15388/na.2010.15.1.14361.

Baldi, P., Kerkyacharian, G., Marinucci, D., and Picard, D. (2009a). Adaptive density estimation for directional data using needlets. The Annals of Statistics, 37(6A):3362-3395. doi:10.1214/ $09-\operatorname{aos} 682$.

Baldi, P., Kerkyacharian, G., Marinucci, D., and Picard, D. (2009b). Asymptotics for spherical needlets. The Annals of Statistics, 37(3):1150-1171. doi:10.1214/08-A0S601.

Ball, F. G., Dryden, I. L., and Golalizadeh, M. (2008). Brownian motion and Ornstein-Uhlenbeck processes in planar shape space. Methodology and Computing in Applied Probability, 10(1):1-22. doi:10.1007/s11009-007-9042-6.

Banerjee, A., Dhillon, I., Ghosh, J., and Sra, S. (2003). Generative model-based clustering of directional data. In $K D D$ '03, pp. 19-28, New York. Association for Computing Machinery. doi:10.1145/956750.956757.

Banerjee, A., Dhillon, I. S., Ghosh, J., and Sra, S. (2005). Clustering on the unit hypersphere using von Mises-Fisher distributions. Journal of Machine Learning Research, 6(Sep):1345-1382.

Banerjee, A., Dhillon, I. S., Ghosh, J., and Sra, S. (2009). Text clustering with mixture of von Mises-Fisher distributions. In Srivastava, A. N. and Sahami, M. (Eds.), Text Mining, Chapman \& Hall/CRC Data Mining and Knowledge Discovery Series, pp. 151-184. CRC Press, New York. doi: 10.1201/9781420059458.

Bangert, M., Hennig, P., and Oelfke, U. (2010). Using an infinite von Mises-Fisher mixture model to cluster treatment beam directions in external radiation therapy. In $I C M L A$ '10, pp. 746-751, Washington D. C. IEEE Computer Society. doi:10.1109/icmla.2010.114.

Baragona, R. (2003). Further results on Lund's statistic for identifying cluster in a circular data set with application to time series. Communications in Statistics - Simulation and Computation, 32(3):943-952. doi:10.1081/sac-120017869.

Barnett, A. and Baker, P. (2020). season: Analysing Seasonal Data R Functions. R package version 0.3.12. URL: https://CRAN.R-project.org/package=season.

Barragán, S., Fernández, M. A., Rueda, C., and Peddada, S. (2013). isocir: an R package for constrained inference using isotonic regression for circular data, with an application to cell biology. Journal of Statistical Software, 54(4):1-17. doi:10.18637/jss.v054.i04.

Barragán, S., Rueda, C., and Fernández, M. A. (2017). Circular order aggregation and its application to cell-cycle genes expressions. IEEE/ACM Transactions on Computational Biology and Bioinformatics, 14(4):819-829.

Barragán, S., Rueda, C., Fernández, M. A., and Peddada, S. D. (2015). Determination of temporal order among the components of an oscillatory system. PLOS One, 10:e0124842. doi:10.1371/ journal pone. 0124842 .

Barros, C. M., Amaral, G. J. A., Nascimento, A. D. C., and Cysneiros, A. H. M. A. (2017). De- 
tecting influential observations in Watson data. Communications in Statistics - Simulation and Computation, 46(14):6882-6898. doi:10.1080/03610926.2016.1139130.

Batschelet, E. (1981). Circular Statistics in Biology. Mathematics in Biology. Academic Press, London.

Benjamin, J. B. M., Hussain, I., and Yang, M.-S. (2019). Possibilistic c-means clustering on directional data. In 2019 12th International Congress on Image and Signal Processing, BioMedical Engineering and Informatics (CISP-BMEI), pp. 1-6, New York. IEEE. doi:10.1109/ cisp-bmei48845.2019.8965703.

Beran, J. and Ghosh, S. (2020). Estimating the mean direction of strongly dependent circular time series. Journal of Time Series Analysis, 41:210-228. doi:10.1111/jtsa.12500.

Beran, R. (2016). Nonparametric estimation of trend in directional data. Stochastic Processes and their Applications, 126(12):3808-3827. doi:10.1016/j.spa.2016.04.018.

Beran, R. J. (1968). Testing for uniformity on a compact homogeneous space. Journal of Applied Probability, 5(1):177-195. doi:10.1017/s002190020003237x.

Beran, R. J. (1969). Asymptotic theory of a class of tests for uniformity of a circular distribution. Annals of Mathematical Statistics, 40(4):1196-1206. doi:10.1214/aoms/1177697496.

Beran, R. J. (1979). Exponential models for directional data. The Annals of Statistics, 7(6):11621178. doi:10.1214/aos/1176344838.

Berens, P. (2009). CircStat: a MATLAB toolbox for circular statistics. Journal of Statistical Software, 31(10):1-21. doi:10.18637/jss.v031.i10.

Bhattacharya, A. and Bhattacharya, R. (2012). Nonparametric Inference on Manifolds, volume 2 of Institute of Mathematical Statistics Monographs. Cambridge University Press, Cambridge. doi:10.1017/CB09781139094764.

Bhattacharya, A. and Dunson, D. (2012). Nonparametric Bayes classification and hypothesis testing on manifolds. Journal of Multivariate Analysis, 111:1-19. doi:10.1016/j.jmva.2012.02.020.

Bhattacharya, R. and Patrangenaru, V. (2003). Large sample theory of intrinsic and extrinsic sample means on manifolds. The Annals of Statistics, 31(1):1-29. doi:10.1214/aos/1046294456.

Bhattacharya, R. and Patrangenaru, V. (2005). Large sample theory of intrinsic and extrinsic sample means on manifolds-II. The Annals of Statistics, 33(3):1225-1259. doi:10.1214/ 009053605000000093.

Bhattacharya, R. and Patrangenaru, V. (2014). Statistics on manifolds and landmarks based image analysis: a nonparametric theory with applications. Journal of Statistical Planning and Inference, 145:1-22. doi:10.1016/j.jspi.2013.08.001.

Bhattacharya, S. and SenGupta, A. (2009a). Bayesian analysis of semiparametric linear-circular models. Journal of Agricultural, Biological, and Environmental Statistics, 14(1):33-65. doi: 10.1198/jabes. 2009.0003.

Bhattacharya, S. and SenGupta, A. (2009b). Bayesian inference for circular distributions with unknown normalising constants. Journal of Statistical Planning and Inference, 139(12):41794192. doi:10.1016/j.jspi.2009.06.008.

Bijral, A., Breitenbach, M., and Grudic, G. Z. (2007). Mixture of Watson distributions: a generative model for hyperspherical embeddings. In Meila, M. and Shen, X. (Eds.), Proceedings of the Eleventh International Conference on Artificial Intelligence and Statistics, Proceedings of Machine Learning Research, pp. 35-42, San Juan, Puerto Rico. PMLR.

Bingham, C. (1974). An antipodally symmetric distribution on the sphere. The Annals of Statistics, 2(6):1201-1225. doi:10.1214/aos/1176342874.

Boente, G. and Fraiman, R. (1991). Nonparametric regression for directional data. Trabajos de Matemática, 176:1-13.

Boente, G., Rodriguez, D., and González-Manteiga, W. (2014). Goodness-of-fit test for directional data. Scandinavian Journal of Statistics, 41(1):259-275. doi:10.1111/sjos.12020.

Bogdan, M., Bogdan, K., and Futschik, A. (2002). A data driven smooth test for circular uniformity. Annals of the Institute of Statistical Mathematics, 54(1):29-44. doi:10.1023/A:1016109603897. 
Boomsma, W., Mardia, K. V., Taylor, C. C., Ferkinghoff-Borg, J., Krogh, A., and Hamelryck, T. (2008). A generative, probabilistic model of local protein structure. Proceedings of the National Academy of Sciences of the United States of America, 105(26):8932-8937. doi:10.1073/pnas. 0801715105.

Boulerice, B. and Ducharme, G. R. (1997). Smooth tests of goodness-of-fit for directional and axial data. Journal of Multivariate Analysis, 60(1):154-174. doi:10.1006/jmva.1996.1650.

Breckling, J. (1989). The Analysis of Directional Time Series: Applications to Wind Speed and Direction, volume 61 of Lecture Notes in Statistics. Springer, London.

Bulla, J., Lagona, F., Maruotti, A., and Picone, M. (2012). A multivariate hidden Markov model for the identification of sea regimes from incomplete skewed and circular time series. Journal of Agricultural, Biological, and Environmental Statistics, 17(4):544-567. doi: 10.1007/s13253-012-0110-1.

Buttarazzi, D. (2020). bpDir: Boxplots for Directional Data. R package version 0.1.1. URL: https : //CRAN.R-project . org/package=bpDir.

Buttarazzi, D., Pandolfo, G., and Porzio, G. C. (2018). A boxplot for circular data. Biometrics, 74(4):1492-1501. doi:10.1111/biom. 12889.

Byrne, R. W., Noser, R., Bates, L. A., and Jupp, P. E. (2009). How did they get here from there? detecting changes of direction in terrestrial ranging. Animal Behaviour, 77(3):619-631. doi:10.1016/j.anbehav.2008.11.014.

Byrne, S. and Girolami, M. (2013). Geodesic Monte Carlo on embedded manifolds. Scandinavian Journal of Statistics, 40(4):825-845. doi:10.1111/sjos.12036.

Cabella, P. and Marinucci, D. (2009). Statistical challenges in the analysis of cosmic microwave background radiation. The Annals of Applied Statistics, 3(1):61-95. doi:10.1214/08-aoas190.

Cai, T., Fan, J., and Jiang, T. (2013). Distributions of angles in random packing on spheres. Journal of Machine Learning Research, 14(21):1837-1864.

Cai, T. and Jiang, T. (2012). Phase transition in limiting distributions of coherence of highdimensional random matrices. Journal of Multivariate Analysis, 107:24-39. doi:10.1016/j . jmva.2011.11.008.

Calderara, S., Prati, A., and Cucchiara, R. (2011). Mixtures of von Mises distributions for people trajectory shape analysis. IEEE Transactions on Circuits and Systems for Video Technology, 21(4):457-471. doi:10.1109/tcsvt.2011.2125550.

Carnicero, J. A., Wiper, M. P., and Ausín, M. C. (2018). Density estimation of circular data with Bernstein polynomials. Hacettepe Journal of Mathematics and Statistics, 47(2):273-286. doi : 10.15672/hjms. 2014437525.

Carta, J. A., Ramirez, P., and Bueno, C. (2008). A joint probability density function of wind speed and direction for wind energy analysis. Energy Conversion and Management, 49(6):1309-1320. doi: $10.1016 / j$.enconman.2008.01.010.

Cetingul, H. E. and Vidal, R. (2009). Intrinsic mean shift for clustering on Stiefel and Grassmann manifolds. In 2009 IEEE Conference on Computer Vision and Pattern Recognition, pp. 18961902, New York. IEEE. doi:10.1109/cvpr.2009.5206806.

Chakraborty, S. and Wong, S. W. K. (2019). BAMBI: Bivariate Angular Mixture Models. R package version 2.3.0. URL: https://CRAN.R-project.org/package=BAMBI.

Chang, T. (1986). Spherical regression. The Annals of Statistics, 14(3):907-924. doi:10.1002/ 0471667196. ess0734.

Chang-Chien, S.-J., Yang, M.-S., and Hung, W.-L. (2010). Mean shift-based clustering for directional data. In Third International Workshop on Advanced Computational Intelligence, pp. 367-372. doi:10.1109/IWACI .2010.5585203.

Chaubey, Y. P. (2018). Smooth kernel estimation of a circular density function: a connection to orthogonal polynomials on the unit circle. Journal of Probability and Statistics, 2018:1-4. doi:10.1155/2018/5372803.

Chaudhuri, P. and Marron, J. S. (1999). SiZer for exploration of structures in curves. Journal of 
the American Statistical Association, 94(447):807-823. doi:10.1080/01621459.1999.10474186. Cheng, D., Cammarota, V., Fantaye, Y., Marinucci, D., and Schwartzman, A. (2020). Multiple testing of local maxima for detection of peaks on the (celestial) sphere. Bernoulli, 26(1):31-60. doi:10.3150/18-bej1068.

Cheng, M.-Y. and Wu, H.-T. (2013). Local linear regression on manifolds and its geometric interpretation. Journal of the American Statistical Association, 108(504):1421-1434. doi: 10.1080/01621459.2013.827984.

Chikuse, Y. (2012). Statistics on Special Manifolds, volume 174 of Lecture Notes in Statistics. Springer, Heidelberg. doi : 10.1007/978-0-387-21540-2.

Chirikjian, G. S. and Kyatkin, A. (2001). Engineering Applications of Noncommutative Harmonic Analysis. CRC Press, Boca Raton. doi:10.1115/1.1421108.

Chiuso, A. and Picci, G. (1998). Visual tracking of points as estimation on the unit sphere. In Kriegman, D. J., Hager, G. D., and Morse, A. S. (Eds.), The Confluence of Vision and Control, volume 237 of Lecture Notes in Control and Information Sciences, pp. 90-105, London. Springer. doi: 10.1007/BFb0109665.

Codling, E. A., Plank, M. J., and Benhamou, S. (2008). Random walk models in biology. Journal of the Royal Society Interface, 5(25):813-834. doi:10.1098/rsif.2008.0014.

Comte, F. and Taupin, M. L. (2003). Adaptive density deconvolution for circular data. Technical Report MAP5 2003-10, Université Paris Descartes.

Cornea, E., Zhu, H., Kim, P., and Ibrahim, J. G. (2017). Regression models on Riemannian symmetric spaces. Journal of the Royal Statistical Society, Series B (Statistical Methodology), 79(2):463482. doi:10.1111/rssb.12169.

Costa, M., Koivunen, V., and Poor, H. V. (2014). Estimating directional statistics using wavefield modeling and mixtures of von-Mises distributions. IEEE Signal Processing Letters, 21(12):14961500. doi:10.1109/1sp.2014.2341651.

Cremers, J. (2020). bpnreg: Bayesian Projected Normal Regression Models for Circular Data. R package version 1.0.3. URL: https://CRAN. R-project .org/package=bpnreg.

Cremers, J., Mulder, K. T., and Klugkist, I. (2018). Circular interpretation of regression coefficients. British Journal of Mathematical and Statistical Psychology, 71(1):75-95. doi:10.1111/bmsp. 12108.

Cremers, J., Pennings, H. J. M., and Ley, C. (2020). Regression models for cylindrical data in psychology. Multivariate Behavioral Research, to appear. doi:10.1080/00273171.2019.1693332.

Cuesta-Albertos, J. A., Cuevas, A., and Fraiman, R. (2009). On projection-based tests for directional and compositional data. Statistics and Computing, 19(4):367-380. doi:10.1007/ s11222-008-9098-3.

Curry, C., Marsland, S., and McLachlan, R. I. (2019). Principal symmetric space analysis. Journal of Computational Dynamics, 6(2):251-276. doi:10.3934/jcd.2019013.

Cutting, C., Paindaveine, D., and Verdebout, T. (2017a). Testing uniformity on high-dimensional spheres against monotone rotationally symmetric alternatives. The Annals of Statistics, 45(3):1024-1058. doi:10.1214/16-aos1473.

Cutting, C., Paindaveine, D., and Verdebout, T. (2017b). Tests of concentration for low-dimensional and high-dimensional directional data. In Ahmed, S. E. (Ed.), Big and Complex Data Analysis, Contributions to Statistics. Springer, New York. doi:10.1007/978-3-319-41573-4_11.

Cutting, C., Paindaveine, D., and Verdebout, T. (2020). On the power of axial tests of uniformity on spheres. Electronic Journal of Statistics, 14(1):2123-2154. doi:10.1214/20-EJS1716.

Dai, F. and Xu, Y. (2013). Approximation Theory and Harmonic Analysis on Spheres and Balls. Springer Monographs in Mathematics. Springer, New York. doi:10.1007/978-1-4614-6660-4.

Dai, X. and Müller, H.-G. (2018). Principal component analysis for functional data on Riemannian manifolds and spheres. The Annals of Statistics, 46(6B):3334-3361. doi:10.1214/17-aos1660.

Damien, P. and Walker, S. (1999). A full Bayesian analysis of circular data using the von Mises distribution. The Canadian Journal of Statistics, 27(2):291-298. doi:10.2307/3315639. 
Damon, J. and Marron, J. S. (2014). Backwards principal component analysis and principal nested relations. Journal of Mathematical Imaging and Vision, 50(1):107-114. doi:10.1007/ s10851-013-0463-2.

D'Elia, A. (2001). A statistical model for orientation mechanism. Statistical Methods and Applications, 10(1-3):157-174. doi:10.1007/BF02511646.

Demni, H., Messaoud, A., and Porzio, G. C. (2019). The cosine depth distribution classifier for directional data. In Bauer, N., Ickstadt, K., Lübke, K., Szepannek, G., Trautmann, H., and Vichi, M. (Eds.), Applications in Statistical Computing, Studies in Classification, Data Analysis, and Knowledge Organization, pp. 49-60. Springer, Cham. doi:10.1007/978-3-030-25147-5_4.

Deschepper, E., Thas, O., and Ottoy, J.-P. (2008). Tests and diagnostic plots for detecting lack-offit for circular-linear regression models. Biometrics, 64(3):912-920. doi:10.1111/j.1541-0420. 2007.00950.x.

Dette, H., Konstantinou, M., Schorning, K., and Gösmann, J. (2019). Optimal designs for regression with spherical data. Electronic Journal of Statistics, 13(1):361-390. doi:10.1214/18-ejs1524.

Dette, H. and Melas, V. B. (2003). Optimal designs for estimating individual coefficients in Fourier regression models. The Annals of Statistics, 31(5):1669-1692. doi:10.1214/aos/1065705122.

Dette, H., Melas, V. B., and Pepelyshev, A. (2005). Optimal designs for three-dimensional shape analysis with spherical harmonic descriptors. The Annals of Statistics, 33(6):2758-2788. doi: 10.1214/009053605000000552.

Dette, H. and Wiens, D. P. (2009). Robust designs for 3D shape analysis with spherical harmonic descriptors. Statistica Sinica, 19(1):83-102.

Dhillon, I. and Sra, S. (2003). Modeling data using directional distributions. Technical Report TR-03-06, Department of Computer Sciences, University of Texas at Austin.

Dhillon, I. S. and Modha, D. S. (2001). Concept decompositions for large sparse text data using clustering. Machine Learning, 42(1):143-175. doi:10.1023/A:1007612920971.

Di Marzio, M., Fensore, S., Panzera, A., and Taylor, C. C. (2016a). A note on nonparametric estimation of circular conditional densities. Journal of Statistical Computation and Simulation, 86(13):2573-2582. doi:10.1080/00949655.2016.1146279.

Di Marzio, M., Fensore, S., Panzera, A., and Taylor, C. C. (2016b). Practical performance of local likelihood for circular density estimation. Journal of Statistical Computation and Simulation, 86(13):2560-2572. doi:10.1080/00949655.2016.1149588.

Di Marzio, M., Fensore, S., Panzera, A., and Taylor, C. C. (2017). Nonparametric estimating equations for circular probability density functions and their derivatives. Electronic Journal of Statistics, 11(2):4323-4346. doi:10.1214/17-EJS1318.

Di Marzio, M., Fensore, S., Panzera, A., and Taylor, C. C. (2018a). Circular local likelihood. TEST, 27(4):921-945. doi:10.1007/s11749-017-0576-9.

Di Marzio, M., Fensore, S., Panzera, A., and Taylor, C. C. (2018b). Nonparametric classification for circular data. In Ley, C. and Verdebout, T. (Eds.), Applied Directional Statistics, Chapman \& Hall/CRC Interdisciplinary Statistics Series, pp. 241-257. CRC Press, Boca Raton.

Di Marzio, M., Fensore, S., Panzera, A., and Taylor, C. C. (2019a). Kernel density classification for spherical data. Statistics \& Probability Letters, 144:23-29. doi:10.1016/j.spl.2018.07.018.

Di Marzio, M., Fensore, S., Panzera, A., and Taylor, C. C. (2019b). Local binary regression with spherical predictors. Statistics \& Probability Letters, 144:30-36. doi:10.1016/j.spl.2018.07. 019.

Di Marzio, M., Panzera, A., and Taylor, C. C. (2009). Local polynomial regression for circular predictors. Statistics \& Probability Letters, 79(19):2066-2075. doi:10.1016/j .spl.2009.06.014.

Di Marzio, M., Panzera, A., and Taylor, C. C. (2011). Kernel density estimation on the torus. Journal of Statistical Planning and Inference, 141(6):2156-2173. doi:10.1016/j.jspi.2011.01.002.

Di Marzio, M., Panzera, A., and Taylor, C. C. (2012a). Non-parametric smoothing and prediction for nonlinear circular time series. Journal of Time Series Analysis, 33(4):620-630. doi:10.1111/ j.1467-9892.2012.00794.x. 
Di Marzio, M., Panzera, A., and Taylor, C. C. (2012b). Smooth estimation of circular cumulative distribution functions and quantiles. Journal of Nonparametric Statistics, 24(4):935-949. doi: $10.1080 / 10485252.2012 .721517$.

Di Marzio, M., Panzera, A., and Taylor, C. C. (2013). Non-parametric regression for circular responses. Scandinavian Journal of Statistics, 40(2):238-255. doi:10.1111/j .1467-9469.2012. 00809.x.

Di Marzio, M., Panzera, A., and Taylor, C. C. (2014). Nonparametric regression for spherical data. Journal of the American Statistical Association, 109(506):748-763. doi:10.1080/01621459. 2013.866567.

Di Marzio, M., Panzera, A., and Taylor, C. C. (2016c). Nonparametric circular quantile regression. Journal of Statistical Planning and Inference, 170:1-14. doi:10.1016/j.jspi.2015.08.004.

Di Marzio, M., Panzera, A., and Taylor, C. C. (2019c). Nonparametric rotations for sphere-sphere regression. Journal of the American Statistical Association, 114(525):466-476. doi:10.1080/ 01621459.2017.1421542.

Dokmanic, I. and Petrinovic, D. (2010). Convolution on the $n$-sphere with application to pdf modeling. IEEE Transactions on Signal Processing, 58(3):1157-1170. doi:10.1109/TSP. 2009. 2033329.

Dortet-Bernadet, J.-L. and Wicker, N. (2008). Model-based clustering on the unit sphere with an illustration using gene expression profiles. Biostatistics, 9(1):66-80. doi:10.1093/biostatistics/ kxm012.

Downs, T. D. (2003). Spherical regression. Biometrika, 90(3):655-668. doi:10.1093/biomet/90. 3.655.

Downs, T. D. and Mardia, K. V. (2002). Circular regression. Biometrika, 89(3):683-697. doi: 10.1093/biomet/89.3.683.

Dryden, I. L. (2019). shapes: Statistical Shape Analysis. R package version 1.2.5. URL: https: //CRAN.R-project . org/package=shapes.

Dryden, I. L. and Mardia, K. V. (2016). Statistical Shape Analysis with Applications in $R$. Wiley Series in Probability and Statistics. Wiley, Chichester, second edition. doi:10.1002/ 9781119072492.

Ducharme, G. R., Vincent, C., and Aliaume, C. (2012). A statistical test to detect vortices in the current fields of bodies of water. Environmental and Ecological Statistics, 19(3):345-367. doi:10.1007/s10651-012-0190-7.

Ebner, B., Henze, N., and Yukich, J. E. (2018). Multivariate goodness-of-fit on flat and curved spaces via nearest neighbor distances. Journal of Multivariate Analysis, 165:231-242. doi: $10.1016 / j$.jmva.2017.12.009.

Efromovich, S. (1997). Density estimation for the case of supersmooth measurement error. Journal of the American Statistical Association, 92(438):526-535. doi:10.2307/2965701.

Ehler, M. and Galanis, J. (2011). Frame theory in directional statistics. Statistics \& Probability Letters, 81(8):1046-1051. doi:10.1016/j.spl.2011.02.027.

Eisen, M. B., Spellman, P. T., Brown, P. O., and Botstein, D. (1998). Cluster analysis and display of genome-wide expression patterns. Proceedings of the National Academy of Sciences of the United States of America, 95(25):14863-14868. doi:10.1073/pnas.95.25.14863.

Elad, A., Keller, Y., and Kimmel, R. (2005). Texture mapping via spherical multi-dimensional scaling. In Kimmel, R., Sochen, N. A., and Weickert, J. (Eds.), Scale Space and PDE Methods in Computer Vision, volume 3459 of Lecture Notes in Computer Science, pp. 443-455, Berlin. Springer. doi:10.1007/11408031_38.

Eltzner, B., Huckemann, S., and Mardia, K. V. (2018). Torus principal component analysis with applications to RNA structure. The Annals of Applied Statistics, 12(2):1332-1359. doi:10.1214/ 17-AOAS1115.

Eltzner, B. and Huckemann, S. F. (2019). A smeary central limit theorem for manifolds with application to high-dimensional spheres. The Annals of Statistics, 47(6):3360-3381. doi:10. 
1214/18-AOS1781.

Eltzner, B., Jung, S., and Huckemann, S. (2015). Dimension reduction on polyspheres with application to skeletal representations. In Nielsen, F. and Barbaresco, F. (Eds.), Geometric Science of Information, volume 9389 of Lecture Notes in Computer Science, pp. 22-29, Cham. Springer. doi:10.1007/978-3-319-25040-3_3.

Erdem, E. and Shi, J. (2011). Comparison of bivariate distribution construction approaches for analysing wind speed and direction data. Wind Energy, 14(1):27-41. doi:10.1002/we.400.

Esteves, C., Allen-Blanchette, C., Makadia, A., and Daniilidis, K. (2020). Learning SO(3) equivariant representations with spherical CNNs. International Journal of Computer Vision, 128:588-600. doi:10.1007/s11263-019-01220-1.

Ĕgecioğlu, O. and Srinivasan, A. (2000). Efficient nonparametric density estimation on the sphere with applications in fluid mechanics. SIAM Journal on Scientific Computing, 22(1):152-176. doi:10.1137/S1064827595290462.

Fallaize, C. J. and Kypraios, T. (2016). Exact Bayesian inference for the Bingham distribution. Statistics and Computing, 26(1-2):349-360. doi:10.1007/s11222-014-9508-7.

Faÿ, G., Delabrouille, J., Kerkyacharian, G., and Picard, D. (2013). Testing the isotropy of high energy cosmic rays using spherical needlets. The Annals of Applied Statistics, 7(2):1040-1073. doi:10.1214/12-aoas619.

Fejér, L. (1916). Über trigonometrische Polynome. Journal für die reine und angewandte Mathematik, 146:53-82. doi:10.1515/crll.1916.146.53.

Feltz, C. J. and Goldin, G. A. (2001). Partition-based goodness-of-fit tests on the line and the circle. Australian \& New Zealand Journal of Statistics, 43(2):207-220. doi:10.1111/1467-842X .00166.

Fernandes, K. and Cardoso, J. S. (2016). Discriminative directional classifiers. Neurocomputing, 207:141-149. doi:10.1016/j.neucom.2016.03.076.

Fernández, M. A., Rueda, C., and Peddada, S. D. (2012). Identification of a core set of signature cell cycle genes whose relative order of time to peak expression is conserved across species. Nucleic Acids Research, 40(7):2823-2832. doi:10.1093/nar/gkr1077.

Fernández-Durán, J. J. (2004). Circular distributions based on nonnegative trigonometric sums. Biometrics, 60(2):499-503. doi:10.1111/j.0006-341x.2004.00195.x.

Fernández-Durán, J. J. and Gregorio-Domínguez, M. M. (2014a). Distributions for spherical data based on nonnegative trigonometric sums. Statistical Papers, 55(4):983-1000. doi:10.1007/ s00362-013-0547-5.

Fernández-Durán, J. J. and Gregorio-Domínguez, M. M. (2014b). Modeling angles in proteins and circular genomes using multivariate angular distributions based on multiple nonnegative trigonometric sums. Statistical Applications in Genetics and Molecular Biology, 13(1):1-18. doi: 10.1515/sagmb-2012-0012.

Fernández-Durán, J. J. and Gregorio-Domínguez, M. M. (2016). CircNNTSR: an R package for the statistical analysis of circular, multivariate circular, and spherical data using nonnegative trigonometric sums. Journal of Statistical Software, 70(6):1-19. doi:10.18637/jss.v070.i06.

Ferreira, J. T. A. S., Juárez, M. A., and Steel, M. F. J. (2008). Directional log-spline distributions. Bayesian Analysis, 3(2):297-316. doi:10.1214/08-ba311.

Figueiredo, A. (2007). Comparison of tests of uniformity defined on the hypersphere. Statistics 86 Probability Letters, 77(3):329-334. doi:10.1016/j.spl.2006.07.012.

Figueiredo, A. (2009). Discriminant analysis for the von Mises-Fisher distribution. Communications in Statistics - Simulation and Computation, 38(9):1991-2003. doi:10.1080/03610910903200281.

Figueiredo, A. (2017). Bootstrap and permutation tests in ANOVA for directional data. Computational Statistics, 32(4):1213-1240. doi:10.1007/s00180-017-0739-x.

Figueiredo, A. and Gomes, P. (2003). Power of tests of uniformity defined on the hypersphere. Communications in Statistics - Simulation and Computation, 32(1):87-94. doi: 10.1081/sac-120013113.

Figueiredo, A. and Gomes, P. (2005). Discordancy test for the bipolar Watson distribution defined 
on the hypersphere. Communications in Statistics - Simulation and Computation, 34(1):145-153. doi: $10.1081 / \mathrm{sac}-200047092$.

Figueiredo, A. and Gomes, P. (2006). Discriminant analysis based on the Watson distribution defined on the hypersphere. Statistics, 40(5):435-445. doi:10.1080/02331880600766662.

Fisher, N. I. (1993). Statistical Analysis of Circular Data. Cambridge University Press, Cambridge. doi: $10.1017 /$ cbo9780511564345.

Fisher, N. I. and Lee, A. J. (1992). Regression models for an angular response. Biometrics, 48(3):665677. doi:10.2307/2532334.

Fisher, N. I. and Lee, A. J. (1994). Time series analysis of circular data. Journal of the Royal Statistical Society, Series B (Methodological), 56(2):327-339. doi:10.1111/j.2517-6161.1994. tb01981.x.

Fisher, N. I., Lewis, T., and Embleton, B. J. (1987). Statistical Analysis of Spherical Data. Cambridge University Press, Cambridge. doi:10.1017/cbo9780511623059.

Fisher, N. I. and Marron, J. S. (2001). Mode testing via the excess mass estimate. Biometrika, 88(2):499-517. doi:10.1093/biomet/88.2.499.

Fitak, R. R. and Johnsen, S. (2017). Bringing the analysis of animal orientation data full circle: model-based approaches with maximum likelihood. Journal of Experimental Biology, 220(21):3878-3882. doi:10.1242/jeb. 167056.

Fletcher, P. T., Lu, C., Pizer, S. M., and Joshi, S. (2004). Principal geodesic analysis for the study of nonlinear statistics of shape. IEEE Transactions on Medical Imaging, 23(8):995-1005. doi:10.1109/tmi.2004.831793.

Franke, J., Redenbach, C., and Zhang, N. (2016). On a mixture model for directional data on the sphere. Scandinavian Journal of Statistics, 43(1):139-155. doi:10.1111/sjos.12169.

Fryer, D., Olenko, A., Li, M., and Wang, Y. (2020). rcosmo: Cosmic Microwave Background Data Analysis. R package version 1.1.2. URL: https://CRAN.R-project.org/package=rcosmo.

$\mathrm{Fu}$, Y., Chen, J., and Li, P. (2008). Modified likelihood ratio test for homogeneity in a mixture of von Mises distributions. Journal of Statistical Planning and Inference, 138(3):667-681. doi: $10.1016 / j . j$ spi.2007.01.003.

Gao, F. Q. and Li, L. N. (2010). Large deviations and moderate deviations for kernel density estimators of directional data. Acta Mathematica Sinica, English Series, 26(5):937-950. doi: $10.1007 / \mathrm{s} 10114-010-7205-9$.

García-Portugués, E. (2013). Exact risk improvement of bandwidth selectors for kernel density estimation with directional data. Electronic Journal of Statistics, 7:1655-1685. doi:10.1214/ 13-ejs821.

García-Portugués, E. (2020a). DirStats: Nonparametric Methods for Directional Data. R package version 0.1.6. URL: https://CRAN.R-project.org/package=DirStats.

García-Portugués, E. (2020b). sdetorus: Statistical Tools for Toroidal Diffusions. R package version 0.1.7. URL: https://CRAN.R-project.org/package=sdetorus.

García-Portugués, E., Barros, A. M. G., Crujeiras, R. M., González-Manteiga, W., and Pereira, J. (2014). A test for directional-linear independence, with applications to wildfire orientation and size. Stochastic Environmental Research and Risk Assessment, 28(5):1261-1275. doi:10.1007/ s00477-013-0819-6.

García-Portugués, E., Crujeiras, R. M., and González-Manteiga, W. (2013a). Exploring wind direction and $\mathrm{SO}_{2}$ concentration by circular-linear density estimation. Stochastic Environmental Research and Risk Assessment, 27(5):1055-1067. doi:10.1007/s00477-012-0642-5.

García-Portugués, E., Crujeiras, R. M., and González-Manteiga, W. (2013b). Kernel density estimation for directional-linear data. Journal of Multivariate Analysis, 121:152-175. doi: $10.1016 / j$.jmva.2013.06.009.

García-Portugués, E., Crujeiras, R. M., and González-Manteiga, W. (2015). Central limit theorems for directional and linear random variables with applications. Statistica Sinica, 25(3):1207-1229. doi:10.5705/ss.2014.153. 
García-Portugués, E., Navarro-Esteban, P., and Cuesta-Albertos, J. A. (2020a). On a projectionbased class of uniformity tests on the hypersphere. arXiv:2008.0989\%.

García-Portugués, E., Paindaveine, D., and Verdebout, T. (2020b). On optimal tests for rotational symmetry against new classes of hyperspherical distributions. Journal of the American Statistical Association, to appear. doi:10.1080/01621459.2019.1665527.

García-Portugués, E., Paindaveine, D., and Verdebout, T. (2020c). rotasym: Tests for Rotational Symmetry on the Hypersphere. R package version 1.0.9. URL: https://CRAN.R-project.org/ package $=$ rotasym.

García-Portugués, E., Sørensen, M., Mardia, K. V., and Hamelryck, T. (2019). Langevin diffusions on the torus: estimation and applications. Statistics and Computing, 29(1):1-22. doi:10.1007/ s11222-017-9790-2.

García-Portugués, E., Van Keilegom, I., Crujeiras, R. M., and González-Manteiga, W. (2016). Testing parametric models in linear-directional regression. Scandinavian Journal of Statistics, 43(4):1178-1191. doi:10.1111/sjos.12236.

García-Portugués, E. and Verdebout, T. (2018). A review of uniformity tests on the hypersphere. arXiv:1804.00286.

García-Portugués, E. and Verdebout, T. (2020). sphunif: Uniformity Tests on the Circle, Sphere, and Hypersphere. URL: https://github.com/egarpor/sphunif.

Gatto, R. (2000). Multivariate saddlepoint test for the wrapped normal model. Journal of Statistical Computation and Simulation, 65(1-4):271-285. doi:10.1080/00949650008812002.

Gatto, R. (2006). A bootstrap test for circular data. Communications in Statistics - Theory and Methods, 35(1-3):281-292. doi:10.1080/03610920500440057.

Gatto, R. (2008). Some computational aspects of the generalized von Mises distribution. Statistics and Computing, 18(3):321-331. doi:10.1007/s11222-008-9060-4.

Gatto, R. (2009). Information theoretic results for circular distributions. Statistics, 43(4):409-421. doi: 10.1080/09603100802395947.

Gatto, R. (2017). Multivariate saddlepoint tests on the mean direction of the von Mises-Fisher distribution. Metrika, 80(6-8):733-747. doi:10.1007/s00184-017-0625-0.

Genest, M., Masse, J.-C., and Plante, J.-F. (2019). depth: Nonparametric Depth Functions for Multivariate Analysis. R package version 2.1-1.1. URL: https://CRAN.R-project.org/package= depth.

Genton, M. G. and Hall, P. (2007). Statistical inference for evolving periodic functions. Journal of the Royal Statistical Society, Series B (Statistical Methodology), 69(4):643-657. doi:10.1111/j . 1467-9868.2007.00604.x.

George, B. J. and Ghosh, K. (2006). A semiparametric Bayesian model for circular-linear regression. Communications in Statistics - Simulation and Computation, 35(4):911-923. doi: 10.1080/03610910600880302.

Ghazanfarihesari, A. and Sarmad, M. (2016). CircOutlier: Detection of Outliers in CircularCircular Regression. R package version 3.2.3. URL: https://CRAN.R-project.org/package= CircOutlier.

Ghosh, K., Jammalamadaka, S. R., and Vasudaven, M. (1999). Change-point problems for the von Mises distribution. Journal of Applied Statistics, 26(4):423-434. doi:10.1080/02664769922313.

Ghosh, M., Zhong, X., SenGupta, A., and Zhang, R. (2019). Non-subjective priors for wrapped Cauchy distributions. Statistics \& Probability Letters, 153:90-97. doi:10.1016/j .spl.2019.05. 016.

Gill, J. and Hangartner, D. (2010). Circular data in political science and how to handle it. Political Analysis, 18(3):316-336. doi:10.1093/pan/mpq009.

Giné, E. (1975). Invariant tests for uniformity on compact Riemannian manifolds based on Sobolev norms. The Annals of Statistics, 3(6):1243-1266. doi:10.1214/aos/1176343283.

Giummolè, F., Mameli, V., Ruli, E., and Ventura, L. (2019). Objective Bayesian inference with proper scoring rules. TEST, 28(3):728-755. doi:10.1007/s11749-018-0597-z. 
Gneiting, T. (2013). Strictly and non-strictly positive definite functions on spheres. Bernoulli, 19(4):1327-1349. doi:10.3150/12-bejsp06.

Godtliebsen, F., Marron, J. S., and Chaudhuri, P. (2002). Significance in scale space for bivariate density estimation. Journal of Computational and Graphical Statistics, 11(1):1-21. doi:10.1198/ 106186002317375596.

Golden, M., García-Portugués, E., Sørensen, M., Mardia, K. V., Hamelryck, T., and Hein, J. (2017). A generative angular model of protein structure evolution. Molecular Biology and Evolution, 34(8):2085-2100. doi:10.1093/molbev/msx137.

Gopal, S. and Yang, Y. (2014). Von Mises-Fisher clustering models. In Xing, E. P. and Jebara, T. (Eds.), Proceedings of the 31st International Conference Machine Learning, volume 32 of Proceedings of Machine Learning Research, pp. 154-162, Bejing. PMLR.

Graul, C. and Poppinga, C. (2018). bReeze: Functions for Wind Resource Assessment. R package version 0.4-3. URL: https://CRAN.R-project.org/package=bReeze.

Gu, X., Wang, Y., Chan, T. F., Thompson, P. M., and Yau, S.-T. (2004). Genus zero surface conformal mapping and its application to brain surface mapping. IEEE Transactions on Medical Imaging, 23(8):949-958.

Guella, J. C., Menegatto, V. A., and Porcu, E. (2018). Strictly positive definite multivariate covariance functions on spheres. Journal of Multivariate Analysis, 166:150-159. doi: $10.1016 / j$. jmva.2018.03.001.

Hall, P., Reimann, J., and Rice, J. (2000). Nonparametric estimation of a periodic function. Biometrika, 87(3):545-557. doi:10.1093/biomet/87.3.545.

Hall, P., Watson, G. S., and Cabrera, J. (1987). Kernel density estimation with spherical data. Biometrika, 74(4):751-762. doi:10.1093/biomet/74.4.751.

Hall, P. and Yin, J. (2003). Nonparametric methods for deconvolving multiperiodic functions. Journal of the Royal Statistical Society, Series B (Statistical Methodology), 65(4):869-886. doi: $10.1046 / j .1369-7412.2003 .00420 . x$.

Hamsici, O. C. and Martinez, A. M. (2007). Spherical-homoscedastic distributions: the equivalency of spherical and normal distributions in classification. Journal of Machine Learning Research, 8(Jul):1583-1623.

Hara, K., Nishino, K., and Ikeuchi, K. (2008). Mixture of spherical distributions for single-view relighting. IEEE Transactions on Pattern Analysis and Machine Intelligence, 30(1):25-35. doi: 10.1109/tpami.2007.1164.

Hartman, P. and Watson, G. S. (1974). "normal" distribution functions on spheres and the modified Bessel functions. The Annals of Probability, 2(4):593-607. doi:10.1214/aop/1176996606.

Hasnat, M. A., Alata, O., and Trémeau, A. (2014). Unsupervised clustering of depth images using Watson mixture model. In 2014 22nd International Conference on Pattern Recognition, pp. 214-219, New York. IEEE. doi:10.1109/icpr.2014.46.

Hassanzadeh, F. and Kalaylioglu, Z. (2018). A new multimodal and asymmetric bivariate circular distribution. Environmental and Ecological Statistics, 25(3):363-385. doi:10.1007/ s10651-018-0409-3.

Hawkins, D. M. and Lombard, F. (2015). Segmentation of circular data. Journal of Applied Statistics, 42(1):88-97. doi:10.1080/02664763.2014.934665.

Hawkins, D. M. and Lombard, F. (2017). Cusum control for data following the von Mises distribution. Journal of Applied Statistics, 44(8):1319-1332.

Healy, D. M. J., Hendriks, H., and Kim, P. T. (1998). Spherical deconvolution. Journal of Multivariate Analysis, 67(1):1-22. doi:10.1006/jmva.1998.1757.

Henry, G. and Rodriguez, D. (2009). Kernel density estimation on Riemannian manifolds: asymptotic results. Journal of Mathematical Imaging and Vision, 34(3):235-239. doi:10.1007/ s10851-009-0145-2.

Hernandez-Stumpfhauser, D., Breidt, F. J., and Opsomer, J. D. (2016). Hierarchical Bayesian small area estimation for circular data. The Canadian Journal of Statistics, 44(4):416-430. doi: 
$10.1002 /$ cjs. 11303.

Hernandez-Stumpfhauser, D., Breidt, F. J., and van der Woerd, M. J. (2017). The general projected normal distribution of arbitrary dimension: modeling and Bayesian inference. Bayesian Analysis, 12(1):113-133. doi:10.1214/15-ba989.

Hill, N. A. and Häder, D.-P. (1997). A biased random walk model for the trajectories of swimming micro-organisms. Journal of Theoretical Biology, 186(4):503-526. doi:10.1006/jtbi.1997.0421.

Hinkle, J., Fletcher, P. T., and Joshi, S. (2014). Intrinsic polynomials for regression on Riemannian manifolds. Journal of Mathematical Imaging and Vision, 50(1):32-52. doi:10.1007/ s10851-013-0489-5.

Hokimoto, T. and Shimizu, K. (2008). An angular-linear time series model for waveheight prediction. Annals of the Institute of Statistical Mathematics, 60(4):781-800. doi:10.1007/ s10463-008-0207-z.

Hokimoto, T. and Shimizu, K. (2014). A non-homogeneous hidden Markov model for predicting the distribution of sea surface elevation. Journal of Applied Statistics, 41(2):294-319.

Holmquist, B. and Gustafsson, P. (2017). A two-level directional model for dependence in circular data. The Canadian Journal of Statistics, 45(4):461-478. doi:10.1002/cjs.11345.

Holzmann, H., Munk, A., and Stratmann, B. (2004). Identifiability of finite mixtures - with applications to circular distributions. Sankhyā, 66(3):440-449.

Holzmann, H., Munk, A., Suster, M., and Zucchini, W. (2006). Hidden Markov models for circular and linear-circular time series. Environmental and Ecological Statistics, 13(3):325-347. doi: 10.1007/s10651-006-0015-7.

Hornik, K., Feinerer, I., Kober, M., and Buchta, C. (2012). Spherical k-means clustering. Journal of Statistical Software, 50(10):1-22. doi:10.18637/jss.v050.i10.

Hornik, K. and Grün, B. (2013). On conjugate families and Jeffreys priors for von Mises-Fisher distributions. Journal of Statistical Planning and Inference, 143(5):992-999. doi:10.1016/j . jspi.2012.11.003.

Hornik, K. and Grün, B. (2014). movMF: an R package for fitting mixtures of von Mises-Fisher distributions. Journal of Statistical Software, 58(10):1-31. doi:10.18637/jss.v058.i10.

Horwood, J. T. and Poore, A. B. (2014). Gauss von Mises distribution for improved uncertainty realism in space situational awareness. SIAM/ASA Journal on Uncertainty Quantification, 2(1):276304. doi:10.1137/130917296.

Hotz, T. (2013). Extrinsic vs intrinsic means on the circle. In Nielsen, F. and Barbaresco, F. (Eds.), Geometric Science of Information, volume 8085 of Lecture Notes in Computer Science, pp. 433-440, Berlin. Springer. doi:10.1007/978-3-642-40020-9_7.

Hotz, T. and Huckemann, S. (2015). Intrinsic means on the circle: uniqueness, locus and asymptotics. Annals of the Institute of Statistical Mathematics, 67(1):177-193.

Huckemann, S., Hotz, T., and Munk, A. (2010). Intrinsic shape analysis: geodesic PCA for Riemannian manifolds modulo isometric Lie group actions. Statistica Sinica, 20(1):1-58.

Huckemann, S., Kim, K.-R., Munk, A., Rehfeldt, F., Sommerfeld, M., Weickert, J., and Wollnik, C. (2016). The circular SiZer, inferred persistence of shape parameters and application to early stem cell differentiation. Bernoulli, 22(4):2113-2142. doi:10.3150/15-BEJ722.

Huckemann, S. and Ziezold, H. (2006). Principal component analysis for Riemannian manifolds, with an application to triangular shape spaces. Advances in Applied Probability, 38(2):299-319. doi:10.1239/aap/1151337073.

Huckemann, S. F. and Eltzner, B. (2018). Backward nested descriptors asymptotics with inference on stem cell differentiation. The Annals of Statistics, 46(5):1994-2019. doi:10.1214/17-AOS1609.

Hughes, G. (2007). Multivariate and Time Series Models for Circular Data with Applications to Protein Conformational Angles. PhD thesis, University of Leeds.

Humphreys, R. K. and Ruxton, G. D. (2017). Consequences of grouped data for testing for departure from circular uniformity. Behavioral Ecology and Sociobiology, 71(11):167. doi: $10.1007 / \mathrm{s} 00265-017-2393-2$. 
Hundrieser, S., Eltzner, B., and Huckemann, S. F. (2020). Finite sample smeariness of Fréchet means and application to climate. arXiv:2005.02321.

Hung, W.-L., Chang-Chien, S.-J., and Yang, M.-S. (2015). An intuitive clustering algorithm for spherical data with application to extrasolar planets. Journal of Applied Statistics, 42(10):22202232. doi: 10.1080/02664763.2015.1023271.

Hyvärinen, A. (2005). Estimation of non-normalized statistical models by score matching. Journal of Machine Learning Research, 6(Apr):695-709.

Imoto, T., Shimizu, K., and Abe, T. (2019). A cylindrical distribution with heavy-tailed linear part. Japanese Journal of Statistics and Data Science, 2(1):129-154. doi:10.1007/ s42081-019-00031-5.

Irwin, M. E., Cressie, N., and Johannesson, G. (2002). Spatial-temporal nonlinear filtering based on hierarchical statistical models. TEST, 11(2):249-302. doi:10.1007/BF02595708.

Jacimovic, V. and Crnkić, A. (2017). Collective motions of globally coupled oscillators and some probability distributions on circle. Physics Letters A, 381(24):1989-1994. doi:10.1016/j. physleta.2017.04.024.

Jammalamadaka, S. R. and Kozubowski, T. J. (2004). New families of wrapped distributions for modeling skew circular data. Communications in Statistics - Theory and Methods, 33(9):20592074. doi: 10.1081/sta-200026570.

Jammalamadaka, S. R., Meintanis, S., and Verdebout, T. (2020). On new Sobolev tests of uniformity on the circle with extension to the sphere. Bernoulli, 26(3):2226-2252. doi:10.3150/19-BEJ1191.

Jammalamadaka, S. R. and SenGupta, A. (2001). Topics in Circular Statistics, volume 5 of Series on Multivariate Analysis. World Scientific, Singapore. doi:10.1142/4031.

Jammalamadaka, S. R. and Terdik, G. H. (2019). Harmonic analysis and distribution-free inference for spherical distributions. Journal of Multivariate Analysis, 171:436-451. doi:10.1016/j.jmva. 2019.01 .012$.

Jensen, M. H., Mallasto, A., and Sommer, S. (2019). Simulation of conditioned diffusions on the flat torus. In Nielsen, F. and Barbaresco, F. (Eds.), Geometric Science of Information, volume 11712 of Lecture Notes in Computer Science, pp. 685-694, Cham. Springer. doi: 10.1007/978-3-030-26980-7_71.

Jeong, J., Jun, M., and Genton, M. G. (2017). Spherical process models for global spatial statistics. Statistical Science, 32(4):501-513. doi:10.1214/17-STS620.

Johannes, J. and Schwarz, M. (2013). Adaptive circular deconvolution by model selection under unknown error distribution. Bernoulli, 19(5A):1576-1611. doi:10.3150/12-BEJ422.

Johnson, R. A. and Wehrly, T. E. (1977). Measures and models for angular correlation and angularlinear correlation. Journal of the Royal Statistical Society, Series B (Methodological), 39(2):222229. doi:10.1111/j.2517-6161.1977.tb01619.x.

Johnson, R. A. and Wehrly, T. E. (1978). Some angular-linear distributions and related regression models. Journal of the American Statistical Association, 73(363):602-606. doi: 10.1080/01621459.1978.10480062.

Jona-Lasinio, G., Gelfand, A., and Jona-Lasinio, M. (2012). Spatial analysis of wave direction data using wrapped Gaussian processes. The Annals of Applied Statistics, 6(4):1478-1498. doi: 10.1214/12-aoas576.

Jona-Lasinio, G., Gelfand, A. E., and Mastrantonio, G. (2018). Spatial and spatio-temporal circular processes with application to wave directions. In Ley, C. and Verdebout, T. (Eds.), Applied Directional Statistics, Chapman \& Hall/CRC Interdisciplinary Statistics Series, pp. 129-162. CRC Press, Boca Raton.

Jona Lasinio, G., Santoro, M., and Mastrantonio, G. (2020). CircSpaceTime: an R package for spatial and spatio-temporal modelling of circular data. Journal of Statistical Computation and Simulation, 90(7):1315-1345. doi:10.1080/00949655.2020.1725008.

Jones, M. C. and Pewsey, A. (2005). A family of symmetric distributions on the circle. Journal of the American Statistical Association, 100(472):1422-1428. doi:10.1198/016214505000000286. 
Jones, M. C. and Pewsey, A. (2012). Inverse Batschelet distributions for circular data. Biometrics, 68(1):183-193. doi:10.1111/j.1541-0420.2011.01651.x.

Jones, M. C., Pewsey, A., and Kato, S. (2015). On a class of circulas: copulas for circular distributions. Annals of the Institute of Statistical Mathematics, 67(5):843-862.

Jung, S., Dryden, I. L., and Marron, J. S. (2012). Analysis of principal nested spheres. Biometrika, 99(3):551-568. doi:10.1093/biomet/ass022.

Jung, S., Foskey, M., and Marron, J. S. (2011). Principal arc analysis on direct product manifolds. The Annals of Applied Statistics, 5(1):578-603. doi:10.1214/10-aoas370.

Jupp, P. E. (2001). Modifications of the Rayleigh and Bingham tests for uniformity of directions. Journal of Multivariate Analysis, 77(1):1-20. doi:10.1006/jmva.2000.1922.

Jupp, P. E. (2005). Sobolev tests of goodness of fit of distributions on compact Riemannian manifolds. The Annals of Statistics, 33(6):2957-2966. doi:10.1214/009053605000000697.

Jupp, P. E. (2008). Data-driven Sobolev tests of uniformity on compact Riemannian manifolds. The Annals of Statistics, 36(3):1246-1260. doi:10.1214/009053607000000541.

Jupp, P. E. (2009). Data-driven tests of uniformity on product manifolds. Journal of Statistical Planning and Inference, 139(11):3820-3829. doi:10.1016/j.jspi.2009.05.019.

Jupp, P. E. (2015). Copulae on products of compact Riemannian manifolds. Journal of Multivariate Analysis, 140:92-98. doi:10.1016/j.jmva.2015.04.008.

Jupp, P. E. and Kume, A. (2020). Measures of goodness of fit obtained by almost-canonical transformations on Riemannian manifolds. Journal of Multivariate Analysis, 176:104579. doi: 10.1016/j . jmva.2019.104579.

Jupp, P. E. and Mardia, K. V. (1989). A unified view of the theory of directional statistics. International Statistical Review, 57(3):261-294.

Jupp, P. E., Regoli, G., and Azzalini, A. (2016). A general setting for symmetric distributions and their relationship to general distributions. Journal of Multivariate Analysis, 148:107-119. doi:10.1016/j.jmva.2016.02.011.

Kasarapu, P. and Allison, L. (2015). Minimum message length estimation of mixtures of multivariate Gaussian and von Mises-Fisher distributions. Machine Learning, 100(2-3):333-378. doi :10.1007/ s10994-015-5493-0.

Kato, S. (2009). A distribution for a pair of unit vectors generated by Brownian motion. Bernoulli, 15(3):898-921. doi:10.3150/08-bej178.

Kato, S. (2010). A Markov process for circular data. Journal of the Royal Statistical Society, Series B (Statistical Methodology), 72(5):655-672. doi:10.1111/j.1467-9868.2010.00748.x.

Kato, S. and Eguchi, S. (2016). Robust estimation of location and concentration parameters for the von Mises-Fisher distribution. Statistical Papers, 57(1):205-234.

Kato, S. and Jones, M. C. (2010). A family of distributions on the circle with links to, and applications arising from, Möbius transformation. Journal of the American Statistical Association, 105(489):249-262. doi:10.1198/jasa.2009.tm08313.

Kato, S. and Jones, M. C. (2013). An extended family of circular distributions related to wrapped Cauchy distributions via Brownian motion. Bernoulli, 19(1):154-171. doi:10.3150/11-bej397.

Kato, S. and Jones, M. C. (2015). A tractable and interpretable four-parameter family of unimodal distributions on the circle. Biometrika, 102(1):181-190. doi:10.1093/biomet/asu059.

Kato, S. and McCullagh, P. (2020). Some properties of a Cauchy family on the sphere derived from the Möbius transformations. Bernoulli, 266(4):3224-3248. doi:10.3150/20-BEJ1222.

Kato, S. and Pewsey, A. (2015). A Möbius transformation-induced distribution on the torus. Biometrika, 102(2):359-370. doi:10.1093/biomet/asv003.

Kato, S., Pewsey, A., and Jones, M. C. (2018). Circulas from Fourier series. Technical Report 7, School of Mathematics and Statistics, Open University.

Kato, S. and Shimizu, K. (2008). Dependent models for observations which include angular ones. Journal of Statistical Planning and Inference, 138(11):3538-3549. doi:10.1016/j.jspi.2006. 12.009. 
Kato, S., Shimizu, K., and Shieh, G. S. (2008). A circular-circular regression model. Statistica Sinica, 18(2):633-645.

Kaufman, C. G., Ventura, V., and Kass, R. E. (2005). Spline-based non-parametric regression for periodic functions and its application to directional tuning of neurons. Statistics in Medicine, 24(14):2255-2265. doi:10.1002/sim. 2104.

Kendall, D. G., Barden, D., Carne, T. K., and Le, H. (1999). Shape and Shape Theory. Wiley Series in Probability and Statistics. Wiley, Chichester. doi:10.1002/9780470317006.

Kent, J. T. (1975). Discussion of "Statistics of directional data". Journal of the Royal Statistical Society, Series B (Methodological), 37(3):377-378. doi:10.1111/j.2517-6161.1975.tb01550.x.

Kent, J. T., Ganeiber, A. M., and Mardia, K. V. (2018). A new unified approach for the simulation of a wide class of directional distributions. Journal of Computational and Graphical Statistics, 27(2):291-301. doi:10.1080/10618600.2017.1390468.

Kent, J. T., I. Hussein, I., and Jah, M. K. (2016). Directional distributions in tracking of space debris. In 2016 19th International Conference on Information Fusion (FUSION), pp. 2081-2086.

Kent, J. T. and Mardia, K. V. (2009). Principal component analysis for the wrapped normal torus model. In Gusnanto, A., Mardia, K. V., and Fallaize, C. J. (Eds.), LASR 2009 - Statistical Tools for Challenges in Bioinformatics, pp. 39-41, Leeds. Department of Statistics, University of Leeds.

Kent, J. T. and Mardia, K. V. (2015). The winding number for circular data. In Mardia, K. V., Gusnanto, A., Nooney, C., and Voss, J. (Eds.), LASR 2015 - Geometry-Driven Statistics and its Cutting Edge Applications: Celebrating Four Decades of Leeds Statistics Workshops, pp. 47-50, Leeds. Department of Statistics, University of Leeds.

Kent, J. T., Mardia, K. V., and Taylor, C. C. (2008). Modelling strategies for bivariate circular data. In Barber, S., Baxter, P. D., Gusnanto, A., and Mardia, K. V. (Eds.), LASR 2008 - The Art 8 Science of Statistical Bioinformatics, pp. 70-73, Leeds. Department of Statistics, University of Leeds.

Kerkyacharian, G., Pham Ngoc, T. M., and Picard, D. (2011). Localized spherical deconvolution. The Annals of Statistics, 39(2):1042-1068. doi:10.1214/10-aos858.

Kesemen, O., Tezel, Ö., and Özkul, E. (2016). Fuzzy $c$-means clustering algorithm for directional data (FCM4DD). Expert systems with applications, 58:76-82. doi:10.1016/j.eswa.2016.03. 034.

Kim, B., Huckemann, S., Schulz, J., and Jung, S. (2019). Small-sphere distributions for directional data with application to medical imaging. Scandinavian Journal of Statistics, 46(4):1047-1071. doi:10.1111/sjos.12381.

Kim, N. C. and So, H. J. (2018). Directional statistical Gabor features for texture classification. Pattern Recognition Letters, 112:18-26. doi:10.1016/j.patrec.2018.05.010.

Kim, P. T. and Koo, J.-Y. (2002). Optimal spherical deconvolution. Journal of Multivariate Analysis, 80(1):21-42. doi:10.1006/jmva.2000.1968.

Kim, P. T., Koo, J. Y., and Park, H. J. (2004). Sharp minimaxity and spherical deconvolution for super-smooth error distributions. Journal of Multivariate Analysis, 90(2):384-392. doi: $10.1016 / j . j m v a .2003 .08 .004$.

Kim, P. T., Koo, J.-Y., and Pham Ngoc, T. M. (2016). Supersmooth testing on the sphere over analytic classes. Journal of Nonparametric Statistics, 28(1):84-115. doi:10.1080/10485252. 2015.1113284.

Klemelä, J. (1999). Asymptotic minimax risk for the white noise model on the sphere. Scandinavian Journal of Statistics, 26(3):465-473. doi:10.1111/1467-9469.00160.

Klemelä, J. (2000). Estimation of densities and derivatives of densities with directional data. Journal of Multivariate Analysis, 73(1):18-40. doi:10.1006/jmva.1999.1861.

Klemelä, J. (2003). Lower bounds for the asymptotic minimax risk with spherical data. Journal of Statistical Planning and Inference, 113(1):113-136. doi:10.1016/S0378-3758(01)00303-2.

Klugkist, I., Bullens, J., and Postma, A. (2012). Evaluating order-constrained hypotheses for circular data using permutation tests. British Journal of Mathematical and Statistical Psychology, 
65(2):222-236. doi:10.1111/j.2044-8317.2011.02018.x.

Kranstauber, B., Smolla, M., and Scharf, A. K. (2020). move: Visualizing and Analyzing Animal Track Data. R package version 4.0.4. URL: https://CRAN.R-project.org/package=move.

Kueh, A. (2012). Locally adaptive density estimation on the unit sphere using needlets. Constructive Approximation, 36(3):433-458. doi:10.1007/s00365-012-9170-2.

Kume, A. and Sei, T. (2018). On the exact maximum likelihood inference of Fisher-Bingham distributions using an adjusted holonomic gradient method. Statistics and Computing, 28(4):835847.

Kurz, G., Gilitschenski, I., Julier, S. J., and Hanebeck, U. D. (2014). Recursive Bingham filter for directional estimation involving 180 degree symmetry. Journal of Advances in Information Fusion, 9(2):90-105.

Kurz, G., Gilitschenski, I., Pfaff, F., Drude, L., Hanebeck, U. D., Haeb-Umbach, R., and Siegwart, R. Y. (2019). Directional statistics and filtering using libDirectional. Journal of Statistical Software, 89(4):1-31. doi:10.18637/jss.v089.i04.

Lacour, C. and Pham Ngoc, T. M. (2014). Goodness-of-fit test for noisy directional data. Bernoulli, 20(4):2131-2168. doi:10.3150/13-bej553.

Lagona, F. (2016). Regression analysis of correlated circular data based on the multivariate von Mises distribution. Environmental and Ecological Statistics, 23(1):89-113. doi:10.1007/ s10651-015-0330-y.

Lagona, F. (2018). Correlated cylindrical data. In Ley, C. and Verdebout, T. (Eds.), Applied Directional Statistics, Chapman \& Hall/CRC Interdisciplinary Statistics Series, pp. 45-59. CRC Press, Boca Raton.

Lagona, F. and Picone, M. (2011). A latent-class model for clustering incomplete linear and circular data in marine studies. Journal of Data Science, 9(4):585-605.

Lagona, F. and Picone, M. (2012). Model-based clustering of multivariate skew data with circular components and missing values. Journal of Applied Statistics, 39(5):927-945. doi:10.1080/ 02664763.2011 .626850 .

Lagona, F. and Picone, M. (2013). Maximum likelihood estimation of bivariate circular hidden Markov models from incomplete data. Journal of Statistical Computation and Simulation, 83(7):1223-1237. doi:10.1080/00949655.2012.656642.

Lagona, F. and Picone, M. (2016). Model-based segmentation of spatial cylindrical data. Journal of Statistical Computation and Simulation, 86(13):2598-2610.

Lagona, F., Picone, M., and Maruotti, A. (2015a). A hidden Markov model for the analysis of cylindrical time series. Environmetrics, 26(8):534-544. doi:10.1002/env. 2355.

Lagona, F., Picone, M., Maruotti, A., and Cosoli, S. (2015b). A hidden Markov approach to the analysis of space-time environmental data with linear and circular components. Stochastic Environmental Research and Risk Assessment, 29(2):397-409.

Laha, A. K. and Mahesh, K. C. (2015). Robustness of tests for directional mean. Statistics, 49(3):522536.

Laha, A. K., Raja, A. C. P., and Mahesh, K. C. (2019). SB-robust estimation of mean direction for some new circular distributions. Statistical Papers, 60(3):527-552. doi:10.1007/ s00362-016-0853-9.

Landler, L., Ruxton, G. D., and Malkemper, E. P. (2018). Circular data in biology: advice for effectively implementing statistical procedures. Behavioral Ecology and Sociobiology, 72(8):128. doi:10.1007/s00265-018-2538-y.

Landler, L., Ruxton, G. D., and Malkemper, E. P. (2019). Circular statistics meets practical limitations: a simulation-based Rao's spacing test for non-continuous data. Movement Ecology, 7(1):15. doi: 10.1186/s40462-019-0160-x.

Larriba, Y., Rueda, C., Fernández, M. A., and Peddada, S. D. (2020). Order restricted inference in chronobiology. Statistics in Medicine, 39(3):265-278. doi:10.1002/sim. 8397.

Larsen, P. V., Blaesild, P., and Sørensen, M. K. (2002). Improved likelihood ratio tests on the von 
Mises-Fisher distribution. Biometrika, 89(4):947-951. doi:10.1093/biomet/89.4.947.

Le Bihan, N., Chatelain, F., and Manton, J. H. (2016). Isotropic multiple scattering processes on hyperspheres. IEEE Transactions on Information Theory, 62(10):5740-5752. doi:10.1109/TIT. 2015.2508932.

Leguey, I., Bielza, C., and Larrañaga, P. (2019a). Circular Bayesian classifiers using wrapped Cauchy distributions. Data \& Knowledge Engineering, 122:101-115. doi:10.1016/j.datak.2019.05. 005.

Leguey, I., Larrañaga, P., Bielza, C., and Kato, S. (2019b). A circular-linear dependence measure under Johnson-Wehrly distributions and its application in Bayesian networks. Information Sciences, 486:240-253. doi:10.1016/j.ins.2019.01.080.

Lennox, K. P., Dahl, D. B., Vannucci, M., Day, R., and Tsai, J. W. (2010). A Dirichlet process mixture of hidden Markov models for protein structure prediction. The Annals of Applied Statistics, 4(2):916-942. doi:10.1214/09-A0AS296.

Lennox, K. P., Dahl, D. B., Vannucci, M., and Tsai, J. W. (2009). Density estimation for protein conformation angles using a bivariate von Mises distribution and Bayesian nonparametrics. Journal of the American Statistical Association, 104(486):586-596. doi:10.1198/jasa.2009.0024.

Leonenko, N. N. and Ruiz-Medina, M. D. (2018). Increasing domain asymptotics for the first Minkowski functional of spherical random fields. Theory of Probability and Mathematical Statistics, 97:127-149. doi:10.1090/tpms/1053.

Ley, C., Paindaveine, D., and Verdebout, T. (2015). High-dimensional tests for spherical location and spiked covariance. Journal of Multivariate Analysis, 139:79-91. doi:10.1016/j.jmva. 2015. 02.019.

Ley, C., Sabbah, C., and Verdebout, T. (2014). A new concept of quantiles for directional data and the angular Mahalanobis depth. Electronic Journal of Statistics, 8(1):795-816. doi:10.1214/ 14-ejs904.

Ley, C., Swan, Y., Thiam, B., and Verdebout, T. (2013). Optimal R-estimation of a spherical location. Statistica Sinica, 23(1):305-332. doi:10.5705/ss.2011.206.

Ley, C., Swan, Y., and Verdebout, T. (2017). Efficient ANOVA for directional data. Annals of the Institute of Statistical Mathematics, 69(1):39-62. doi:10.1007/s10463-015-0533-x.

Ley, C. and Verdebout, T. (2014a). Local powers of one- and multi-sample tests for the concentration of Fisher-von Mises-Langevin distributions. International Statistical Review, 82(3):440-456.

Ley, C. and Verdebout, T. (2014b). Simple optimal tests for circular reflective symmetry about a specified median direction. Statistica Sinica, 24(3):1319-1339. doi:10.5705/ss.2013.083.

Ley, C. and Verdebout, T. (2017a). Modern Directional Statistics. Chapman \& Hall/CRC Interdisciplinary Statistics Series. CRC Press, Boca Raton. doi:10.1201/9781315119472.

Ley, C. and Verdebout, T. (2017b). Skew-rotationally-symmetric distributions and related efficient inferential procedures. Journal of Multivariate Analysis, 159:67-81. doi:10.1016/j.jmva. 2017. 02.010.

Ley, C. and Verdebout, T. (Eds.) (2018). Applied Directional Statistics. Chapman \& Hall/CRC Interdisciplinary Statistics Series. CRC Press, Boca Raton. doi:10.1201/9781315228570.

Li, L. (2014). Moderate deviations results for a symmetry testing statistic based on the kernel density estimator for directional data. Communications in Statistics - Theory and Methods, 43(14):3007-3018. doi:10.1080/03610926.2012.694545.

Lin, L., St. Thomas, B., Zhu, H., and Dunson, D. B. (2017). Extrinsic local regression on manifoldvalued data. Journal of the American Statistical Association, 112(519):1261-1273. doi:10.1080/ 01621459.2016 .1208615$.

Lin, S.-B. (2019). Nonparametric regression using needlet kernels for spherical data. Journal of Complexity, 50:66-83. doi:10.1016/j.jco.2018.09.003.

Liu, D., Umbach, D. M., Peddada, S. D., Li, L., Crockett, P. W., and Weinberg, C. R. (2004). A random-periods model for expression of cell-cycle genes. Proceedings of the National Academy of Sciences of the United States of America, 101(19):7240-7245. doi:10.1073/pnas.0402285101. 
Liu, R. Y. and Singh, K. (1992). Ordering directional data: concepts of data depth on circles and spheres. The Annals of Statistics, 20(3):1468-1484. doi:10.1214/aos/1176348779.

Loader, C. R. (1996). Local likelihood density estimation. The Annals of Statistics, 24(4):1602-1618. doi:10.1214/aos/1032298287.

Lombard, F., Hawkins, D. M., and Potgieter, C. J. (2017). Sequential rank CUSUM charts for angular data. Computational Statistics ES Data Analysis, 105:268-279. doi:10.1016/j.csda. 2016.08 .001$.

Lombard, F. and Maxwell, R. K. (2012). A cusum procedure to detect deviations from uniformity in angular data. Journal of Applied Statistics, 39(9):1871-1880. doi:10.1080/02664763.2012. 683857.

López-Cruz, P. L., Bielza, C., and Larrañaga, P. (2015). Directional naive Bayes classifiers. Pattern Analysis and Applications, 18(2):225-246. doi:10.1007/s10044-013-0340-z.

Lu, Y., Corander, J., and Yang, Z. (2019). Doubly stochastic neighbor embedding on spheres. Pattern Recognition Letters, 128:100-106. doi:10.1016/j.patrec.2019.08.026.

Lund, U. (1999). Cluster analysis for directional data. Communications in Statistics - Simulation and Computation, 28(4):1001-1009. doi:10.1080/03610919908813589.

Lund, U. (2002). Tree-based regression for a circular response. Communications in Statistics Theory and Methods, 31(9):1549-1560. doi:10.1081/sta-120013011.

Lunga, D. and Ersoy, O. (2013). Spherical stochastic neighbor embedding of hyperspectral data. IEEE Transactions on Geoscience and Remote Sensing, 51(2):857-871. doi :10.1109/tgrs. 2012 . 2205004.

van der Maaten, L. J. P. and Hinton, G. E. (2008). Visualizing high-dimensional data using t-SNE. Journal of Machine Learning Research, 9(Nov):2579-2605.

Mahmood, E. A., Rana, S., Midi, H., and Hussin, A. G. (2017). Detection of outliers in univariate circular data using robust circular distance. Journal of Modern Applied Statistical Methods, 16(2):22. doi:10.22237/jmasm/1509495720.

Maitra, R. and Ramler, I. P. (2010). A $k$-mean-directions algorithm for fast clustering of data on the sphere. Journal of Computational and Graphical Statistics, 19(2):377-396. doi:10.1198/jcgs . 2009.08155.

Maksimov, V. M. (1967). Necessary and sufficient statistics for the family of shifts of probability distributions on continuous bicompact groups (in Russian). Theoria Verojatna, 12(2):307-321.

Mardia, K. (2018). A new estimation methodology for standard directional distributions. In 2018 21st International Conference on Information Fusion (FUSION), pp. 724-729, New York. IEEE.

Mardia, K. V. (1972). Statistics of Directional Data. Probability and Mathematical Statistics. Academic Press, London.

Mardia, K. V. (1975). Statistics of directional data. Journal of the Royal Statistical Society, Series B (Methodological), 37(3):349-393. doi:10.1111/j.2517-6161.1975.tb01550.x.

Mardia, K. V. (2010). Bayesian analysis for bivariate von Mises distributions. Journal of Applied Statistics, 37(3):515-528. doi:10.1080/02664760903551267.

Mardia, K. V., Foldager, J. I., and Frellsen, J. (2018). Directional statistics in protein bioinformatics. In Ley, C. and Verdebout, T. (Eds.), Applied Directional Statistics, Chapman \& Hall/CRC Interdisciplinary Statistics Series, pp. 1-23. CRC Press, Boca Raton.

Mardia, K. V. and Frellsen, J. (2012). Statistics of bivariate von Mises distributions. In Hamelryck, T., Mardia, K., and Ferkinghoff-Borg, J. (Eds.), Bayesian Methods in Structural Bioinformatics, Statistics for Biology and Health, pp. 159-178. Springer, Berlin. doi:10.1007/ 978-3-642-27225-7_6.

Mardia, K. V., Hughes, G., Taylor, C. C., and Singh, H. (2008). A multivariate von Mises distribution with applications to bioinformatics. The Canadian Journal of Statistics, 36(1):99-109. doi: $10.1002 /$ cjs. 5550360110 .

Mardia, K. V. and Jupp, P. E. (1999). Directional Statistics. Wiley Series in Probability and Statistics. Wiley, Chichester. doi:10.1002/0471667196.ess7086. 
Mardia, K. V., Kent, J. T., and Laha, A. K. (2016). Score matching estimators for directional distributions. arXiv:1604.08470.

Mardia, K. V., Kent, J. T., Zhang, Z., Taylor, C. C., and Hamelryck, T. (2012). Mixtures of concentrated multivariate sine distributions with applications to bioinformatics. Journal of Applied Statistics, 39(11):2475-2492. doi:10.1080/02664763.2012.719221.

Mardia, K. V. and Patrangenaru, V. (2005). Directions and projective shapes. The Annals of Statistics, 33(4):1666-1699. doi:10.1214/009053605000000273.

Mardia, K. V. and Sutton, T. W. (1978). A model for cylindrical variables with applications. Journal of the Royal Statistical Society, Series B (Methodological), 40(2):229-233. doi:10.1111/ j.2517-6161.1978.tb01668.x.

Mardia, K. V., Taylor, C. C., and Subramaniam, G. K. (2007). Protein bioinformatics and mixtures of bivariate von Mises distributions for angular data. Biometrics, 63(2):505-512. doi:10.1111/ j.1541-0420.2006.00682.x.

Mardia, K. V. and Voss, J. (2014). Some fundamental properties of a multivariate von Mises distribution. Communications in Statistics - Theory and Methods, 43(6):1132-1144. doi:10. 1080/03610926.2012.670353.

Marinucci, D. and Peccati, G. (2011). Random Fields on the Sphere. London Mathematical Society Lecture Note Series. Cambridge University Press, Cambridge.

Marinucci, D., Pietrobon, D., Balbi, A., Baldi, P., Cabella, P., Kerkyacharian, G., Natoli, P., Picard, D., and Vittorio, N. (2008). Spherical needlets for cosmic microwave background data analysis. Monthly Notices of the Royal Astronomical Society, 383(2):539-545. doi:10.1111/j.1365-2966. $2007.12550 . \mathrm{x}$.

Marron, J. S. and Alonso, A. M. (2014). Overview of object oriented data analysis. Biometrical Journal, 56(5):732-753. doi:10.1002/bimj . 201300072.

Maruotti, A. (2016). Analyzing longitudinal circular data by projected normal models: a semiparametric approach based on finite mixture models. Environmental and Ecological Statistics, 23(2):257-277. doi:10.1007/s10651-015-0338-3.

Maruotti, A., Punzo, A., Mastrantonio, G., and Lagona, F. (2016). A time-dependent extension of the projected normal regression model for longitudinal circular data based on a hidden Markov heterogeneity structure. Stochastic Environmental Research and Risk Assessment, 30(6):17251740. doi:10.1007/s00477-015-1183-5.

Mash'al, M. and Hosseini, R. (2015). K-means ++ for mixtures of von Mises-Fisher distributions. In 2015 7th Conference on Information and Knowledge Technology (IKT), pp. 1-6, New York. IEEE. doi:10.1109/ikt.2015.7288786.

Mastrantonio, G. (2018). The joint projected normal and skew-normal: a distribution for polycylindrical data. Journal of Multivariate Analysis, 165:14-26. doi:10.1016/j.jmva.2017.11. 006.

Mastrantonio, G. and Calise, G. (2016). Hidden Markov model for discrete circular-linear wind data time series. Journal of Statistical Computation and Simulation, 86(13):2611-2624. doi: 10.1080/00949655.2016.1142544.

Mastrantonio, G., Gelfand, A. E., and Jona Lasinio, G. (2016a). The wrapped skew Gaussian process for analyzing spatio-temporal data. Stochastic Environmental Research and Risk Assessment, 30(8):2231-2242. doi:10.1007/s00477-015-1163-9.

Mastrantonio, G., Jona Lasinio, G., and Gelfand, A. E. (2016b). Spatio-temporal circular models with non-separable covariance structure. TEST, 25(2):331-350. doi:10.1007/ s11749-015-0458-y.

Mastrantonio, G., Jona Lasinio, G., Maruotti, A., and Calise, G. (2019). Invariance properties and statistical inference for circular data. Statistica Sinica, 29(1):67-80. doi:10.5705/ss.202016. 0067.

Mastrantonio, G., Maruotti, A., and Jona Lasinio, G. (2015). Bayesian hidden Markov modelling using circular-linear general projected normal distribution. Environmetrics, 26(2):145-158. doi: 
10.1002/env. 2326.

Mazumder, S. and Bhattacharya, S. (2016). Bayesian nonparametric dynamic state space modeling with circular latent states. Journal of Statistical Theory and Practice, 10(1):154-178. doi: 10.1080/15598608.2015.1100562.

Mazumder, S. and Bhattacharya, S. (2017). Nonparametric dynamic state space modeling of observed circular time series with circular latent states: a Bayesian perspective. Journal of Statistical Theory and Practice, 11(4):693-718. doi:10.1080/15598608.2017.1305922.

McClintock, B. T., King, R., Thomas, L., Matthiopoulos, J., McConnell, B. J., and Morales, J. M. (2012). A general discrete-time modeling framework for animal movement using multistate random walks. Ecological Monographs, 82(3):335-349. doi:10.1890/11-0326.1.

McCullagh, P. (1996). Möbius transformation and Cauchy parameter estimation. The Annals of Statistics, 24(2):787-808. doi:10.1214/aos/1032894465.

McMillan, G. P., Hanson, T. E., Saunders, G., and Gallun, F. J. (2013). A two-component circular regression model for repeated measures auditory localization data. Journal of the Royal Statistical Society, Series C (Applied Statistics), 62(4):515-534. doi:10.1111/rssc.12004.

McVinish, R. and Mengersen, K. (2008). Semiparametric Bayesian circular statistics. Computational Statistics \& Data Analysis, 52(10):4722-4730. doi:10.1016/j.csda.2008.03.016.

Meilán-Vila, A., Francisco-Fernández, M., Crujeiras, R. M., and Panzera, A. (2020). Nonparametric multivariate regression estimation for circular responses. arXiv:2001.1031\%.

Meintanis, S. and Verdebout, T. (2019). Le Cam maximin tests for symmetry of circular data based on the characteristic function. Statistica Sinica, 29(3):1301-1320. doi:10.5705/ss .202016.0016.

Michelot, T., Langrock, R., Patterson, T., and McClintock, B. (2016). moveHMM: an R package for the statistical modelling of animal movement data using hidden Markov models. Methods in Ecology and Evolution, 7(11):1308-1315. doi:10.1111/2041-210X.12578.

Miolane, N., Le Brigant, A., Mathe, J., Hou, B., Guigui, N., Thanwerdas, Y., Heyder, S., Peltre, O., Koep, N., Zaatiti, H., Hajri, H., Cabanes, Y., Gerald, T., Chauchat, P., Shewmake, C., Kainz, B., Donnat, C., Holmes, S., and Pennec, X. (2020). geomstats: A Python package for Riemannian geometry in machine learning. arXiv:2004.0466\%.

Miyata, Y., Shiohama, T., and Abe, T. (2019). Estimation of finite mixture models of skewsymmetric circular distributions. Metrika. doi:10.1007/s00184-019-00756-z.

Modlin, D., Fuentes, M., and Reich, B. (2012). Circular conditional autoregressive modeling of vector fields. Environmetrics, 23(1):46-53. doi:10.1002/env.1133.

Moghimbeygi, M. and Golalizadeh, M. (2020). Spherical logistic distribution. Communications in Mathematics and Statistics, 8(2):151-166. doi:10.1007/s40304-018-00171-2.

Monbet, V. (2020). NHMSAR: Non-Homogeneous Markov Switching Autoregressive Models. R package version 1.17. URL: https://CRAN.R-project.org/package=NHMSAR.

Monnier, J.-B. (2011). Non-parametric regression on the hypersphere with uniform design. TEST, 20(2):412-446. doi:10.1007/s11749-011-0233-7.

Montanari, A. and Calò, D. G. (2013). Model-based clustering of probability density functions. Advances in Data Analysis and Classification, 7(3):301-319. doi:10.1007/s11634-013-0140-8.

Mooney, J. A., Helms, P. J., and Jolliffe, I. T. (2003). Fitting mixtures of von Mises distributions: a case study involving sudden infant death syndrome. Computational Statistics 8 Data Analysis, 41(3-4):505-513. doi:10.1016/s0167-9473(02)00181-0.

Morales, J. M., Haydon, D. T., Frair, J., Holsinger, K. E., and Fryxell, J. M. (2004). Extracting more out of relocation data: building movement models as mixtures of random walks. Ecology, 85(9):2436-2445. doi:10.1890/03-0269.

Morphet, W. J. and Symanzik, J. (2010). The circular dataimage, a graph for high-resolution circular-spatial data. International Journal of Digital Earth, 3(1):47-71. doi:10.1080/ 17538940903277657.

Mu, Y., Nguyen, P. H., and Stock, G. (2005). Energy landscape of a small peptide revealed by dihedral angle principal component analysis. Proteins: Structure, Function, and Bioinformatics, 
58(1):45-52. doi:10.1002/prot. 20310.

Mulder, K., Jongsma, P., and Klugkist, I. (2020a). Bayesian inference for mixtures of von Mises distributions using reversible jump MCMC sampler. Journal of Statistical Computation and Simulation, 90(9):1539-1556. doi:10.1080/00949655.2020.1740997.

Mulder, K. and Klugkist, I. (2017). Bayesian estimation and hypothesis tests for a circular generalized linear model. Journal of Mathematical Psychology, 80:4-14. doi:10.1016/j.jmp.2017.07. 001.

Mulder, K., Klugkist, I., van Renswoude, D., and Visser, I. (2020b). Mixtures of peaked power Batschelet distributions for circular data with application to saccade directions. Journal of Mathematical Psychology, 95:102309. doi:10.1016/j.jmp.2019.102309.

Mulder, K. T. and Klugkist, I. (2021). Bayesian tests for circular uniformity. Journal of Statistical Planning and Inference, 211:315-325. doi:10.1016/j.jspi.2020.06.002.

Munro, M. A. and Blenkinsop, T. G. (2012). MARD - A moving average rose diagram application for the geosciences. Computers \&S Geosciences, 49:112-120. doi:10.1016/j . cageo. 2012.07.012.

Mushkudiani, N. A. (2002). Small nonparametric tolerance regions for directional data. Journal of Statistical Planning and Inference, 100(1):67-80. doi:10.1016/S0378-3758(01)00093-3.

Narcowich, F. J., Petrushev, P., and Ward, J. D. (2006). Localized tight frames on spheres. SIAM Journal on Mathematical Analysis, 38(2):574-594. doi:10.1137/040614359.

Navarro, A. K. W., Frellsen, J., and Turner, R. E. (2017). The multivariate generalised von Mises distribution: inference and applications. In Proceedings of the Thirty-First AAAI Conference on Artificial Intelligence (AAAI-17), pp. 2394-2400, San Francisco. Association for the Advancement of Artificial Intelligence.

Nicosia, A., Duchesne, T., Rivest, L.-P., and Fortin, D. (2017). A general hidden state random walk model for animal movement. Computational Statistics \& Data Analysis, 105:76-95. doi: $10.1016 / j$.csda. 2016.07.009.

Nodehi, A., Golalizadeh, M., and Heydari, A. (2015). Dihedral angles principal geodesic analysis using nonlinear statistics. Journal of Applied Statistics, 42(9):1962-1972. doi:10.1080/02664763. 2015.1014892.

Núñez-Antonio, G. and Geneyro, E. (2020). A multivariate projected gamma model for directional data. Communications in Statistics: Case Studies, Data Analysis and Applications, to appear. doi:10.1080/03610918.2019.1612910.

Núñez-Antonio, G. and Gutiérrez-Peña, E. (2005a). A Bayesian analysis of directional data using the projected normal distribution. Journal of Applied Statistics, 32(10):995-1001. doi:10.1080/ 02664760500164886.

Núñez-Antonio, G. and Gutiérrez-Peña, E. (2005b). A Bayesian analysis of directional data using the von Mises-Fisher distribution. Communications in Statistics - Simulation and Computation, 34(4):989-999. doi:10.1080/03610910500308495.

Núñez-Antonio, G. and Gutiérrez-Peña, E. (2014). A Bayesian model for longitudinal circular data based on the projected normal distribution. Computational Statistics 8 Data Analysis, 71:506519. doi:10.1016/j.csda.2012.07.025.

Núñez-Antonio, G., Gutiérrez-Peña, E., and Escarela, G. (2011). A Bayesian regression model for circular data based on the projected normal distribution. Statistical Modelling, 11(3):185-201. doi:10.1177/1471082x1001100301.

Núñez-Antonio, G., Mendoza, M., Contreras-Cristán, A., Gutiérrez-Peña, E., and Mendoza, E. (2018). Bayesian nonparametric inference for the overlap of daily animal activity patterns. Environmental and Ecological Statistics, 25(4):471-494. doi:10.1007/s10651-018-0414-6.

Oba, S., Kato, K., and Ishii, S. (2005). Multi-scale clustering for gene expression profiling data. In Fifth IEEE Symposium on Bioinformatics and Bioengineering (BIBE '05), pp. 210-217. doi: 10.1109/BIBE. 2005.41.

Oliveira, M., Crujeiras, R. M., and Rodríguez-Casal, A. (2012). A plug-in rule for bandwidth selection in circular density estimation. Computational Statistics $\&$ Data Analysis, 56(12):3898- 
3908. doi:10.1016/j.csda.2012.05.021.

Oliveira, M., Crujeiras, R. M., and Rodríguez-Casal, A. (2014). CircSiZer: an exploratory tool for circular data. Environmental and Ecological Statistics, 21(1):143-159. doi:10.1007/ s10651-013-0249-0.

Otieno, S. B. and Anderson-Cook, C. M. (2012). Design and analysis of experiments for directional data. In Hinkelmann, K. (Ed.), Design and Analysis of Experiments, Wiley Series in Probability and Statistic, pp. 501-532. Wiley, Hoboken. doi:10.1002/9781118147634.ch15.

Oualkacha, K. and Rivest, L.-P. (2009). A new statistical model for random unit vectors. Journal of Multivariate Analysis, 100(1):70-80. doi:10.1016/j.jmva.2008.03.004.

Paindaveine, D. and Verdebout, T. (2015). Optimal rank-based tests for the location parameter of a rotationally symmetric distribution on the hypersphere. In Hallin, M., Mason, D., Pfeifer, D., and Steinebach, J. (Eds.), Mathematical Statistics and Limit Theorems, pp. 249-269. Springer, Cham. doi:10.1007/978-3-319-12442-1_14.

Paindaveine, D. and Verdebout, T. (2016). On high-dimensional sign tests. Bernoulli, 22(3):17451769. doi:10.3150/15-bej710.

Paindaveine, D. and Verdebout, T. (2017). Inference on the mode of weak directional signals: a Le Cam perspective on hypothesis testing near singularities. The Annals of Statistics, 45(2):800-832. doi:10.1214/16-aos1468.

Paindaveine, D. and Verdebout, T. (2020). Inference for spherical location under high concentration. The Annals of Statistics, to appear.

Paine, P. J., Preston, S. P., Tsagris, M., and Wood, A. T. A. (2018). An elliptically symmetric angular Gaussian distribution. Statistics and Computing, 28(3):689-697. doi:10.1007/ s11222-017-9756-4.

Paine, P. J., Preston, S. P., Tsagris, M., and Wood, A. T. A. (2020). Spherical regression models with general covariates and anisotropic errors. Statistics and Computing, 30(1):153-165. doi: 10.1007/s11222-019-09872-2.

Paluszewski, M. and Hamelryck, T. (2010). Mocapy ++- a toolkit for inference and learning in dynamic Bayesian networks. BMC Bioinformatics, 11(126):1-6. doi:10.1186/1471-2105-11-126.

Panaretos, V. M., Pham, T., and Yao, Z. (2014). Principal flows. Journal of the American Statistical Association, 109(505):424-436. doi:10.1080/01621459.2013.849199.

Pandolfo, G., D'Ambrosio, A., and Porzio, G. C. (2018a). A note on depth-based classification of circular data. Electronic Journal of Applied Statistical Analysis, 11(2):447-462. doi:10.1285/ i20705948v11n2p447.

Pandolfo, G., Paindaveine, D., and Porzio, G. C. (2018b). Distance-based depths for directional data. The Canadian Journal of Statistics, 46(4):593-609. doi:10.1002/cjs.11479.

Pardo, A., Real, E., Krishnaswamy, V., López-Higuera, J. M., Pogue, B. W., and Conde, O. M. (2017). Directional kernel density estimation for classification of breast tissue spectra. IEEE Transactions on Medical Imaging, 36(1):64-73. doi:10.1109/tmi.2016.2593948.

Park, H. S. (2012). Asymptotic behavior of the kernel density estimator from a geometric viewpoint. Communications in Statistics - Simulation and Computation, 41(19):3479-3496. doi:10.1080/ 03610926.2011 .585009$.

Park, H. S. (2013). Comparison of relative efficiency of kernel density estimator with the exponential map. Journal of the Korean Statistical Society, 42(2):267-275. doi:10.1016/j.jkss.2012.08. 007.

Peel, D., Whiten, W. J., and McLachlan, G. J. (2001). Fitting mixtures of Kent distributions to aid in joint set identification. Journal of the American Statistical Association, 96(453):56-63. doi:10.1198/016214501750332974.

Pelletier, B. (2005). Kernel density estimation on Riemannian manifolds. Statistics \& Probability Letters, 73(3):297-304. doi:10.1016/j.spl.2005.04.004.

Pennec, X. (2018). Barycentric subspace analysis on manifolds. The Annals of Statistics, 46(6A):2711-2746. doi:10.1214/17-aos1636. 
Pertsemlidis, A., Zelinka, J., Fondon, J. W., Henderson, R. K., and Otwinowski, Z. (2005). Bayesian statistical studies of the Ramachandran distribution. Statistical Applications in Genetics and Molecular Biology, 4(1). doi:10.2202/1544-6115.1165.

Pewsey, A. (2000). The wrapped skew-normal distribution on the circle. Communications in Statistics - Theory and Methods, 29(11):2459-2472. doi:10.1080/03610920008832616.

Pewsey, A. (2002). Testing circular symmetry. The Canadian Journal of Statistics, 30(4):591-600. doi: $10.2307 / 3316098$.

Pewsey, A. (2004a). The large-sample joint distribution of key circular statistics. Metrika, 60(1):2532. doi:10.1007/s001840300294.

Pewsey, A. (2004b). Testing for circular reflective symmetry about a known median axis. Journal of Applied Statistics, 31(5):575-585. doi:10.1080/02664760410001681828.

Pewsey, A. (2006). Modelling asymmetrically distributed circular data using the wrapped skewnormal distribution. Environmental and Ecological Statistics, 13(3):257-269. doi:10.1007/ s10651-005-0010-4.

Pewsey, A. (2008). The wrapped stable family of distributions as a flexible model for circular data. Computational Statistics \& Data Analysis, 52(3):1516-1523. doi:10.1016/j . csda.2007.04.017.

Pewsey, A. (2018). Applied directional statistics with R: an overview. In Ley, C. and Verdebout, T. (Eds.), Applied Directional Statistics, Chapman \& Hall/CRC Interdisciplinary Statistics Series, pp. 277-290. CRC Press, Boca Raton.

Pewsey, A. and Jones, M. C. (2005). Discrimination between the von Mises and wrapped normal distributions: just how big does the sample size have to be? Statistics, 39(2):81-89. doi: 10.1080/02331880500031597.

Pewsey, A. and Kato, S. (2016). Parametric bootstrap goodness-of-fit testing for Wehrly-Johnson bivariate circular distributions. Statistics and Computing, 26(6):1307-1317. doi:10.1007/ s11222-015-9605-2.

Pewsey, A., Lewis, T., and Jones, M. C. (2007). The wrapped $t$ family of circular distributions. Australian $\&$ New Zealand Journal of Statistics, 49(1):79-91. doi:10.1111/j.1467-842x.2006. 00465.x.

Pewsey, A., Neuhäuser, M., and Ruxton, G. D. (2013). Circular Statistics in R. Oxford University Press, Oxford.

Pham Ngoc, T. M. (2019). Adaptive optimal kernel density estimation for directional data. Journal of Multivariate Analysis, 173:248-267. doi:10.1016/j.jmva.2019.02.009.

Pitt, M. K. and Shephard, N. (1999). Filtering via simulation: auxiliary particle filters. Journal of the American Statistical Association, 94(446):590-599. doi:10.2307/2670179.

Pizer, S. M., Jung, S., Goswami, D., Vicory, J., Zhao, X., Chaudhuri, R., Damon, J. N., Huckemann, S., and Marron, J. S. (2013). Nested sphere statistics of skeletal models. In Breuß, M., Bruckstein, A., and Maragos, P. (Eds.), Innovations for Shape Analysis, Mathematics and Visualization, pp. 93-115. Springer, Berlin. doi:10.1007/978-3-642-34141-0_5.

Polsen, O. and Taylor, C. C. (2015). Parametric circular-circular regression and diagnostic analysis. In Dryden, I. L. and Kent, J. T. (Eds.), Geometry Driven Statistics, Wiley Series in Probability and Statistics, pp. 115-128. Wiley, Chichester. doi:10.1002/9781118866641.ch5.

Porcu, E., Bevilacqua, M., and Genton, M. G. (2016). Spatio-temporal covariance and crosscovariance functions of the great circle distance on a sphere. Journal of the American Statistical Association, 111(514):888-898. doi:10.1080/01621459.2015.1072541.

Porcu, E., Furrer, R., and Nychka, D. (2020). 30 years of space-time covariance functions. WIREs Computational Statistics, to appear:e1512. doi:10.1002/wics.1512.

Presnell, B., Morrison, S. P., and Littell, R. C. (1998). Projected multivariate linear models for directional data. Journal of the American Statistical Association, 93(443):1068-1077. doi:10. $2307 / 2669850$.

Pycke, J.-R. (2007). A decomposition for invariant tests of uniformity on the sphere. Proceedings of the American Mathematical Society, 135(9):2983-2993. doi:10.1090/s0002-9939-07-08804-1. 
Pycke, J.-R. (2010). Some tests for uniformity of circular distributions powerful against multimodal alternatives. The Canadian Journal of Statistics, 38(1):80-96. doi:10.1002/cjs.10048.

Qin, X., Zhang, J.-S., and Yan, X.-D. (2011). A nonparametric circular-linear multivariate regression model with a rule-of-thumb bandwidth selector. Computers and Mathematics with Applications, 62(8):3048-3055. doi:10.1016/j.camwa.2011.08.016.

Qiu, X., Wu, S., and Wu, H. (2015). A new information criterion based on Langevin mixture distribution for clustering circular data with application to time course genomic data. Statistica Sinica, 25(4):1459-1476. doi:10.5705/ss.2013.030.

R Core Team (2020). R: A Language and Environment for Statistical Computing. R Foundation for Statistical Computing. URL: https://www.R-project.org/.

Rakhimberdiev, E., Saveliev, A., Piersma, T., and Karagicheva, J. (2017). FLightR: an R package for reconstructing animal paths from solar geolocation loggers. Methods in Ecology and Evolution, 8(11):1482-1487. doi:10.1111/2041-210X.12765.

Ranalli, M., Lagona, F., Picone, M., and Zambianchi, E. (2018). Segmentation of sea current fields by cylindrical hidden Markov models: a composite likelihood approach. Journal of the Royal Statistical Society, Series C (Applied Statistics), 67(3):575-598. doi:10.1111/rssc.12240.

Rayleigh, Lord. (1919). On the problem of random vibrations, and of random flights in one, two, or three dimensions. The London, Edinburgh, and Dublin Philosophical Magazine and Journal of Science, 37(220):321-347. doi:10.1080/14786440408635894.

Reed, W. J. and Pewsey, A. (2009). Two nested families of skew-symmetric circular distributions. TEST, 18(3):516-528. doi:10.1007/s11749-008-0111-0.

Riccardi, L., Nguyen, P. H., and Stock, G. (2009). Free-energy landscape of RNA hairpins constructed via dihedral angle principal component analysis. The Journal of Physical Chemistry B, 113(52):16660-16668. doi:10.1021/jp9076036.

Rivest, L.-P. (1997). A decentred predictor for circular-circular regression. Biometrika, 84(3):717726. doi:10.1093/biomet/84.3.717.

Rivest, L.-P. (1999). Some linear model techniques for analyzing small circle spherical data. The Canadian Journal of Statistics, 27(3):623-638. doi:10.2307/3316117.

Rivest, L.-P., Duchesne, T., Nicosia, A., and Fortin, D. (2016). A general angular regression model for the analysis of data on animal movement in ecology. Journal of the Royal Statistical Society, Series C (Applied Statistics), 65(3):445-463. doi:10.1111/rssc.12124.

Rivest, L.-P. and Kato, S. (2019). A random-effects model for clustered circular data. The Canadian Journal of Statistics, 47(4):712-728. doi:10.1002/cjs.11520.

Rivest, L.-P. and Oualkacha, K. (2018). On modeling of SE(3) objects. In Ley, C. and Verdebout, T. (Eds.), Applied Directional Statistics, Chapman \& Hall/CRC Interdisciplinary Statistics Series, pp. 111-127. CRC Press, Boca Raton.

Rodgers, J. L., Beasley, W. H., and Schuelke, M. (2014). Graphical data analysis on the circle: wraparound time series plots for (interrupted) time series designs. Multivariate Behavioral Research, 49(6):571-580. doi:10.1080/00273171.2014.946589.

Rodríguez, C. E., Núñez-Antonio, G., and Escarela, G. (2020). A Bayesian mixture model for clustering circular data. Computational Statistics \& Data Analysis, 143:106842. doi:10.1016/ j.csda.2019.106842.

Rodriguez-Lujan, L., Bielza, C., and Larrañaga, P. (2015). Regularized multivariate von mises distribution. In Puerta, J. M., Gámez, J. A., Dorronsoro, B., Barrenechea, E., Troncoso, A., Baruque, B., and Galar, M. (Eds.), Advances in Artificial Intelligence, volume 9422 of Lecture Notes in Computer Science, pp. 25-35, Cham. Springer. doi:10.1007/978-3-319-24598-0_3.

Rodriguez-Lujan, L., Bielza, C., and Larrañaga, P. (2017). Frobenius norm regularization for the multivariate von Mises distribution. International Journal of Intelligent Systems, 32(2):153-176. doi:10.1002/int.21834.

Rosenthal, M., Wu, W., Klassen, E., and Srivastava, A. (2014). Spherical regression models using projective linear transformations. Journal of the American Statistical Association, 109(508):1615- 
1624. doi:10.1080/01621459.2014.892881.

Rothman, E. D. (1972). Tests for uniformity of a circular distribution. Sankhyā, Series A, 34(1):2332.

Roy, A., Pal, A., and Garain, U. (2017). JCLMM: A finite mixture model for clustering of circularlinear data and its application to psoriatic plaque segmentation. Pattern Recognition, 66:160-173. doi: $10.1016 / j \cdot$ patcog. 2016.12.016.

Rueda, C., Fernández, M. A., Barragán, S., Mardia, K. V., and Peddada, S. D. (2016). Circular piecewise regression with applications to cell-cycle data. Biometrics, 72(4):1266-1274. doi: 10.1111/biom. 12512 .

Rueda, C., Fernández, M. A., Barragán, S., and Peddada, S. D. (2015). Some advances in constrained inference for ordered circular parameters in oscillatory systems. In Dryden, I. L. and Kent, J. T. (Eds.), Geometry Driven Statistics, Wiley Series in Probability and Statistics, pp. 97-114. Wiley, Chichester. doi:10.1002/9781118866641.ch4.

Rueda, C., Fernández, M. A., and Peddada, S. D. (2009). Estimation of parameters subject to order restrictions on a circle with application to estimation of phase angles of cell cycle genes. Journal of the American Statistical Association, 104(485):338-347. doi:10.1198/jasa.2009.0120.

Rumcheva, P. and Presnell, B. (2017). An improved test of equality of mean directions for the Langevin-von Mises-Fisher distribution. Australian 83 New Zealand Journal of Statistics, 59(1):119-135. doi:10.1111/anzs.12183.

Sadikon, N. H., Ibrahim, A. I. N., Mohamed, I., and Shimizu, K. (2019). A new test of discordancy in cylindrical data. Communications in Statistics - Simulation and Computation, 48(8):2512-2522. doi:10.1080/03610918.2018.1458131.

Sahoo, I., Guinness, J., and Reich, B. J. (2019). A test for isotropy on a sphere using spherical harmonic functions. Statistica Sinica, 29(3):1253-1276. doi:10.5705/ss.202017.0475.

Salah, A. and Nadif, M. (2017). Social regularized von Mises-Fisher mixture model for item recommendation. Data Mining and Knowledge Discovery, 31(5, SI):1218-1241. doi:10.1007/ s10618-017-0499-9.

Salah, A. and Nadif, M. (2019). Directional co-clustering. Advances in Data Analysis and Classifcation, 13(3):591-620. doi:10.1007/s11634-018-0323-4.

Sargsyan, K., Hua, Y. H., and Lim, C. (2015). Clustangles: an open library for clustering angular data. Journal of Chemical Information and Modeling, 55(8):1517-1520. doi:10.1021/acs .jcim. $5 b 00316$.

Sargsyan, K., Wright, J., and Lim, C. (2012). GeoPCA: a new tool for multivariate analysis of dihedral angles based on principal component geodesics. Nucleic Acids Research, 40(3):e25-e25. doi:10.1093/nar/gkv1000.

Sarma, Y. R. and Jammalamadaka, S. R. (1993). Circular regression. In Matsusita, K., Puri, M. L., and Hayakawa, T. (Eds.), Statistical Science and Data Analysis, pp. 109-128, Utrecht. VSP.

Sau, M. F. and Rodriguez, D. (2018). Minimum distance method for directional data and outlier detection. Advances in Data Analysis and Classification, 12(3):587-603. doi:10.1007/ s11634-017-0287-9.

Saw, J. G. (1983). Dependent unit vectors. Biometrika, 70(3):665-671. doi:10.1093/biomet/70. 3.665 .

Scealy, J. L. and Welsh, A. H. (2011). Regression for compositional data by using distributions defined on the hypersphere. Journal of the Royal Statistical Society, Series B (Statistical Methodology), 73(3):351-375. doi:10.1111/j.1467-9868.2010.00766.x.

Scealy, J. L. and Welsh, A. H. (2014a). Colours and cocktails: compositional data analysis: 2013 Lancaster lecture. Australian \& New Zealand Journal of Statistics, 56(2):145-169. doi:10.1111/ anzs. 12073.

Scealy, J. L. and Welsh, A. H. (2014b). Fitting Kent models to compositional data with small concentration. Statistics and Computing, 24(2):165-179. doi:10.1007/s11222-012-9361-5.

Scealy, J. L. and Welsh, A. H. (2017). A directional mixed effects model for compositional 
expenditure data. Journal of the American Statistical Association, 112(517):24-36. doi: 10.1080/01621459.2016.1189336.

Scealy, J. L. and Wood, A. T. A. (2019). Scaled von Mises-Fisher distributions and regression models for paleomagnetic directional data. Journal of the American Statistical Association, 114(528):1547-1560. doi:10.1080/01621459.2019.1585249.

Schlather, M., Malinowski, A., Menck, P. J., Oesting, M., and Strokorb, K. (2015). Analysis, simulation and prediction of multivariate random fields with package RandomFields. Journal of Statistical Software, 63(8):1-25. doi:10.18637/jss.v063.i08.

Schulz, J., Jung, S., Huckemann, S., Pierrynowski, M., Marron, J. S., and Pizer, S. M. (2015). Analysis of rotational deformations from directional data. Journal of Computational and Graphical Statistics, 24(2):539-560. doi:10.1080/10618600.2014.914947.

Scott, J. G. (2011). Bayesian estimation of intensity surfaces on the sphere via needlet shrinkage and selection. Bayesian Analysis, 6(2):307-327. doi:10.1214/11-BA611.

Self, S. G. and Liang, K.-Y. (1987). Asymptotic properties of maximum likelihood estimators and likelihood ratio tests under nonstandard conditions. Journal of the American Statistical Association, 82(398):605-610. doi:10.1080/01621459.1987.10478472.

SenGupta, A. and Bhattacharya, S. (2015). Finite mixture-based Bayesian analysis of linearcircular models. Environmental and Ecological Statistics, 22(4):667-679. doi:10.1007/ s10651-015-0325-8.

SenGupta, A. and Laha, A. K. (2008). A likelihood integrated method for exploratory graphical analysis of change point problem with directional data. Communications in Statistics - Theory and Methods, 37(11-12):1783-1791. doi:10.1080/03610920701826401.

SenGupta, A. and Pal, C. (2001). On optimal tests for isotropy against the symmetric wrapped stable-circular uniform mixture family. Journal of Applied Statistics, 28(1):129-143. doi:10. 1080/02664760120011653.

SenGupta, A. and Roy, S. (2005). A simple classification rule for directional data. In Balakrishnan, N., Nagaraja, H. N., and Kannan, N. (Eds.), Advances in Ranking and Selection, Multiple Comparisons, and Reliability, Statistics for Industry and Technology, pp. 81-90. Birkhäuser, Boston. doi:10.1007/0-8176-4422-9_5.

SenGupta, A. and Ugwuowo, F. I. (2011). A classification method for directional data with application to the human skull. Communications in Statistics - Theory and Methods, 40(3):457-466. doi: $10.1080 / 03610920903377807$.

Shieh, G. S. and Johnson, R. A. (2005). Inference based on a bivariate distribution with von Mises marginals. Annals of the Institute of Statistical Mathematics, 57(4):789-802. doi:10.1007/ bf 02915439 .

Singh, H., Hnizdo, V., and Demchuk, E. (2002). Probabilistic model for two dependent circular variables. Biometrika, 89(3):719-723. doi:10.1093/biomet/89.3.719.

Sinz, F., Berens, B., Kuemmerer, M., and Wallis, T. (2018). PyCircStat: Circular Statistics with Python. URL: https://github.com/circstat/pycircstat.

Sittel, F., Filk, T., and Stock, G. (2017). Principal component analysis on a torus: theory and application to protein dynamics. The Journal of Chemical Physics, 147(24):244101. doi:10. 1063/1.4998259.

Sklar, M. (1959). Fonctions de répartition à $n$ dimensions et leurs marges. Publications de l'Institut de Statistique de l'Université de Paris, 8:229-231.

Small, C. G. (1987). Measures of centrality for multivariate and directional distributions. The Canadian Journal of Statistics, 15(1):31-39. doi:10.2307/3314859.

Soetaert, K. (2019). plot3D: Plotting Multi-Dimensional Data. R package version 1.3. URL: https: //CRAN.R-project. org/package=plot3D.

Sommer, S. (2013). Horizontal dimensionality reduction and iterated frame bundle development. In Nielsen, F. and Barbaresco, F. (Eds.), Geometric Science of Information, volume 8085 of Lecture Notes in Computer Science, pp. 76-83, Berlin. Springer. doi:10.1007/978-3-642-40020-9_7. 
Sommer, S. (2019). An infinitesimal probabilistic model for principal component analysis of manifold valued data. Sankhyā, Series A, 81(1):37-62. doi:10.1007/s13171-018-0139-5.

Sommer, S., Lauze, F., and Nielsen, M. (2014). Optimization over geodesics for exact principal geodesic analysis. Advances in Computational Mathematics, 40(2):283-313. doi:10.1007/ s10444-013-9308-1.

Souden, M., Kinoshita, K., and Nakatani, T. (2013). An integration of source location cues for speech clustering in distributed microphone arrays. In 2013 IEEE International Conference on Acoustics, Speech and Signal Processing, pp. 111-115, New York. IEEE. doi:10.1109/icassp. 2013.6637619.

Soukissian, T. H. (2014). Probabilistic modeling of directional and linear characteristics of wind and sea states. Ocean Engineering, 91:91-110. doi:10.1016/j.oceaneng.2014.08.018.

Sra, S. (2018). Directional statistics in machine learning: a brief review. In Ley, C. and Verdebout, T. (Eds.), Applied Directional Statistics, Chapman \& Hall/CRC Interdisciplinary Statistics Series, pp. 259-276. CRC Press, Boca Raton.

Sra, S. and Karp, D. (2013). The multivariate Watson distribution: maximum-likelihood estimation and other aspects. Journal of Multivariate Analysis, 114:256-269. doi:10.1016/j.jmva.2012. 08.010.

Stephens, M. A. (1982). Use of the von Mises distribution to analyse continuous proportions. Biometrika, 69(1):197-203. doi:10.1093/biomet/69.1.197.

Straub, J., Chang, J., Freifeld, O., and Fisher, J. W. I. (2015). A Dirichlet process mixture model for spherical data. In Lebanon, G. and Vishwanathan, S. V. N. (Eds.), Proceedings of the Eighteenth International Conference on Artificial Intelligence and Statistics, volume 38 of Proceedings of Machine Learning Research, San Diego. PMLR.

$\mathrm{Su}, \mathrm{Y}$. and $\mathrm{Wu}, \mathrm{X} .-\mathrm{K}$. (2011). Smooth test for uniformity on the surface of a unit sphere. In 2011 International Conference on Machine Learning and Cybernetics, pp. 867-872, New York. IEEE. doi:10.1109/icmlc.2011.6016757.

Sun, S. Z. and Lockhart, R. A. (2019). Bayesian optimality for Beran's class of tests of uniformity around the circle. Journal of Statistical Planning and Inference, 198:79-90. doi:10.1016/j. jspi.2018.03.006.

Taghia, J., Ma, Z., and Leijon, A. (2014). Bayesian estimation of the von-Mises Fisher mixture model with variational inference. IEEE Transactions on Pattern Analysis and Machine Intelligence, 36(9):1701-1715. doi:10.1109/tpami.2014.2306426.

Taijeron, H. J., Gibson, A. G., and Chandler, C. (1994). Spline interpolation and smoothing on hyperspheres. SIAM Journal on Scientific Computing, 15(5):1111-1125. doi:10.1137/0915068.

Takasu, Y., Yano, K., and Komaki, F. (2018). Scoring rules for statistical models on spheres. Statistics \& Probability Letters, 138:111-115. doi:10.1016/j.spl.2018.02.054.

Tang, H., Chu, S. M., and Huang, T. S. (2009). Generative model-based speaker clustering via mixture of von Mises-Fisher distributions. In 2009 IEEE International Conference on Acoustics, Speech and Signal Processing, pp. 4101-4104, New York. IEEE. doi:10.1109/icassp. 2009. 4960530.

Taniguchi, M., Kato, S., Ogata, H., and Pewsey, A. (2020). Models for circular data from time series spectra. Journal of Time Series Analysis, to appear. doi:10.1111/jtsa.12549.

Taylor, C. C. (2008). Automatic bandwidth selection for circular density estimation. Computational Statistics \& Data Analysis, 52(7):3493-3500. doi:10.1016/j.csda.2007.11.003.

Taylor, C. C., Lafratta, G., and Fensore, S. (2018). nprotreg: Nonparametric Rotations for SphereSphere Regression. R package version 1.0.1. URL: https://CRAN.R-project.org/package= nprotreg.

Tipping, M. E. and Bishop, C. M. (1999). Probabilistic principal component analysis. Journal of the Royal Statistical Society, Series B (Statistical Methodology), 61(3):611-622. doi:10.1111/ 1467-9868.00196.

Traa, J. and Smaragdis, P. (2013). A wrapped Kalman filter for azimuthal speaker tracking. IEEE 
Signal Processing Letters, 20(12):1257-1260. doi:10.1109/1sp.2013.2287125.

Tsagris, M. and Alenazi, A. (2019). Comparison of discriminant analysis methods on the sphere. Communications in Statistics: Case Studies, Data Analysis and Applications, 5(4):467-491. doi: 10.1080/23737484.2019.1684854.

Tsagris, M., Athineou, G., Sajib, A., Amson, E., and Waldstein, M. J. (2020). Directional: Directional Statistics. R package version 4.4. URL: https://CRAN.R-project.org/package= Directional.

Tsai, M.-T. (2009). Asymptotically efficient two-sample rank tests for modal directions on spheres. Journal of Multivariate Analysis, 100:445-458. doi:10.1016/j.jmva.2008.05.009.

Tsuruta, Y. and Sagae, M. (2017a). Asymptotic property of wrapped Cauchy kernel density estimation on the circle. Bulletin of Informatics and Cybernetics, 49:1-10. doi:10.5109/2232318.

Tsuruta, Y. and Sagae, M. (2017b). Higher order kernel density estimation on the circle. Statistics \& Probability Letters, 131:46-50. doi:10.1016/j .spl.2017.08.003.

Tsuruta, Y. and Sagae, M. (2018). Properties for circular nonparametric regressions by von Miese and wrapped Cauchy kernels. Bulletin of Informatics and Cybernetics, 50:1-13.

Tsuruta, Y. and Sagae, M. (2020). Theoretical properties of bandwidth selectors for kernel density estimation on the circle. Annals of the Institute of Statistical Mathematics, 72(2):511-530. doi: $10.1007 / \mathrm{s} 10463-018-0701-\mathrm{x}$.

Tung, D. D. and Jammalamadaka, S. R. (2013). On the Gini mean difference test for circular data. Communications in Statistics - Theory and Methods, 42(11):1998-2008. doi:10.1080/03610926. 2011.601947.

Umbach, D. and Jammalamadaka, S. R. (2009). Building asymmetry into circular distributions. Statistics \&5 Probability Letters, 79(5):659-663. doi:10.1016/j.spl.2008.10.022.

van der Vaart, A. W. (2000). Asymptotic Statistics. Cambridge Series in Statistical and Probabilistic Mathematics. Cambridge University Press, Cambridge. doi:10.1017/CB09780511802256.

Veeraraghavan, A., Srivastava, A., Roy-Chowdhury, A. K., and Chellappa, R. (2009). Rate-invariant recognition of humans and their activities. IEEE Transactions on Image Processing, 18(6):13261339. doi:10.1109/TIP.2009.2017143.

Verdebout, T. (2015). On some validity-robust tests for the homogeneity of concentrations on spheres. Journal of Nonparametric Statistics, 27(3):372-383. doi:10.1080/10485252.2015. 1041945.

Verdebout, T. (2017). On the efficiency of some rank-based test for the homogeneity of concentrations. Journal of Statistical Planning and Inference, 191:101-109. doi:10.1016/j.jspi.2017. 05.009.

Vuollo, V. and Holmstrom, L. (2018). A scale space approach for exploring structure in spherical data. Computational Statistics \& Data Analysis, 125:57-69. doi:10.1016/j.csda.2018.03.014.

Vuollo, V., Holmström, L., Aarnivala, H., Harila, V., Heikkinen, T., Pirttiniemi, P., and Valkama, A. M. (2016). Analyzing infant head flatness and asymmetry using kernel density estimation of directional surface data from a craniofacial 3D model. Statistics in Medicine, 35(26):4891-4904. doi: $10.1002 / \mathrm{sim} .7032$.

Wang, F. (2013). Space and Space-Time Modeling of Directional Data. PhD thesis, Duke University.

Wang, F. and Gelfand, A. E. (2013). Directional data analysis under the general projected normal distribution. Statistical Methodology, 10(1):113-127. doi:10.1016/j.stamet.2012.07.005.

Wang, F. and Gelfand, A. E. (2014). Modeling space and space-time directional data using projected Gaussian processes. Journal of the American Statistical Association, 109(508):1565-1580. doi: 10.1080/01621459.2014.934454.

Wang, F., Gelfand, A. E., and Jona-Lasinio, G. (2015). Joint spatio-temporal analysis of a linear and a directional variable: space-time modeling of wave heights and wave directions in the Adriatic Sea. Statistica Sinica, 25(1):25-39.

Wang, J., Boyer, J., and Genton, M. G. (2004). A skew-symmetric representation of multivariate distributions. Statistica Sinica, 14(4):1259-1270. 
Wang, M. and Shimizu, K. (2012). On applying Möbius transformation to cardioid random variables. Statistical Methodology, 9(6):604-614. doi:10.1016/j.stamet.2012.04.001.

Wang, M. and Wang, D. (2016). VMF-SNE: embedding for spherical data. In 2016 IEEE International Conference on Acoustics, Speech and Signal Processing (ICASSP), pp. 2344-2348, New York. IEEE. doi:10.1109/icassp.2016.7472096.

Wang, X. and Zhao, L. (2001). Laws of the iterated logarithm for kernel estimator of density function of spherical data. Journal of Systems Science and Mathematical Sciences, 21(3):264-273.

Wang, X., Zhao, L., and Wu, Y. (2000). Distribution free laws of the iterated logarithm for kernel estimator of regression function based on directional data. Chinese Annals of Mathematics. Series $B, 21(4): 489-498$. doi:10.1142/S0252959900000480.

Wang, X. M. (2002). Exponential bounds of mean error for the kernel regression estimates with directional data. Chinese Annals of Mathematics. Series A, 23(1):55-62.

Wang, X. M. and Ma, L. (2000). Nearest neighbor estimator for density function of directional data. Journal of Biomathematics, 15(3):332-338.

Wang, X. M. and Zhao, L. C. (2003). A law of logarithm for kernel density estimators with directional data. Acta Mathematica Sinica, Chinese Series, 46(5):865-874.

Watamori, Y. and Jupp, P. E. (2005). Improved likelihood ratio and score tests on concentration parameters of von Mises-Fisher distributions. Statistics 83 Probability Letters, 72(2):93-102. doi: 10.1016/j.spl.2004.10.017.

Watson, G. S. (1961). Goodness-of-fit tests on a circle. Biometrika, 48(1/2):109-114. doi:10.2307/ 2333135.

Watson, G. S. (1983). Statistics on Spheres. University of Arkansas Lecture Notes in the Mathematical Sciences. Wiley, New York.

Wehrly, T. E. and Johnson, R. A. (1980). Bivariate models for dependence of angular observations and a related Markov process. Biometrika, 67(1):255-256. doi:10.1093/biomet/67.1.255.

Wilson, R. C., Hancock, E. R., Pekalska, E., and Duin, R. P. W. (2014). Spherical and hyperbolic embeddings of data. IEEE Transactions on Pattern Analysis and Machine Intelligence, 36(11):2255-2269. doi:10.1109/tpami.2014.2316836.

Wood, S. N. (2017). Generalized Additive Models. Chapman \& Hall/CRC Texts in Statistical Science Series. CRC Press, Boca Raton, second edition. doi:10.1201/9781315370279.

Wouters, H., Thas, O., and Ottoy, J.-P. (2009). Data-driven smooth tests and a diagnostic tool for lack-of-fit for circular data. Australian $\&$ New Zealand Journal of Statistics, 51(4):461-480. doi:10.1111/j.1467-842X.2009.00558.x.

$\mathrm{Xu}$, D. and Wang, Y. (2020). Area-proportional visualization for circular data. Journal of Computational and Graphical Statistics, 29(2):351-357. doi:10.1080/10618600.2019.1654881.

Yamaji, A. and Sato, K. (2011). Clustering of fracture orientations using a mixed Bingham distribution and its application to paleostress analysis from dike or vein orientations. Journal of Structural Geology, 33(7):1148-1157. doi:10.1016/j.jsg.2011.05.006.

Yang, M.-S., Chang-Chien, S.-J., and Kuo, H.-C. (2014). On mean shift clustering for directional data on a hypersphere. In Rutkowski, L., Korytkowski, M., Scherer, R., Tadeusiewicz, R., Zadeh, L. A., and Zurada, J. M. (Eds.), Artificial Intelligence and Soft Computing, volume 8468 of Lecture Notes in Compututer Scence, pp. 809-818, Cham. Springer. doi:10.1007/978-3-319-07176-3_70.

Yang, M.-S. and Pan, J.-A. (1997). On fuzzy clustering of directional data. Fuzzy Sets and Systems, 91(3):319-326. doi:10.1016/s0165-0114(96)00157-1.

Yeh, S.-Y., Harris, K. D. M., and Jupp, P. E. (2013). A drifting Markov process on the circle, with physical applications. Proceedings of the Royal Society A: Mathematical, Physical and Engineering Sciences, 469(2156):20130092. doi:10.1098/rspa.2013.0092.

You, K. (2020). RiemBase: Functions and $C++$ Header Files for Computation on Manifolds. R package version 0.2.4. URL: https://CRAN.R-project.org/package=RiemBase.

Zhan, X., Ma, T., Liu, S., and Shimizu, K. (2019). On circular correlation for data on the torus. Statistical Papers, 60(6):1827-1847. doi:10.1007/s00362-017-0897-5. 
Zhang, L., Li, Q., Guo, Y., Yang, Z., and Zhang, L. (2018). An investigation of wind direction and speed in a featured wind farm using joint probability distribution methods. Sustainability, 10(12):4338. doi:10.3390/su10124338.

Zhang, M. and Fletcher, T. (2013). Probabilistic principal geodesic analysis. In Burges, C. J. C., Bottou, L., Welling, M., Ghahramani, Z., and Weinberger, K. Q. (Eds.), Advances in Neural Information Processing Systems 26, pp. 1178-1186, Red Hook. Curran Associates.

Zhang, Z., Klassen, E., and Srivastava, A. (2019). Robust comparison of kernel densities on spherical domains. Sankhyā, Series A, 81(1):144-171. doi:10.1007/s13171-018-0131-0.

Zhao, L. and Wu, C. (2001). Central limit theorem for integrated squared error of kernel estimators of spherical density. Science in China Series A: Mathematics, 44(4):474-483. doi:10.1007/ bf 02881884 .

Zou, G., Hua, J., and Muzik, O. (2007). Non-rigid surface registration using spherical thin-plate splines. In Ayache, N., Ourselin, S., and Maeder, A. (Eds.), Medical Image Computing and Computer-Assisted Intervention - MICCAI 200\%, pp. 367-374, Berlin. Springer. doi:10.1007/ 978-3-540-75757-3_45.

Zucchini, W., MacDonald, I. L., and Langrock, R. (2016). Hidden Markov Models for Time Series, volume 150 of Monographs on Statistics and Applied Probability. CRC Press, Boca Raton, second edition. doi:10.1201/b20790. 